\title{
A cell-type and compartment specific analysis of glutathione and hydrogen peroxide
}

\author{
Dissertation \\ for the award of the degree \\ "Doctor rerum naturalium" \\ of the Georg-August-Universität Göttingen \\ within the doctoral program Molecular Medicine \\ of the Georg-August University School of Science (GAUSS)
}

submitted by

Irina Karoline Trautsch, née Eckhardt

(born in Göttingen, Germany)

Göttingen, April 2019 



\section{Examination board members}

Prof. Wolfram-Hubertus Zimmermann (Supervisor, $1^{\text {st }}$ Referee)

Institute of Pharmacology and Toxicology, University Medical Center Göttingen

Prof. Henning Urlaub (Thesis Advisory Committee Member, $2^{\text {nd }}$ Referee)

Bioanalytical Mass Spectrometry, MPI for Biophysical Chemistry, Göttingen

Dr. Katrin Streckfuss-Bömecke (Thesis Advisory Committee Member)

Translational Stem Cell Research, Clinic for Cardiology and Pneumology, University Medical Center Göttingen

Prof. Matthias Dobbelstein

Institute of Molecular Oncology, University Medical Center Göttingen

Prof. Ralf Dressel

Institute of Cellular and Molecular Immunology, University Medical Center Göttingen

Prof. Thomas Meyer

Molecular Psychocardiology, Clinic for Psychosomatic Medicine and Psychotherapy, University Medical Center Göttingen

Prof. Manuel Mayr (IRTG1816 Co-Supervisor)

Cardiovascular Proteomics, British Heart Foundation Centre of Research Excellence, King's College London

Date of disputation: 19.06.2019 


\section{Acknowledgements}

I would like to express my deepest gratitude to everyone who supported me and this thesis during the past years.

First, I'd like to thank Prof Zimmermann for giving me the opportunity to work in his institute. I appreciate the freedom and trust that I was given to shape and pursue this project within the IRTG1816 and the helpful advice and support.

Furthermore, I would like to thank my thesis committee members, Prof Henning Urlaub and Dr. Katrin Streckfuss-Boemecke, who gave valuable advice and offered uncomplicated help when needed. My thanks also extend to my examination board members, Prof. Matthias Dobbelstein, Prof. Ralf Dressel and Prof. Thomas Meyer for taking their time to evaluate this project. I would like to further thank my IRTG 1816 co-supervisor Prof. Manual Mayr for his support and patience while watching the project develop.

A big shout-out goes to Dr. PL Soong who agreed to co-supervise this thesis. He took great care (sometimes from a distance) that I don't lose time and focus and get my work done the right way. Thank you for your input and for sharing your extensive knowledge, not only on stem cell biology, with me. I learned a lot.

Further I'm especially grateful for the kind support and input I received from outside our institute. Prof. Seva Belousov and Prof. Ivan Bogeski provided me with ideas, plasmids and feedback that helped me to set-up and pursue my project. I thank the whole Bogeski lab, especially Xin, for not only allowing me to use their imaging set-up, but also teaching me how to use it correctly and making bookings possible even in times of high use.

A lot of the work I present in this thesis would not have been possible without the support and help of many (current and former) colleagues in the institute. I especially thank Franzi, Monique, Lavanya Denise and Eric for their help with molecular biology; PL and Eriona for taking over lentiviral experiments when S2 regulations kicked in; Susanne S., Norman and Sebastian for the occasional generation and maintenance of EHMs; Patapia for sharing her knowledge and experience with TALENs and AAVS1; the human lab team for media preparation, stem cell culture advice, countless differentiation runs and much more; my office mates Mina, Franzi, Elif, Maja and formerly Shu for their support and help and for bearing with my need to complain from time to time. A big thanks also goes to all my business travel mates, especially Norman, Farah, Patapia and PL for a memorable ISSCR conference. 
I especially appreciate the work undertaken by Susanne S., Franzi and Monique as well as my fellow IRTG colleagues Lisa, Mausi and by extension non-elite Eric to ease the strain of PhD times by offering their time and emotional support. I hope I could help you keep your sanity as well.

Another shout-out goes to my fellow MolMeds, in and outside of Göttingen. We started this journey together and I'm grateful that we still keep in touch.

I thank my family for always being a safe harbor when I needed it and supporting me in any possible way. I feel very priviledged to have such strong roots. The last and biggest thanks go out to Fabian. You might be a support main, but to me you were the hyper carry in this game. 



\section{Table of Contents}

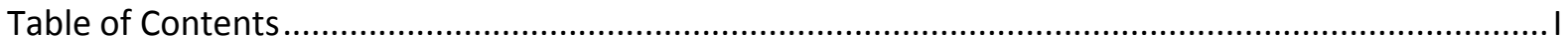

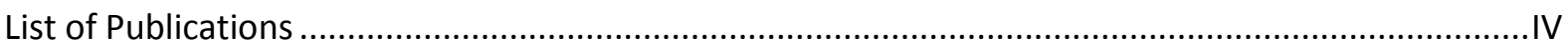

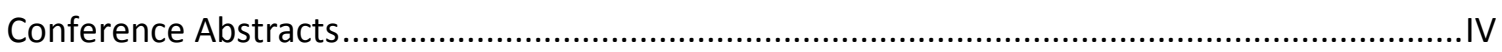

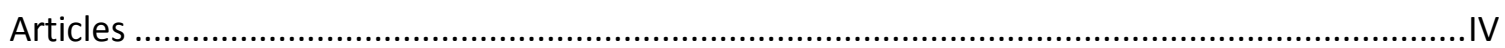

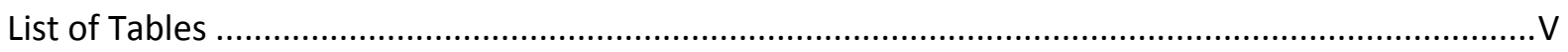

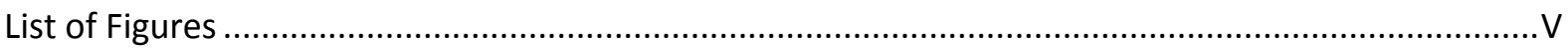

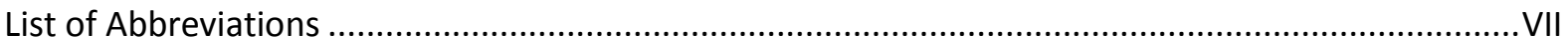

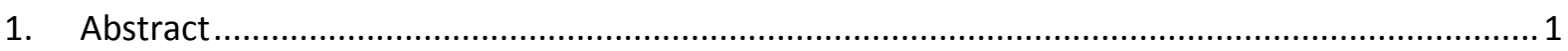

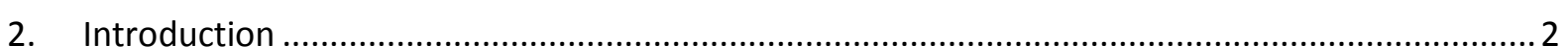

2.1. Reactive Oxygen Species and their role in the cardiovascular system ................................

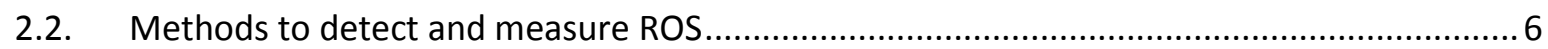

2.3. Stem cells and engineered tissues as model systems....................................................11

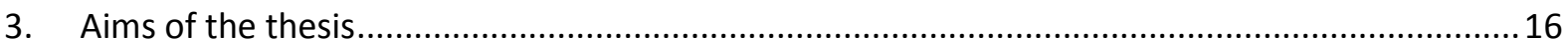

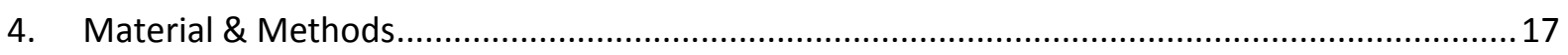

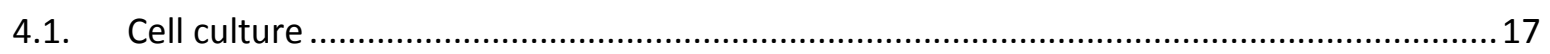

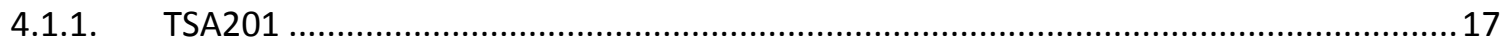

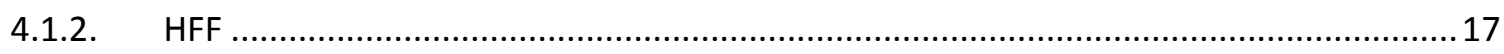

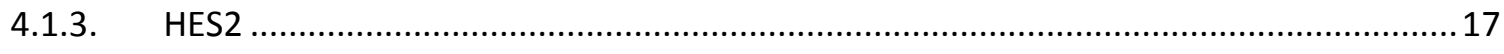

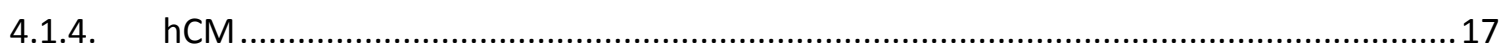

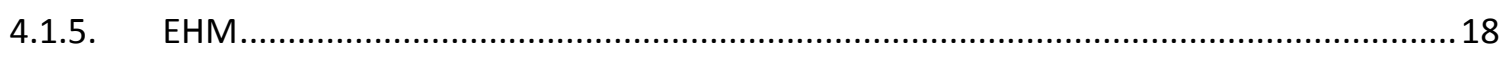

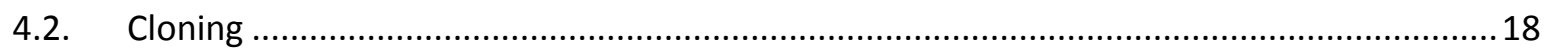

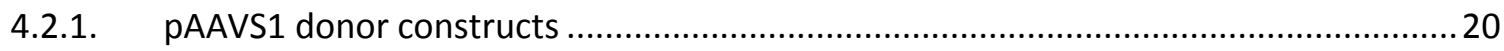

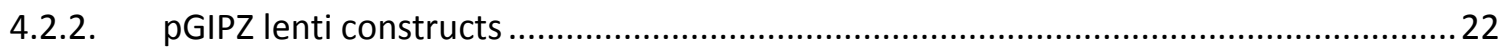

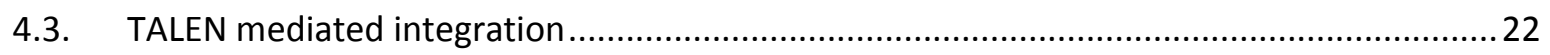

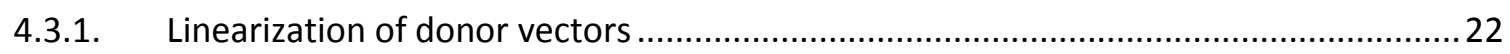

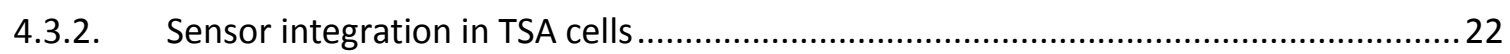

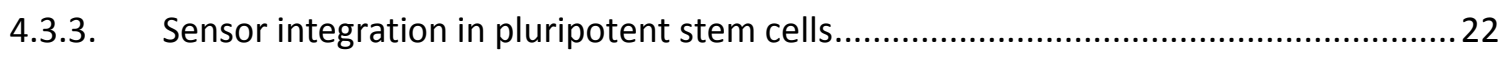

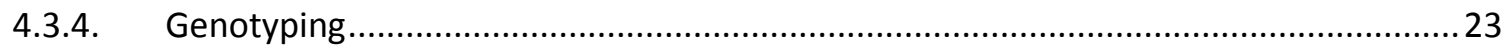

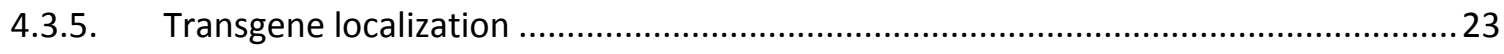




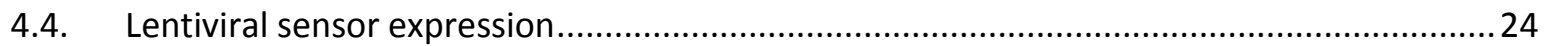

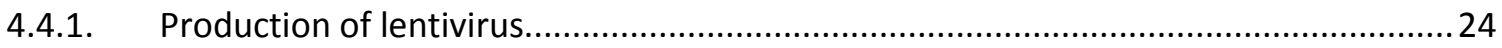

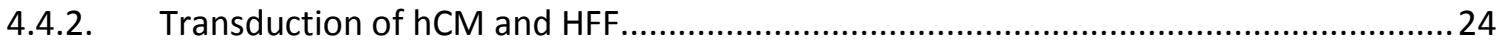

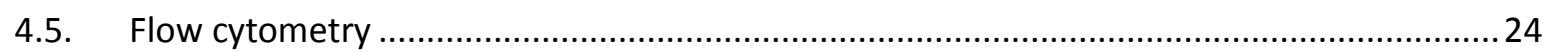

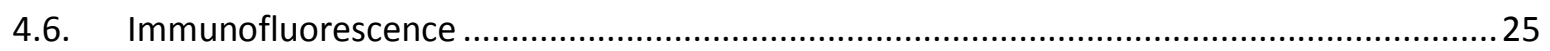

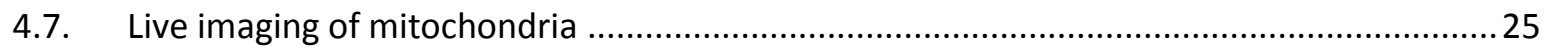

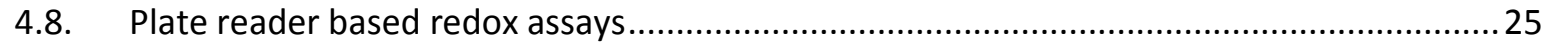

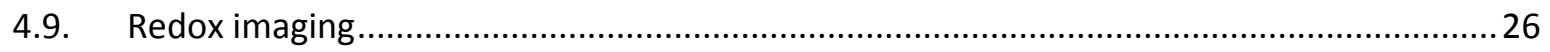

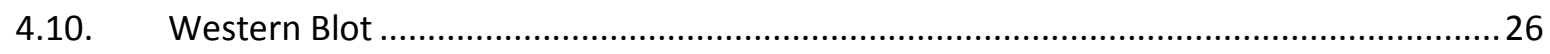

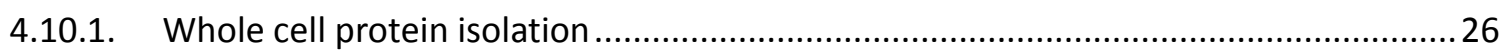

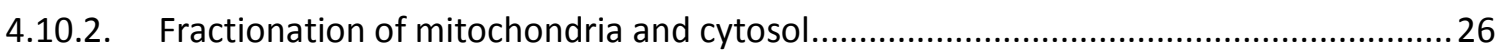

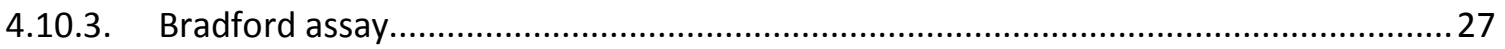

4.10.4. SDS-polyacrylamid gel electrophoresis and western blot ........................................2

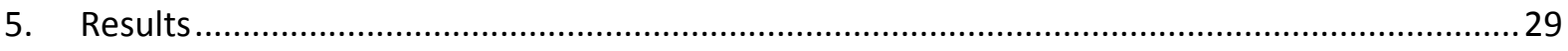

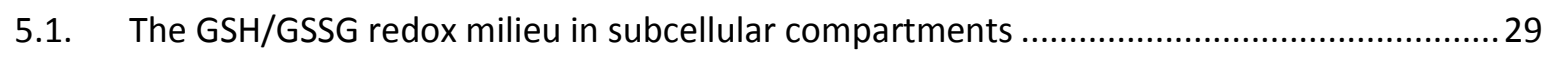

5.1.1. Grx1-roGFP2 can be targeted to specific compartments in TSA cells .........................29

5.1.2. Plate reader based redox assays confirm function of Grx1-roGFP2 in TSA cells ..........30

5.1.3. Genomic integration leads to redox sensor expression in HES2 ................................ 31

5.1.4. Undifferentiated HES2 show a more reduced cytosol compared to mitochondria ......34

5.1.5. HES2cyto\#3 and HES2 mito2\#34 derived cardiomyocytes are developmentally impaired 36

5.1.6. Stem cell derived cardiomyocytes have more oxidized mitochondria .........................38

5.1.7. Redox imaging of engineered heart muscle ........................................................... 38

5.1.8. Lentivirus mediated expression of mito-Grx1-roGFP2 ............................................. 41

5.2. Sensing and producing $\mathrm{H}_{2} \mathrm{O}_{2}$ in cell-cell interaction models .........................................46

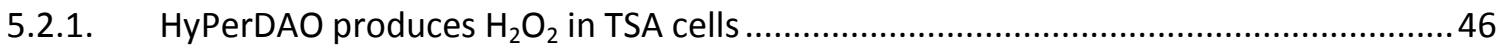

5.2.2. HES2 integrate and express HyPer expression sequences .........................................49

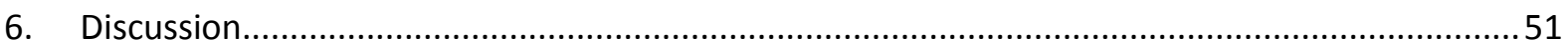




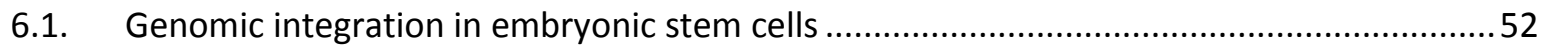

6.2. Cardiac differentiation is deficient in transgenic HES lines ............................................53

6.3. The GSH/GSSG redox balance in cardiomyocyte mitochondria ........................................55

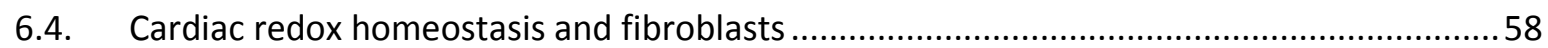

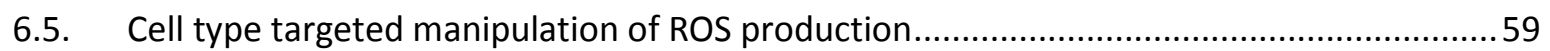

6.6. Applicability and use of fluorescent redox sensors in redox research ..............................60

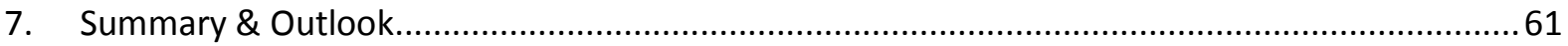

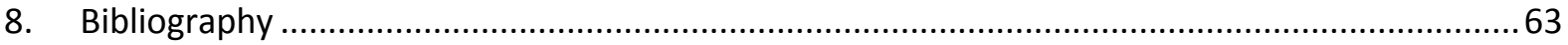

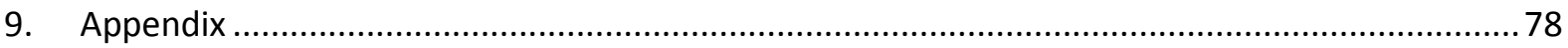

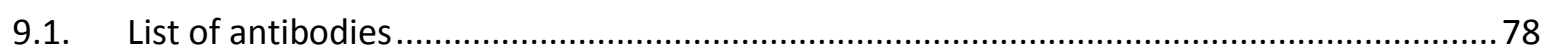

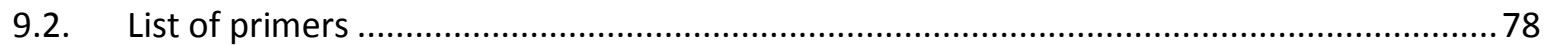

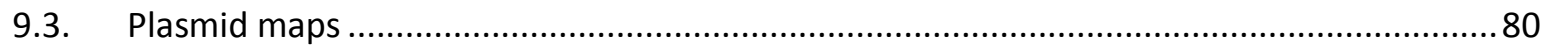




\section{List of Publications}

\section{Conference Abstracts}

Eckhardt, Irina, Soong, Poh Loong, Mayr, Manuel, \& Zimmermann, Wolfram-Hubertus. (2017). Redox Regulation of Human Engineered Heart Muscle Function. Presented at the BHF Postgraduate Symposium 2017, London, UK.

Eckhardt, Irina, Heta, Eriona, Soong, Poh Loong, \& Zimmermann, Wolfram-Hubertus. (2017). Redox Regulation of Human Engineered Heart Muscle Function. Presented at the DGK Herztage - Basic Science Meeting, Berlin, GER.

Eckhardt, Irina, Soong, P. L., Belousov, Vsevolod V, Mayr, Manuel, \& Zimmermann, WolframHubertus. (2018). Redox live: sensing the redox state of human stem cell derived cardiomyocytes. Presented at the JCl International Symposium - Advances in Heart Failure, London, UK.

Eckhardt, Irina, Soong, Poh Loong, Belousov, Vsevolod V, \& Zimmermann, Wolfram-Hubertus. (2018). GSH and H2O2 redox assessment in human stem cell derived cardiomyocytes. Presented at the 3rd German Pharm-Tox Summit, Göttingen, GER.

Eckhardt, Irina, Soong, Poh Loong, Mayr, Manuel, \& Zimmermann, Wolfram-Hubertus. (2018). Redox Live: Gluthathione Redox Potential Sensing in Cellular Compartments. Presented at the BHF Postgraduate Symposium 2018, London, UK.

Eckhardt, Irina, Soong, Poh Loong, \& Zimmermann, Wolfram-Hubertus. (2018). Redox Live: Gluthathione Redox Potential Sensing in Cellular Compartments. Presented at the ISSCR 2018 Annual Meeting, Melbourne, AUS.

\section{Articles}

Trautsch, I., Heta, E., Soong, P. L., Levent, E., Nikolaev, V. O., Bogeski, I., Katschinski, D. M., Mayr, M., \& Zimmermann, W.-H. (2019). Optogenetic Monitoring of the Glutathione Redox State in Engineered Human Myocardium. Frontiers in Physiology, 10. 


\section{List of Tables}

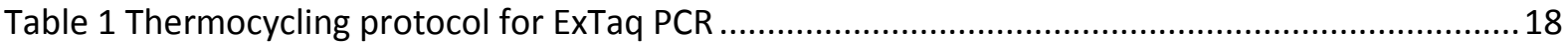

Table 2 Thermocycling protocol for PrimeStar-HS PCR …..............................................................18

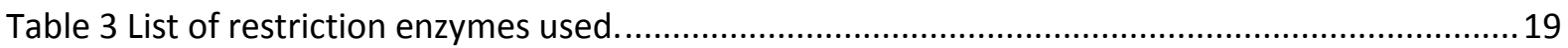

Table 4 Thermocycling protocol for genotyping and colony PCR with FastGene polymerase .............23

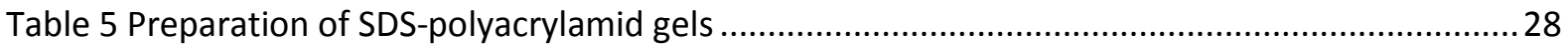

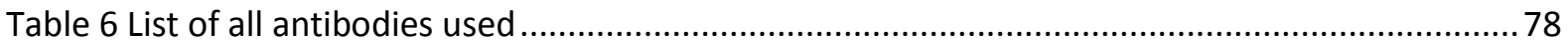

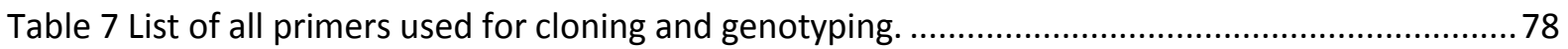

\section{List of Figures}

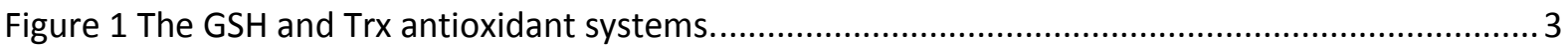

Figure 2 Basic principle of fluorescent protein redox sensors........................................................... 9

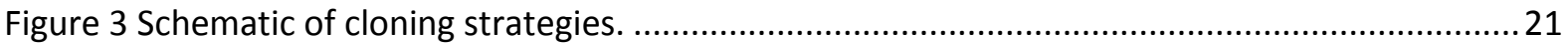

Figure 4 Grx1-roGFP2 is expressed in a compartment specific manner in TSA cells...........................30

Figure 5 Grx1-roGFP2 expressed in cytosol or mitochondria of TSA cells reacts to oxidation and

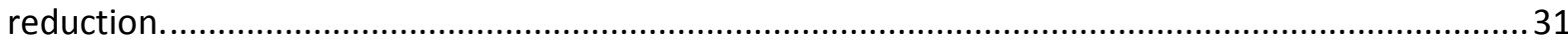

Figure 6 Transgene integration does not influence pluripotency. .................................................32

Figure 7 Redox sensor transgenes integrated at random sites in the HES2 genome..........................33

Figure 8 Grx1-roGFP is expressed in the targeted subcellular compartments in transgenic HES2 .....34

Figure 9 HES Grx1-roGFP2 and mito-Grx1-roGFP2 react to oxidation and reduction in a concentration

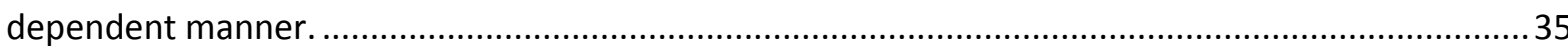

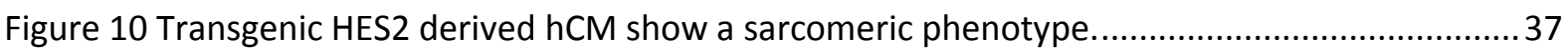

Figure 11 Redox sensors are functional in stem cell derived cardiomyocytes...................................38

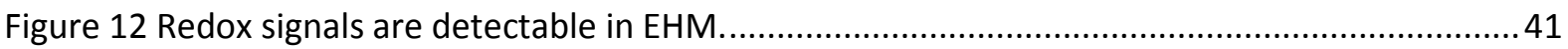

Figure 13 Transduced HFF report oxidation and reduction in cytosol and mitochondria....................43

Figure 14 Transduced hCM mitochondria are oxidized compared to cytosol....................................45

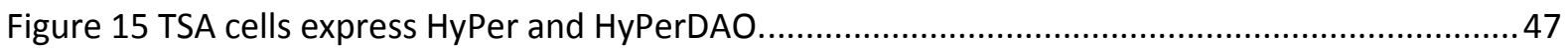

Figure 16 HyPer and HyPerDAO is functional in TSA cells. .................................................................. 48

Figure 17 HyPer expression in HES2 does not influence pluripotency...............................................49

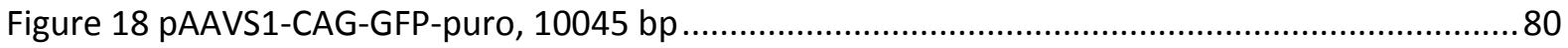

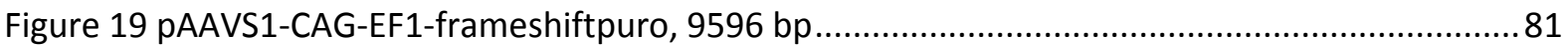

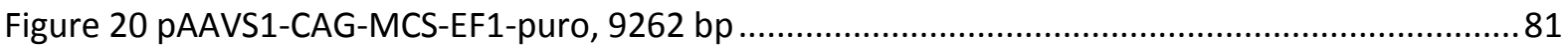

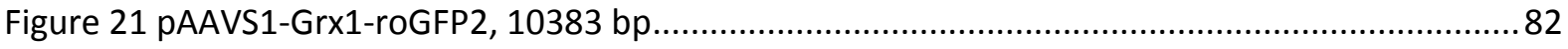

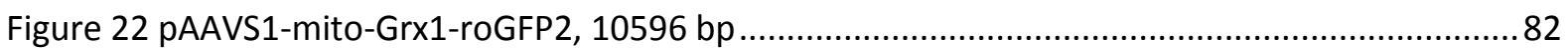




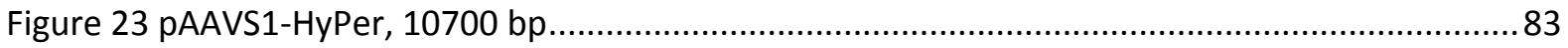

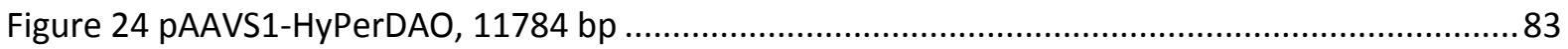

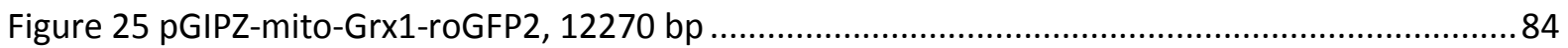




\section{List of Abbreviations}

$2-\mathrm{OH}-\mathrm{E}^{+}$

AAV9

$\alpha \mathrm{MHC}$

Ang 2

APS

ATP

$\mathrm{BH} 4$

BMP

BSA

CAG

CamKII

CMV

CRISPR

DNA

DHE

DTT

EDTA

EF1

eGFP

$\mathrm{E}_{\mathrm{GSH}}$

EHM

FCS

FGF2

FSC

GAPDH

Gpx

Grx

GSH

GSSG

H2DCF-DA

$\mathrm{H}_{2} \mathrm{O}_{2}$

hCM

HEPES
2-Hydroxyethidium adeno-associated virus serotype 9 a myosin heavy chain angiotensin 2 ammonium persulfate adenosintriphosphate tetrahydrobiopterin bone morphogenetic protein bovine serum albumin CMV early enhancer chicken beta actin rabbit beta globin Calcium-calmodulin dependent kinase II cytomegalovirus clustered regularly interspaced short palindromic repeats desoxyribonucleic acid dihydroethidium dithiotreithol ethylenediaminetetraacetic acid elongation factor 1 enhanced green fluorescent protein GSH redox potential engineered heart muscle fetal calf serum fibroblast growth factor 2 forward scatter glycerolaldehydephosphatedehydrogenase gluthathionperoxidase glutharedoxin gluthathion (reduced) gluthathion (oxidized) dichlorodihydrofluorescein diacteate hydrogen peroxide human cardiomyocyte 4-(2-hydroxyethyl)-1-piperazineethanesulfonic acid 
HES

HFF

Hif $1 \alpha$

hpf

HRP

iPSC

IWP

KIf2

LB

MAO

MCS

mPTP

MsrA

$\mathrm{NADPH}$

NF-kappaB

NO

NOX

Oct4

ORF

PBS

PCR

PFA

PGK

PKA

Prx

PVDF

RNA

ROCK

ROI

ROS

RPMI

RVD

RyR2

SDS human embryonic stem cells human foreskin fibroblast hypoxia inducible factor $1 a$ hours post fertilization horse raddish peroxidase induced pluripotent stem cell ihibitor of wnt production krüppel-like factor 2 lysogeny broth mono-amino oxidase multiple cloning site mitchondria permeability transition pore methionine sulfoxide reductase $A$ nicotinamide adenine dinucleotide phosphate nuclear factor kappa-light-chain-enhancer of activated B cells nitric oxide NADPH-oxidase octamer binding transcription factor 4 open reading frame phosphate buffered saline polymerase chain reaction paraformaldehyde phospho glycerate kinase protein kinase $A$ peroxiredoxin polyvinylidene difluoride ribonucleic acid rho-associated protein kinase region of interest reactive oxygen species Roswell Parks Memorial Institute repeat-variable di-residue ryanodine receptor 2 sodium dodecyl sulfate 
SERCA

Sox2

SSC

TALEN

TBS-T

TEMED

TMRM

Tris

$\operatorname{Trx}$

TrxR

XO

YFP sarco/endoplasmic reticulum calcium ATPase sex determining region y-box 2 sideward scatter transcription-activator like effector nuclease Tris-buffered saline with tween tetramethylethylenediamine tetramethylrhodamine methylester tris(hydroxymethyl)aminomethane thioredoxin thioredoxin reductase xhantine oxidase yellow fluorescent protein 


\section{Abstract}

Reactive oxygen species (ROS) have been recognized to play important roles as messenger molecules. They are involved in the etiology and progression of cardiovascular diseases such as myocardial infarction, cardiac hypertrophy or fibrosis. Investigation of reactive oxygen species and cellular redox alterations has been methodologically challenging. Genetically encoded redox sensors make dynamic measurements of ROS and involved buffering systems in living cells possible. They can be targeted to specific cellular organelles and report compartment specific redox changes. Analysis of sensor response is performed by dual excitation single emission fluorescence microscopy or plate reader based assays. In the present study, we hypothesized that fluorescent redox sensors can be applied in human pluripotent stem cells and stem cell derived cardiomyocytes to investigate cell type and compartment specific differences in redox homeostasis. Genomic integration of two redox sensor systems was performed in human embryonic stem cells. Grx1-roGFP2 reacts and stays in equilibrium with cellular glutathione (GSH) pools, while HyPer can report increases in cellular hydrogen peroxide $\left(\mathrm{H}_{2} \mathrm{O}_{2}\right)$. Grx1-roGFP2 was targeted to cytosol and mitochondria in transgenic stem cells to analyze their GSH balance in the two compartments. Stem cell mitochondria appeared to have a more oxidized GSH pool than the cytosol. Transgenic stem cell lines were also differentiated to cardiomyocytes, but failed to develop spontaneous beating activity. Due to this unexpected phenotype, a lentiviral integration approach was chosen to express Grx1-roGFP2 in cytosol and mitochondria of stem cell derived cardiomyocytes and primary dermal fibroblasts. While fibroblast GSH milieu appeared similar between cytosol and mitochondria, cardiomyocytes had strongly oxidized mitochondria compared to cytosol. As reactive oxygen species can be generated during oxidative phosphorylation in mitochondria, cellular metabolism might play an important role in the regulation of cellular redox homoeostasis. As a model of advanced maturation, stem cell derived cardiomyocytes were used to construct engineered heart muscle. Preliminary proof-of-concept for the analysis of redox sensors in this tissue model is reported in this study. To sense $\mathrm{H}_{2} \mathrm{O}_{2}$, we employed an alternative sensor, namely HyPer, which was able to report changes in intracellular $\mathrm{H}_{2} \mathrm{O}_{2}$ levels upon extracellular bolus addition of $\mathrm{H}_{2} \mathrm{O}_{2}$. Aside from detection of $\mathrm{H}_{2} \mathrm{O}_{2}$, also a sensorproducer hybrid construct, HyperDAO, was deployed in a TSA model. Upon stimulation with Dalanine, HyPerDAO produced $\mathrm{H}_{2} \mathrm{O}_{2}$ inside transgenic cells. Unfortunately, human embryonic stem cells transgenic for HyPer or HyPerDAO also failed to differentiate into beating cardiomyocytes. In summary, in this thesis tools for the analysis and manipulation of ROS and their buffering systems in a cell- and compartment-specific manner were developed. Further studies are needed to elucidate the mechanisms behind differences in cellular redox balances reported here and will lead to a deeper understanding of redox signaling in cardiac physiology and pathology. 


\section{Introduction}

\subsection{Reactive Oxygen Species and their role in the cardiovascular system}

Reactive Oxygen species (ROS) are characterized by free electron pairs and high reactivity. The superoxide anion, $\mathrm{O}_{2}{ }^{-}$, is derived from the reduction of molecular oxygen. Inside cells, it rapidly reacts with water to form hydrogen peroxide $\left(\mathrm{H}_{2} \mathrm{O}_{2}\right)$, a strong and more stable oxidant. $\mathrm{H}_{2} \mathrm{O}_{2}$ interacts with reactive protein residues and thereby alters overall protein conformation, function and stability. Among the possible modifications, the oxidation of cysteines is best studied. Accessible thiol groups are oxidized to form a sulfenic acid. If sulfenic acid is in proximity to another cysteine residue, an intra- or intermolecular disulfide can be formed, a very common form of protein activity regulation. It has been described for example that disulfide formation in the catalytic subunit of protein kinase A (PKA) can lead to protein dephosphorylation and kinase inactivation (Humphries et al., 2005). Disulfides can be reduced by cellular reductants such as the gluthathione (GSH) or thioredoxin (Trx) systems. Sulfenic acid can also form adducts with GSH leading to Sglutathionylation of the target protein, another activity modulating post translational modification (Shackelford et al., 2005). If reactive nitrogen species such as nitric oxide (NO) are present, Snitrosylation of the sulfenic residue is also possible. Besides modulating protein activity as described for histone deacetylase 2 and PKA (Burgoyne \& Eaton, 2009; Nott et al., 2008), nitrosylation might also be a protective mechanism preventing further oxidation of the thiol group. If sulfenic acid is oxidized further, sulfinic acid is formed. This modification can be reduced by sulfiredoxin (Biteau et al., 2003). Further oxidation yields sulfonic acid, a modification that is thought to be irreversible and is associated with protein inhibition by destruction of catalytic thiol groups. Similarly irreversible is the addition of carbonyl groups, including ketones and aldehydes that result from lipid peroxidation. Protein carbonylation is considered to lead to accumulation and enhanced degradation of targeted proteins (Wong et al., 2010). Possible targets of protein carbonylation include cysteine, lysine, arginine, proline and threonine.

Other oxidized amino acids include methionine, tyrosine and tryptophan. Methionine oxidation yields a methionine sulfoxide that can be reduced through methionine sulfoxide reductase (MsrA) and plays a role in signaling, ROS scavenging, enzyme activity modulation and can target proteins for degradation (Stadtman et al., 2003). Activity modulation through methionine oxidation has been described for calcium-calmodulin dependent kinase II (CamKII), where an angiotensin 2 (Ang 2) induced oxidation of Met281/282 leads to kinase activation. This process might be relevant in cardiac pathology, as MsrA knock out mice show a more severe cardiac dysfunction with elevated CamKII oxidation after myocardial infarction (Erickson et al., 2008). Regarding tyrosine and 
tryptophan oxidation, only little is known. Tyrosine forms dityronsines upon reaction with oxidants such as $\mathrm{H}_{2} \mathrm{O}_{2}$, whereas Tryptophan is mostly a target for nitration (Shao et al., 2012).

In order to limit the accumulation and spread of oxidants as well as oxidative modifications, cells possess several defense mechanisms. Superoxide is detoxified by superoxide dismutates, which catalyze the reaction with water to $\mathrm{H}_{2} \mathrm{O}_{2}$. In turn, $\mathrm{H}_{2} \mathrm{O}_{2}$ can be degraded by catalases to water. The major cellular defense line against oxidative stress, however, is the glutathione system. Glutathionperoxidase (Gpx) oxidizes two GSH molecules to form the oxidized dimer GSSG for each reduction of $\mathrm{H}_{2} \mathrm{O}_{2}$. GSSG is then reduced to two GSH molecules through glutaredoxins ( $\mathrm{Grx}$ ) under the use of nicotinamide adenine dinucleotide phosphate (NADPH) as reduction equivalents (Figure 1 A). Similarly, $\operatorname{Trx}$ can act as reduction equivalents for peroxiredoxins ( $\operatorname{Prx})$ and other proteins. They reduce disulfide bonds by formation of an intramolecular disulfide, which is in turn reduced by thioredoxin-reductase (TrxR) in an NADPH dependent reaction (Figure $1 \mathrm{~B})$.

A

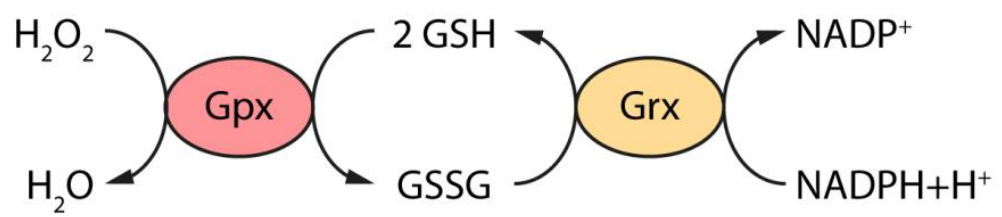

B

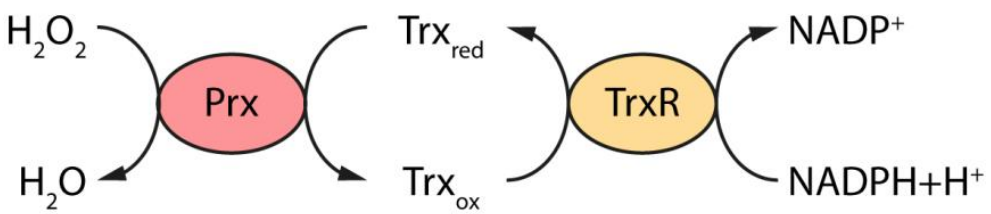

Figure 1 The GSH and Trx antioxidant systems.A) Reduced Glutathione (GSH) acts as a redox equivalent for Glutaperoxidases (Gpx). Oxidized glutathione (GSSG) is reduced by Glutaredoxin (Grx) with NADPH as electron donor. B) Thioredoxin (Trx) is oxidized when recovering Peroxiredoxins (Prx). The intramolecular disulfide is removed by Thioredoxinreductates $(\operatorname{Tr} \mathrm{R})$.

The cellular sources of ROS are manifold, but two dominant mechanisms have been described. One is the generation of superoxide in mitochondria due to electron transport chain leakage; the other is the generation of $\mathrm{O}_{2}{ }^{-}$and $\mathrm{H}_{2} \mathrm{O}_{2}$ by NADPH oxidases.

In actively respiring mitochondria, electrons are transferred via the complexes I to IV in the inner mitochondrial membrane in order to establish a proton gradient for adenosine triphosphate (ATP) production. Leakage of electrons leading to the reduction of molecular oxygen to superoxide can occur at all stages, but is most common at complex I and III (Murphy, 2009). The generated superoxide is then degraded via mitochondrial superoxide dismutases to $\mathrm{H}_{2} \mathrm{O}_{2}$. Although a side product, mitochondrial ROS are implicated to act in redox signaling and via complex crosstalk mechanisms. They provide a feedback loop between electron transport chain and substrate 
oxidation via the reaction with antioxidant NAD/NADH pools and can lead to so-called ROS induced ROS release into the cytosol (Zorov et al., 2000). This ROS induced ROS release can act as a pathologic amplifying mechanism in disease conditions, but has also been postulated to occur at baseline as a modulation of physiological ROS signaling and is then called superoxide flashes (Wang et al., 2008).

NADPH oxidases (NOX) are heterodimeric flavocytochromes with a gp22phox subunit. Seven NOX subtypes have been characterized in total of which NOX2 and NOX4 are the main isoforms found in cardiomyocytes. They produce $\mathrm{O}_{2}{ }^{--}$and/or $\mathrm{H}_{2} \mathrm{O}_{2}$ at defined localizations within the cell, leading to local protein modifications. NOX2 is activated by association with regulatory subunits such as $\mathrm{p} 47^{\text {phox }}, \mathrm{p} 67^{\text {phox }}, \mathrm{p} 40^{\text {phox }}$, and Rac1 and localized to the plasma membrane (Brandes et al., 2014). Activation occurs upon various stimuli via G-protein coupled receptor agonists, cytokines such as Ang 2 and mechanical stress and is related to apoptosis, interstitial fibrosis and hypertrophy (Bendall et al., 2002; Looi et al., 2008). In the physiological context, NOX2 derived $\mathrm{H}_{2} \mathrm{O}_{2}$ modulates $\mathrm{Ca}^{2+}$ release by ryanodine receptor 2 (RyR2) and $\mathrm{Ca}^{2+}$ re-uptake through sarco/endoplasmic reticulum $\mathrm{Ca}^{2+}$ ATPase (SERCA) as well as myofilament function and ion channel activity through modulation of kinase pathways such as CamKII, PKA, and protein kinase G1a (Santos et al., 2016).

NOX4 is thought to be constitutively active and its activity regulated by abundance and localization (Brandes et al., 2014). It is described to localize to perinuclear ER and mitochondria and plays a role in hypoxia, cardiac ischemia and pressure overload (Santos et al., 2016).

Other ROS sources in cardiomyocytes include uncoupled NO synthases, xanthine oxidases (XO) and mitochondrial monoamino oxidases (MAO). Uncoupling of NO synthases occurs when the cofactor tetrahydrobiopterin (BH4) is depleted or by S-glutathionylation (Chen et al., 2010; Xia et al., 1998). As BH4 depletion is amplified by ROS, NOS uncoupling could be a further mechanism of potentiation of ROS signaling. As uncoupling can occur also partially, it leads to the concomitant production of superoxide and nitric oxide, which react to form peroxinitrite, ONOO-, leading to nitroxidative stress and protein nitration. XO catalyzes the oxidation of hypoxanthine to xanthine and further to uric acid. In this process, electrons are transferred to oxygen and water, generating $\mathrm{O}_{2}{ }^{-}$and $\mathrm{H}_{2} \mathrm{O}_{2}$. Heart failure seems to be associated with an upregulation of XO (de Jong et al., 2000). Furthermore, interactions of XO derived ROS with NO signaling have been implicated in RyR2 $\mathrm{Ca}^{2+}$ leak observed in heart failure (Gonzalez et al., 2010). Although pharmacological inhibition of XO has been beneficial in animal models (Naumova et al., 2006), large scale clinical trials did not report benefits of XO inhibition in patients with heart failure (Givertz et al., 2015). Recently, mitochondrial MAOs have been shown to play important roles in cardiovascular pathology (Deshwal et al., 2017). MAOs are 
membrane bound enzymes in the outer mitochondrial membrane, which deaminate neurotransmitters and other amines. In these processes, $\mathrm{H}_{2} \mathrm{O}_{2}$ can be generated as a side product with an impact on mitochondria redox balance (Kaludercic et al., 2014).

All the mechanisms described above contribute to the cellular redox homeostasis, which describes the balances between ROS production and antioxidant mechanisms. This balance is crucial for correct cardiomyocyte function. Disturbances in the regulation of oxidative and antioxidative balances have been shown to lead to cardiac dysfunction and disease. A cardiac pathology, where the involvement of ROS is well studied, is myocardial infarction. In myocardial infarction, arterial occlusion leads to a drop in perfusion and lack of oxygen supply to the affected tissue. As a consequence, mitochondrial respiration ceases and electrons that cannot be used for ATP synthesis accumulate. This leads to an increased production of ROS, which in turn can lead to opening of the mitochondrial permeability transition pore (mPTP) (Kim et al., 2006). The mPTP is a non-specific channel in the inner mitochondria membrane, which molecules up to $1.5 \mathrm{kDa}$ can pass upon rapid opening. mPTP opening can lead to the release of $\mathrm{Ca}^{2+}$ and cytochrome $\mathrm{c}$ into the cytosol, which triggers cardiomyocyte dysfunction and apoptosis (Crompton, 1999). Furthermore, in ischemia energy production switches to glycolysis and ATP production rates decrease, leading to a decline in cellular energy levels. ATP reliant ion transporters such as $\mathrm{Na}^{+} / \mathrm{K}^{+}$ATPase or SERCA are inhibited, leading to disturbances in cellular ion homeostasis characterized by an accumulation of intracellular $\mathrm{Ca}^{2+}$ (Paradis et al., 2016). Increased $\mathrm{Ca}^{2+}$ further stimulates mPTP opening, which releases more $\mathrm{Ca}^{2+}$ into the cytosol and leads to the hydrolysis of ATP by uncoupled F-ATPase, leading to an amplification of cellular damage.

Reperfusion is crucial for cardiomyocyte survival, but initially leads to an amplification of damage. When circulation is re-established, the sudden availability of oxygen leads to a further increase in ROS, due to XO activity, impaired mitochondrial respiration and depleted antioxidant pools (Zhou et al., 2018). These ROS signals can further increase the mitochondrial damage via mPTP opening leading to mitochondrial swelling and rupture and thus activation of apoptotic pathways (Lesnefsky et al., 2017).

Interestingly, reperfusion associated injury can be reduced by transient hypoxic episodes prior to prolonged ischemia. This phenomenon is termed ischemic preconditioning and has been strongly related to ROS signals. It is thought that the various pathways involved in this process converge on inhibition of mPTP opening, potentially by activation of protein kinase $C$ through ROS derived from mitochondria and NOX2 (Yang et al., 2010). Furthermore, the beneficial effect of ischemic preconditioning can be observed for more than $24 \mathrm{hrs}$ with the late phase mediated by an 
upregulation of hypoxia inducible factor $1 \alpha$ (Hif1 $\alpha$ ) target genes such as inducible NO synthase or heme oxygenase-1 (Guo et al., 1999; Yoshida et al., 2001).

Considering the important role of ROS in cardiovascular injury, the idea of ROS-scavenging therapies might seem appealing. Indeed, high plasma levels of antioxidant vitamins such as vitamin C, vitamin E or polyphenols are often associated to reduce cardiovascular disease risk (Dauchet et al., 2009). However, studies of dietary vitamin supplementation have failed to show clinical benefits in the prevention of cardiovascular disease (Myung et al., 2013). In addition, therapeutic approaches "flooding" the organism with antioxidants do not make sense in light of physiologic roles of ROS signaling, which is required for proper cardiomyocyte function. Nonetheless there is data from small scale clinical trials using antioxidant administration before angioplasty in patients with myocardial infarction. The rationale behind these approaches is the prevention of reperfusion injury by prevention of ROS bursts upon re-oxygenation of the infarcted tissue. In this context ascorbate infusion was shown to improve microvascular perfusion and left ventricular ejection fraction, but had no effect on infarct size (Basili et al., 2010; Ramos et al., 2017; Valls et al., 2016). Administration of radical scavenger Edavarone reduced infarct size and the incidence of arrhythmias in patients undergoing angioplasty (Tsujita et al., 2006). Despite these small scale studies reporting beneficial effects, large scale clinical trial data is missing.

In light of the highly regulated nature of ROS signaling, recent studies have focused on the use of more targeted ROS modulation. For example, a preclinical guinea pig study found that the administration of mitoTEMPO, a mitochondria targeted ROS scavenger, can rescue heart failure induced by chronic catecholamine stimulation and pressure overload (Dey et al., 2018). It stands to reason that for these therapeutic approaches more knowledge about the spatial and temporal regulation of ROS signals is needed.

\subsection{Methods to detect and measure ROS}

One of the major challenges in redox research has been the detection of cellular ROS with high sensitivity and good spatial resolution. Traditional approaches have used dye based assays where reaction of ROS with a dye molecule leads to changes in its fluorescence, absorbance or light production. Widely used assays include dichlorodihydrofluorescein diacteate (H2DCF-DA), Amplex Red or dihydroethidium (DHE) probes.

H2DCF-DA is a membrane permeable molecule, which is cleaved by cellular esterase upon entry and thus retained in cells. That way H2DCF can accumulate intracellularly with a tendency of enrichment in mitochondria (Rezende et al., 2018). Upon reaction with single electron oxidants such as $\mathrm{O}_{2}^{-}$or 
$\mathrm{H}_{2} \mathrm{O}_{2}$, fluorescent DCF is generated and can be detected by excitation at $495 \mathrm{~nm}$ and emission at $529 \mathrm{~nm}$. As the reaction of H2DCF to DCF is not specific to a single oxidant, the assay cannot give a clear answer as to the source of ROS observed. In addition, H2DCF is sensitive to oxidation by heme, $\mathrm{NO}_{2}$ and light (Marchesi et al., 1999).

In contrast to H2DCF, horse raddish peroxidase (HRP) coupled assays such as Amplex Red provide specificity towards $\mathrm{H}_{2} \mathrm{O}_{2}$ mediated oxidation. In the presence of $\mathrm{HRP}$ and $\mathrm{H}_{2} \mathrm{O}_{2}$, Amplex Red is oxidized to resorufin, a molecule fluorescent at $590 \mathrm{~nm}$ emission upon excitation at $530 \mathrm{~nm}$ (Zhou et al., 1997). As Amplex Red does not enter cells, the assay is strictly extracellular, but can be used with isolated organelles, e.g. mitochondria. It is a highly sensitive assay but can react with light, peroxynitrite-derived radicals and mitochondrial carboxylesterase (Dębski et al., 2016; Miwa et al., 2016; Zhao et al., 2012).

DHE can be used to detect superoxide. It has been shown that DHE reacts via a radical intermediate to 2-Hydroxyethidium $\left(2-\mathrm{OH}-\mathrm{E}^{+}\right)$, a highly fluorescent substrate. However, analysis of 2-OH-E fluorescence can be confounded by other fluorescent side products such as ethidium that are generated by unspecific oxidations of DHE. Therefore, the most reliable detection of $2-\mathrm{OH}-\mathrm{E}^{+}$is based on high performance liquid chromatography (Zielonka \& Kalyanaraman, 2010). What makes DHE attractive for superoxide measurements is the possibility to target the probe to mitochondria. mitoSOX ${ }^{\mathrm{TM}}$ allows for the specific detection of mitochondrial $\mathrm{O}_{2}^{-}$by generation of mito-2-OH-E+ (Robinson et al., 2006). As most dye based assays, also DHE is prone to oxidation by light, heme oxidases and auto-oxidation.

A common feature of dye based assays is the accumulation of signal over time by irreversible oxidation of probe molecules. Although this mechanism leads to high sensitivity of ROS detection, it masks reversible ROS production and dynamic patterns of ROS bursts. Furthermore, phototoxicity and bleaching make long term measurements difficult.

These issues can be overcome by recently developed fluorescent protein redox sensors. In general, these sensors are based on fluorescent proteins and will be produced by the cell of interest. They can easily be targeted to individual organelles by inclusion of signal peptides in the sensor coding sequence. They react with cellular ROS species in a reversible manner and can thus report dynamic changes in cellular ROS. In this study, we made use of two different fluorescent sensor systems, the GSH sensor Grx1-roGFP2 and the $\mathrm{H}_{2} \mathrm{O}_{2}$ sensor HyPer.

Grx1-roGFP2 was developed based on enhanced green fluorescent protein (eGFP). Wild type GFP can exist at two different protonation states, the protonated A-band and the deprotonated B-band. 
The A-band can be excited at $405 \mathrm{~nm}$, while the B-band is excited at $488 \mathrm{~nm}$. Excited state proton transfer leads to a deprotonation of the excited A-band by transfer of $\mathrm{H}^{+}$to a neighboring amino acid outside of the fluorophore ring. This process leads to emission at the same wavelength of $510 \mathrm{~nm}$ for both protonation states (Figure 2 A) (Brejc et al., 1997; Chattoraj et al., 1996). In eGFP a S65T mutation leads to the stabilization of the protonated B-band and thus optimized fluorescence properties (Heim et al., 1995). When cysteines are introduced to the fluorescent barrel structure, disulfide bonds can form upon oxidation, leading to a conformational change that influences the protonation state and thus the fluorescent properties of the molecule. In case of roGFP2, S147C and Q204C mutations lead to a shift of excitation wavelength from $488 \mathrm{~nm}$ (protonated B-band) to $405 \mathrm{~nm}$ (deprotonated A-band) upon oxidation (Figure $2 \mathrm{~B}$ ) (Hanson et al., 2004). If expressed in the cell as roGFP2 alone, the equilibration to cellular oxidants is too slow and unspecific. By fusion of roGFP2 to redox relay proteins, specificity and fast equilibration can be achieved. Fusion to Grx1 for example gave rise to redox sensor Grx1-roGFP2, which is highly specific for cellular GSH (Gutscher et al., 2008). The reaction of Grx1-roGFP2 with cellular GSH/GSSG is fully reversible, which allows for the detection of dynamic changes of GSH/GSSG (Figure $2 \mathrm{C}$ ). Due to the ratiometric properties of the sensor, transgene expression level and photo bleaching do not compromise measurements. Other variants of roGFP2 based sensors include roGFP2-Orp1, which gains specificity to $\mathrm{H}_{2} \mathrm{O}_{2}$ induced oxidation from Orp1, a yeast peroxidase (Gutscher et al., 2009) and peroxiredoxin based probes roGFP2-Tsa2 $\Delta C R$ and roGFP2-Tsa2 $\triangle C P \Delta C R$ (Morgan et al., 2016). 
A

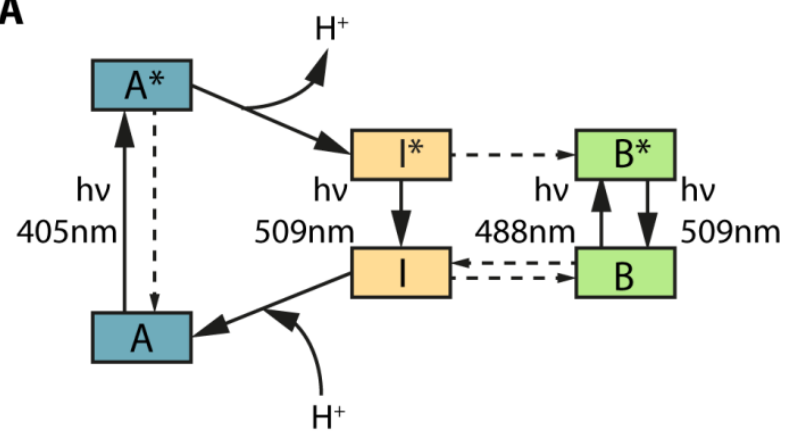

B

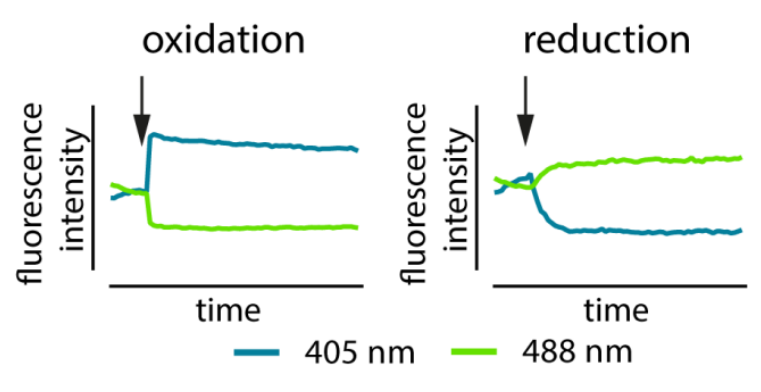

C

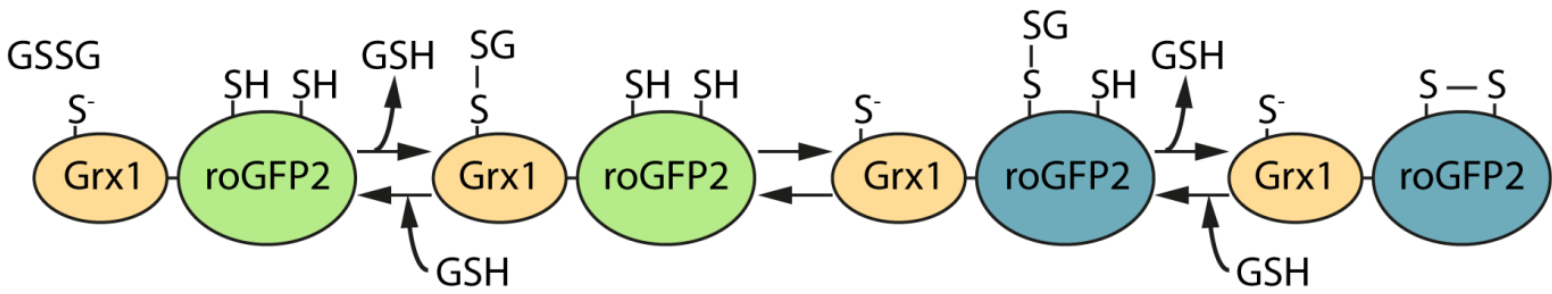

D
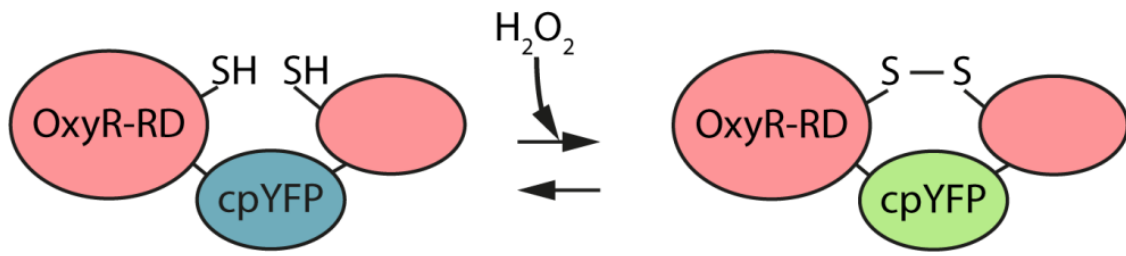

Figure 2 Basic principle of fluorescent protein redox sensors. A) Fluorochrome protonation influences the excitation wavelength preference of fluorescent proteins, demonstrated here for GFP. The protonated A band is excited by $405 \mathrm{~nm}$ light, whereas the deprotonated B band is excited by $488 \mathrm{~nm}$ light. Both forms emit light at $509 \mathrm{~nm}$, as excited state proton transfer to neighboring amino acids leads to the deprotonation of the excited A-band fluorophore. B) Redox sensitive mutations of fluorescent proteins change excitation wavelength upon oxidation or reduction. Fluorescence intensity over time after excitation with either $405 \mathrm{~nm}$ (blue) or $488 \mathrm{~nm}$ (green) light of roGFP2. Arrows indicate the point of addition of oxidative or reducing agents. C) Principle of Grx1-roGFP2 GSH dependent oxidation and reduction. Grx1-roGFP2 reacts with the cellular GSH/GSSG pool in a dynamic, reversible manner. Disulfide formation upon oxidation leads to a shift of excitation wavelength maxima. D) Principle of $\mathrm{H}_{2} \mathrm{O}_{2}$ sensor HyPer. cpYFP is integrated into bacterial $\mathrm{H}_{2} \mathrm{O}_{2}$ response element OxyR-RD. Upon reaction of OxyR-RD with $\mathrm{H}_{2} \mathrm{O}_{2}$, disulfide formation leads to major conformational changes resulting in an excitation wavelength shift of cPYFP.

Another well studied $\mathrm{H}_{2} \mathrm{O}_{2}$ sensor is HyPer. HyPer was generated by insertion of a circularly permutated yellow fluorescent protein (YFP) between amino acids 205-206 of bacterial OxyR regulatory domain (OxyR-RD) (Belousov et al., 2006). OxyR is a bacterial transcription factor sensitive to $\mathrm{H}_{2} \mathrm{O}_{2}$ mediated oxidation. Upon reaction of the regulatory domain with an oxidant, major conformational changes lead to the initiation of redox defense gene transcription (Choi et al., 2001; Zheng et al., 1998). In case of Hyper, an oxidation of OxyR-RD leads to a shift in fluorescence properties of cpYFP (Figure 2 D). Similarly to GFP, YFP possesses two excitation maxima at $420 \mathrm{~nm}$ and $500 \mathrm{~nm}$ and one emission maximum at $516 \mathrm{~nm}$. Oxidation of OxyR-RD leads to a decrease of fluorescence upon $420 \mathrm{~nm}$ excitation and a concomitant increase of fluorescence upon $500 \mathrm{~nm}$ excitation. The reduction of HyPer is most likely achieved via cellular GSH as demonstrated for wild 
type OxyR in E.coli (Zheng et al., 1998). To ensure the specificity of the observed change in fluorescence, the redox active cysteines in OxyR-RD, Cys199 and Cys208 were mutated to serines. The resulting redox dead variants of $\mathrm{HyPer}$ do not react to $\mathrm{H}_{2} \mathrm{O}_{2}$, but can be influenced by changes in pH (Belousov et al., 2006). The C199S mutant is now known as cellular pH sensor SyPher and can be used as a suitable control in HyPer experiments (Matlashov et al., 2015). Newer versions of HyPer have been developed by further optimization of fluorescent properties. These variants termed HyPer2 and HyPer3 possess a larger dynamic range, stronger fluorescence and less $\mathrm{pH}$ sensitivity (Bilan et al., 2013; Markvicheva et al., 2011). In addition to the cpYFP based green sensors, a HyPerRed variant based on cpRed, a circularly permutated mApple, was developed. In contrast to the classical green HyPer probes, HyperRed is not ratiometric but rather intensiometric with an increase of fluorescence intensity upon oxidation by $\mathrm{H}_{2} \mathrm{O}_{2}$ (Ermakova et al., 2014). Another interesting application of HyPer has been the fusion to a D-amino acid oxidase. This HyPerDAO fusion protein can act as a sensor producer hybrid, with DAO producing $\mathrm{H}_{2} \mathrm{O}_{2}$ when supplied with Damino acids (Matlashov et al., 2014). Since intracellular production of $\mathrm{H}_{2} \mathrm{O}_{2}$ is very likely to have distinct effects from a general application of oxidants to the cell culture medium, this is an intriguing possibility to manipulate cellular redox homeostasis.

Fluorescent protein based redox sensors offer many advantages over dye based systems, but they are inferior in the sensitivity towards oxidants. Most fluorescent protein based sensors can detect oxidants in the high nanomolar range. This includes physiological signals such as oxidative bursts but might not be sensitive enough for the detection of small scale ROS events such as very confined local ROS "sparks" in cellular microdomains, which might just be in the picomolar range.

Examples of application of fluorescent protein based sensors have demonstrated that these sensors can indeed detect physiological ROS signals. Cell based assays have shown ROS involvement in apoptosis and growth factor signaling (Belousov et al., 2006). Recently, mice expressing Grx1-roGFP2 or its mitochondria targeted version under the control of the cardiac $\alpha$-myosin heavy chain ( $\alpha \mathrm{MHC})$ promotor were used to investigate cellular redox potential in isolated adult myocytes (Swain et al., 2016). Interestingly, in this study cardiac mitochondria possessed a more reduced GSH/GSSG pool compared to cytosol. Furthermore, cytoplasmic oxidation after stimulation with isoprenalin and Ang 2 could be visualized, as well as hypoxia-reoxygenation induced changes in cellular redox homeostasis. In a similar study, Orp1-roGFP2 and Grx1-roGFP2 were expressed in the developing zebrafish vasculature and heart. Live fluorescence imaging of transgenic fish embryos $48 \mathrm{hpf}$ revealed differences in redox homeostasis between compartments, with mitochondria being more oxidized than cytosol or nucleus in all analyzed cell types. By inhibiting either pentose-phosphate- 
pathway or GSH synthesis, the involvement of metabolic pathways in cellular redox homeostasis was demonstrated (Panieri et al., 2017). While these two studies focused on the applicability of genetically encoded redox sensors in animal models, other studies have used redox sensors to complement their findings. In a guinea pig model of non-ischemic heart failure, adeno-associated virus serotype 9 (AAV9) mediated expression of Orp1-roGFP2 and mito-Orp1-roGFP2 was used to investigate changes in redox homeostasis in failing and non-failing hearts. Non-ischemic heart failure led to an increase in both cytosolic and mitochondrial ROS that could be rescued by application of the mitochondria targeted ROS scavenger mitoTEMPO (Dey et al., 2018). HyPer and two variants targeted to either caveolae or mitochondria were used in NOX4 and NOX2 knockout mice to investigate insulin induced ROS signals. $\mathrm{H}_{2} \mathrm{O}_{2}$ appeared to be produced in caveolae upon insulin stimulation in cardiomyocytes from wild type and NOX4 knockout mice, while wild type and NOX2 knockouts demonstrated a cytosolic increase of $\mathrm{H}_{2} \mathrm{O}_{2}$ (Steinhorn et al., 2017). These results demonstrate how locally and temporally defined redox signals can be investigated using fluorescent protein redox sensors. However, they are restricted to animal models or stable human cell lines, which possess limitations in translation of findings to human pathology. Investigation of human cardiac redox biology needs suitable model systems that can also recapitulate complex cell-cell and cell-matrix interactions, which is not achieved in traditional cell culture assays. One such model system could be engineered heart muscles generated from stem cell derived cardiomyocytes and stromal cells.

\subsection{Stem cells and engineered tissues as model systems}

Pluripotent stem cells are cells that possess an unlimited self-renewal capacity and are able to differentiate into derivatives of all three germ layers. Embryonic stem cells as the classic pluripotent stem cell source are generated by isolation and expansion of inner cell mass of the preimplantation embryo under suitable growth conditions (Thomson et al., 1998). To avoid ethical concerns inherent to embryonic stem cells, protocols for the induction of pluripotency in adult differentiated cells were generated. Overexpression of four different transcription factors, Oct4, Klf2, Sox2, and c-myc is sufficient to reprogram cells back to a pluripotent fate (Takahashi et al., 2007; Takahashi \& Yamanaka, 2006). Delivery of these four "Yamanaka factors" can be achieved for example by viral transduction, plasmid DNA transfection, protein electroporation or modified messenger RNA. As induced pluripotent stem cells (iPSCs) can be generated from apparently every individual, they are a very promising tool for the development of personalized therapies, screening platforms and regenerative medicine. 
Furthermore, the rise of gene editing technologies like transcription activator-like effector nucleases (TALEN) or clustered regularly interspaced short palindromic repeats (CRISPR)/Cas9 has even added to the possibilities of stem cell use in model systems and therapies. Both technologies introduce mutations or target gene insertions by initially inducing a DNA double strand break at a defined location. If double strand breaks are repaired by non-homologous end joining, short insertions or deletions (so called indels) can lead to frameshifts and subsequent loss of protein function. When a donor fragment with homology sequences is supplied, homology directed repair will lead to the integration of donor sequences. Through this transgenes can be inserted or genomic sequences replaced. This allows also for the replacement of "defective" genes with corrected sequences, which can be used to verify disease causing mutations in patient derived IPSC of varying genetic background. Historically, the first technology developed for targeted double strand breaks were engineered zinc-finger nucleases. They consist of an array of six zinc-finger DNA binding domains, each recognizing a three basepair triplet fused to a Fokl endonuclease. In order to cut DNA, FokI needs to dimerize. This requires the design of two Zinc-finger nucleases, binding the targeted DNA sequence in close proximity (Urnov et al., 2010). Similar to zinc-finger technology are TALENs. TALEs are proteins from the bacteria species Xhanthomonas with a DNA binding domain consisting of several 33-35 amino acid repeat domains. DNA binding is mediated via the repeat-variable di-residue (RVD) within this repeat domain. Each RVD binds a specific DNA base, thereby; TALEs can be engineered to recognize any basepair sequence on DNA (Boch et al., 2009). Fusion of a Fokl endonuclease domain then confers DNA cutting (Christian et al., 2010; Miller et al., 2011). Also TALENs need to dimerize on the target sequence to initiate DNA cleavage. Although TALENs work very similar to zinc finger nuclease, they were described to have less cytotoxic side effects when used for genome editing (Mussolino et al., 2011).

In contrast to dimerizing nucleases, CRISPR/Cas9 protein can cut DNA directly when binding to the target sequence. CRISPRs are RNA-protein complexes of CRISPR protein, a tracrRNA, and a crRNA, the latter giving binding specificity (Cong et al., 2013; Jinek et al., 2012; Mali et al., 2013). As only a complementary RNA sequence is required for targeting of CRISPRs, the design is simplified in comparison to zinc-finger nucleases or TALENs. In addition, CRISPR/Cas9 is cutting DNA with a higher efficiency than TALENs, which can also be a downside with more unspecific sequences also targeted. Recent reports have highlighted the activation of p53 DNA damage response upon Cas9 induced DNA double strand breaks, a process that might increase CRISPR/Cas9 toxicity and inhibit efficient transgene integration (Haapaniemi et al., 2018; Ihry et al., 2018). Advances in the delivery of CRISPR/Cas9 as ready formed ribonucleoprotein complexes have reduced off-target effects and cytotoxicity (Farboud et al., 2018). Notably, the high efficiency of CRISPR DNA binding can also be 
used for other applications. So-called nuclease-dead variants without DNA cutting properties have been fused to transcriptional activators or epigenetic modulators to manipulate and fine-tune gene expression patterns (La Russa \& Qi, 2015; Xu \& Qi, 2019). While these applications are still being developed and optimized, they offer exciting possibilities for future research and therapy.

Soon after the establishment of stem cell culture, protocols for the differentiation into various cell types were developed. Early differentiation protocols assemble cells in hanging drops to form 3D embryoid bodies mimicking the physiological environment of these cells. In recent years, many of these protocols have been adapted to 2D culture conditions. By supplementation with different growth factors and small molecules, signaling pathways are fine-tuned to enrich cell types of interest. In cardiac differentiation it is known from embryology that cardiac mesoderm specification is driven by activation of wht and bone morphogenetic protein (BMP) signaling, while subsequent cardiac specification relies on the inhibition of wnt signaling (Später et al., 2014). Typically, mesoderm specification is induced in culture by treatment with recombinant BMPs (Burridge et al., 2011) and CHIR, a small molecule inhibitor of glycogen synthase kinase $3 \beta$, which, in turn, is a wnt inhibitor (Bennett et al., 2002). Subsequent wnt inhibition is achieved by supplementation with small molecules of the inhibitor of wnt production (IWP) family, typically IWP2 or IWP4. IWPs inhibit porcupine, an O-acyltransferase necessary for palmitoylation, and thus stability and secretion of wnt (Chen et al., 2009). Further enrichment of cardiomyocytes can then be achieved by metabolic selection with glucose free, lactate rich media, as lactate is preferably metabolized by cardiomyocytes. These classical differentiation protocols give rise to predominantly ventricular cardiomyocytes (Burridge et al., 2014; Lian et al., 2013; Weng et al., 2014). Adaptations have been developed to enrich other cardiomyocyte subtypes, such as atrial myocytes, which can be induced by additional treatment with retinoic acid (Cyganek et al., 2018; Devalla et al., 2015; Laksman et al., 2017; Lee et al., 2017).

One major issue pertaining to the differentiation of cardiomyocytes is the lack of full maturation of these cells. Stem cell derived cardiomyocytes usually attain a fetal phenotype. They express lower amounts of key $\mathrm{Ca}^{2+}$ handling proteins such as RyR2, SERCA, and calsequestrin 2 and show expression of fetal sarcomeric isoforms myosin heavy chain 6 (MYH6) and titin N2A (van den Berg et al., 2015). In contrast to isolated adult cardiomyocytes morphology is more rounded with shorter, less aligned sarcomeres and rarely t-tubules (Gherghiceanu et al., 2011; Lundy et al., 2013). In addition, metabolism and electrophysiological properties differ further from an adult phenotype. Different strategies have been developed to increase the maturation of stem cell derived cardiomyocytes. Supplementation of hormones like thyroid hormone triiodothyronine or 
glucocorticoid analog dexamethasone to culture media has been tested in several studies (Parikh et al., 2017; Yang et al., 2014); however the physiological relevance of these signaling molecules in cardiac maturation is not clear. Considering metabolism, stem cells and stem cell derived cardiomyocytes rely on a mainly glycolysis driven ATP generation whereas adult cardiomyocytes are characterized by a preference for fatty acid oxidation and oxdidative phosphorylation (Lopaschuk \& Jaswal, 2010; Makinde et al., 1998). Supplementation of culture media with fatty acids and stimulation of oxidative phosphorylation by small molecule inhibition of Hif1 $\alpha$ or lactate dehydrogenase A has shown to improve stem cell derived cardiomyocyte metabolism (Correia et al., 2017; Hu et al., 2018). Also electrical stimulation of monolayer cardiomyocytes can be used in order to improve cellular $\mathrm{Ca}^{2+}$ handling (Kroll et al., 2017).

It has been suggested that cultivation in a $3 \mathrm{D}$ environment enhances cardiomyocyte maturation and function in-vitro (Tiburcy et al., 2011). Following this approach, cardiac tissue engineering focuses on the generation of multicellular cardiac organoids, by embedding cardiomyocytes in extracellular matrix substrates. One very commonly used matrix in this process is collagen type I (Soong et al., 2012; Tiburcy et al., 2014). For the development of functional heart muscle, a stromal cell type is necessary (Naito et al., 2006). It has been postulated that stromal cells help supply a cardioinstructive environment (Christalla et al., 2012) and it was demonstrated that matrix remodeling by stromal cells is an integral part of early tissue formation (Schlick et al., 2018). Others have also supplemented cardiac organoids with endothelial cells, in order to achive vascular network formation (Tulloch et al., 2011). Apart from the cellular composition, the biomechanical cues delivered to the tissue also seem to impact force development and cardiomyocyte maturation. Cyclic stretch as well as passive stretch induced by different casting geometries seem to improve force generation and cardiomyocyte maturation (Abilez et al., 2018; Tulloch et al., 2011; Zimmermann et al., 2002, 2006). Further improvement of culture conditions include application of electrical stimulation regimens (Godier-Furnémont et al., 2015; Radisic et al., 2004; Ronaldson-Bouchard et al., 2018) or modulation of metabolic supply of glucose and palmitate (Mills et al., 2017). Engineered heart muscle allows for the direct determination of generated force and tissue stiffness, important characteristics of native myocardium. Applications also include disease modelling, drug screening and regeneration (Eder et al., 2014; Jacob et al., 2016; Meyer et al., 2019; Tiburcy et al., 2017; Voges et al., 2017). Disease modelling with patient derived iPSC has been demonstrated for a patient with dilative cardiomyopathy caused by mutations in RNA-binding motif protein20, where the patient engineered heart muscle (EHM) phenotype was characterized by less force of contraction and less passive stress (Streckfuss-Bömeke et al., 2017). In another study, a gene therapy approach for Duchenne's muscular dystrophy (DMD) based on exon skipping has been shown to rescue the DMD 
phenotype in EHM (Long et al., 2018). These studies and others demonstrate how engineered heart muscle models can be used as a versatile tool to investigate cardiac physiology and disease. This is even enhanced by the possibility to generate tissues in multi-well platforms with optical read-out of heart muscle function in order to streamline and enhance screening applications (Jacob et al., 2016; Mills et al., 2017; Nunes et al., 2013).

Apart from screening and disease modeling applications, engineered tissues also hold promising perspectives for the therapy of heart disease. EHM have been shown to improve cardiac function after myocardial infarction in rat and guinea pig models (Riegler et al., 2015; Weinberger et al., 2016; Zimmermann et al., 2006). Since tissue constructs could be made in any desired geometry, also applications as biological ventricular assist devices of pouch shaped constructs are possible (Yildirim et al., 2007). Although technical hurdles to the clinical realization of these ideas still exist, they might provide a solution to the increasing demand for transplantable organs in the future. 


\section{Aims of the thesis}

Redox signaling is an integral part of cardiac physiology. With stem cell derived cardiomyocytes and engineered tissues, we have very versatile tools to investigate redox signaling in single cells and cellcell interactions. Application of fluorescent redox sensors in human stem cell derived cardiomyocytes had previously been demonstrated in our lab (Heta, 2017). In order to fully take advantage of redox sensor technology on all levels of our EHM generation process from pluripotent stem cell to cardiomyocyte to EHM, we sought to:

(1) Generate human pluripotent stem cell lines with stable expression of $\mathrm{GSH}$ and $\mathrm{H}_{2} \mathrm{O}_{2}$ sensors;

(2) Compare redox homeostasis between stem cells and stem cell derived cardiomyocytes in respect to cytosolic and mitochondrial GSH/GSSG pools;

(3) Establish models of $\mathrm{H}_{2} \mathrm{O}_{2}$ sensing and production in human pluripotent stem cells and stem cell derived cardiomyocytes. 


\section{Material \& Methods}

\subsection{Cell culture}

\subsubsection{TSA201}

TSA 201 cells (ECCAC) were grown in Dulbeccos modified Eagle's medium high glucose $(4.5 \mathrm{~g} / \mathrm{l}$, DMEM) supplemented with $5 \%$ fetal calf serum (FCS) on standard cell culture vessels. Splitting was performed with $0.05 \%$ Trypsin-EDTA when cells reached confluency. Coverslips and imaging plates were coated with $0.1 \%$ gelatin in sterile $\mathrm{H}_{2} \mathrm{O}$ for $20 \mathrm{~min}$ at room temperature prior to cell seeding. Cells were seeded at $0.05 * 10^{6}$ cells $/ \mathrm{cm}^{2}$.

\subsubsection{HFF}

Human foreskin fibroblasts (HFF) were grown in high glucose DMEM supplemented with 15\% FCS and $100 \mathrm{U} / \mathrm{mL}$ penicillin, $100 \mu \mathrm{g} / \mathrm{mL}$ streptomycin. Cells were split using TrypLE digestion. In general, passages 20 to 30 were used for experiments.

\subsubsection{HES2}

Human embryonic stem cells (HES2, ESI Singapore) were grown on irradiated HFF as feeder layers in Knock-out DMEM supplemented with 20\% Knock-out Serum Replacement, $2 \mathrm{mM}$ glutamine, 1\% non-essential amino acids (NEAA), $100 \mathrm{U} / \mathrm{mL}$ penicillin, $100 \mu \mathrm{g} / \mathrm{mL}$ streptomycin, and $10 \mathrm{ng} / \mu \mathrm{l}$ fibroblast growth factor 2 (FGF2). Cells were split using TrypLE, resuspendend in HES medium supplemented with $5 \mu \mathrm{M}$ rho-associated kinase (ROCK) inhibitor Y27632 (Stemgent) and seeded at $4 * 10^{3}$ cells $/ \mathrm{cm}^{2}$ for subculture. ROCK inhibitor was included until $24 \mathrm{~h}$ after seeding. E8 medium (stem cell technologies) was used for feeder free cultures and differentiation on 1:120 Matrigel ${ }^{\mathrm{TM}}$ in sterile phosphate buffered saline (PBS) coated cell culture vessels.

\subsection{4. hCM}

Differentiation of pluripotent stem cells to human cardiomyocytes (hCM) was performed as previously described (Tiburcy et al., 2017). Briefly, stem cells where seeded under feeder free conditions at optimized densities on differentiation day -4 in E8. Mesoderm differentiation was induced at d0 by a combination of activin A, BMP4, CHIR and FGF2 in serum free Roswell Park Memorial Institute 1640 (RPMI 1640) medium (RPMI 1640 supplemented with 2\% B27, 200 mM Lascorbic acid and $100 \mathrm{U} / \mathrm{mL}$ penicillin, $100 \mu \mathrm{g} / \mathrm{mL}$ streptomycin). From d4 to d13, serum free RPMI medium was supplemented with $5 \mu \mathrm{M}$ IWP4. Cardiomyocytes were purified by metabolic selection in glucose free lactate containing medium. Differentiation ended at $d 21$. For subsequent experiments, hCM were dissociated by incubation with Accutase, $0.025 \%$ trypsin, $20 \mu \mathrm{g} / \mathrm{ml}$ DNasel 
for $20 \mathrm{~min}$ at room temperature and reseeded onto 1:120 Matrige $^{\mathrm{TM}}$ in PBS coated cell culture vessels.

\subsubsection{EHM}

Engineered heart muscle was generated as previously described (Tiburcy et al., 2017). EHM used in this study were kindly generated by S. Schlick and S. Nagel, both Institute of Pharmacology and Toxicology.

\subsection{Cloning}

For all cloning approaches, insert coding sequences were amplified by polymerase chain reaction (PCR) using Primestar-HS (takara) or ExTaq (takara) DNA polymerase according to the manufacturer's protocols. Through the use of carefully designed primers, restriction endonuclease recognition sites were integrated at the $5^{\prime}$ and $3^{\prime}$ end. For ExTaq PCR, up to 500 ng or template DNA were mixed with $5 \mu \mathrm{l} 10 \mathrm{x}$ buffer, $1 \mu \mathrm{l}$ of each forward and reverse primer $(10 \mu \mathrm{M}), 4 \mu \mathrm{l} 2.5 \mathrm{mM}$ dNTPs and $0.25 \mu \mathrm{l}$ ExTaq $(5 \mathrm{U} / \mu \mathrm{l})$. Volume was adjusted to $50 \mu \mathrm{l}$ using $\mathrm{H}_{2} \mathrm{O}$. PCR was run in a standard table top thermocycler according to Table 1. Primestar-HS PCR was carried out using up to 200 ng template DNA with $10 \mu \mathrm{l} 5 x$ PrimeStar buffer $\mathrm{Mg}^{2+}, 4 \mu \mathrm{l} 2.5 \mathrm{mM}$ dNTPs, $1 \mu \mathrm{l}$ of each forward and reverse primer $(10 \mu \mathrm{M})$ and $0.5 \mu \mathrm{l}$ PrimeStar Polymerase in a total volume of $50 \mu \mathrm{l}$. A three step cycling protocol as outlined in Table 2 was used. A comprehensive list of primers used can be found in the Appendix (Table 7).

Table 1 Thermocycling protocol for ExTaq PCR

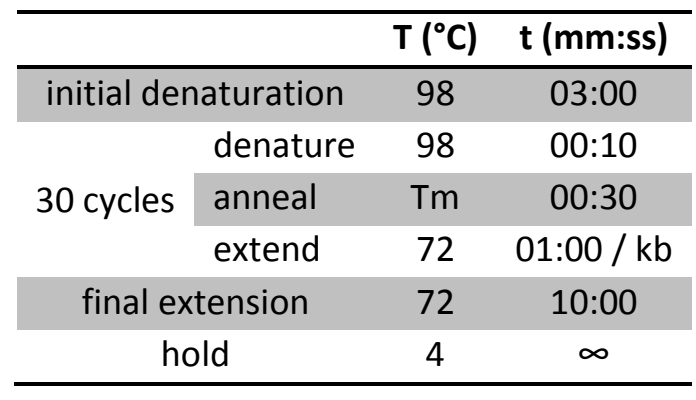

Table 2 Thermocycling protocol for PrimeStar-HS PCR

\begin{tabular}{cccc}
\hline & & $\mathbf{T ~}\left({ }^{\circ} \mathbf{C}\right)$ & $\mathbf{t}$ (mm:ss) \\
\hline \multirow{4}{*}{30 cycles } & denature & 98 & $00: 10$ \\
& anneal & $\mathrm{Tm}$ & $00: 05$ \\
& extend & 72 & $01: 00 / \mathrm{kb}$ \\
final extension & 72 & $05: 00$ \\
hold & 4 & $\infty$ \\
\hline
\end{tabular}


PCR products were run on 1 - 1.5\% agarose gels containing 1:200 Midori Green DNA dye and analyzed using a GelDoc XR imager with UV illumination and ImageLab software. Fragments were cut from the gel and cleaned using NucleoSpin Gel and PCR clean up kit. Restriction digest of up to $1 \mu \mathrm{g}$ of insert and vector DNA was carried out overnight at the incubation temperature recommended by the manufacturer, usually $37^{\circ} \mathrm{C}$ (see Table 3 for a list of enzymes used). Restriction enzymes were heat inactivated and DNA fragments either run on agarose gel and cleaned from cut out bands (backbone vectors) or directly cleaned from the digestion reaction (insert fragments). Ligation was carried out at $16^{\circ} \mathrm{C}$ overnight with T4 ligase (NEB). Reactions were set up according to manufacturer's instructions with molar ratios of backbone vector to insert of 1:3 up to 1:20. Ligation products were transformed into chemically competent bacteria (E. coli TOP10, prepared in-house) by heat shock. $50 \mu \mathrm{l}$ of competent bacteria were mixed with $5 \mu \mathrm{l}$ of ligation reaction and incubated for $30 \mathrm{~min}$ on ice. Heat shock was performed for $45 \mathrm{~s}$ at $42^{\circ} \mathrm{C}$, followed by addition of $500 \mu \mathrm{l}$ super optimal broth with catabolite suppression (SOC) medium and incubation at $37^{\circ} \mathrm{C}$ shaking at $300 \mathrm{rpm}$ for one hour. Bacteria were plated on lysogeny broth (LB) agar plates containing selection antibiotic (100 $\mu \mathrm{g} / \mathrm{ml}$ ampicillin) and incubated at $37^{\circ} \mathrm{C}$ overnight. Next day, colony PCR was carried out using FastGene Taq 2x Ready Mix. A PCR mastermix containing $5 \mu$ FastGene 2x Ready Mix, $0.2 \mu \mathrm{l}$ of each primer $(10 \mu \mathrm{M})$ and $4.6 \mu \mathrm{l} \mathrm{H}_{2} \mathrm{O}$ per reaction was prepared and $10 \mu \mathrm{l}$ added to each reaction tube. Colonies to screen were picked with a sterile pipette tip from which a copy plate was inoculated before the remaining bacteria were resuspended in PCR mastermix. Cycling was performed according to Table 4. PCR products were analyzed on a $1 \%$ agarose gel. When positive clones were detected, $5 \mathrm{ml}$ liquid LB culture containing selection antibiotic were inoculated with bacteria from the copy plate and grown overnight at $37^{\circ} \mathrm{C}$ on a shaking platform. DNA was purified from $5 \mathrm{ml}$ cultures with NuceloSpin mini Kit (macherey nagel) according to the manufacturer's protocol. Plasmid DNA was subjected to test digestion and sequencing for confirmation of correct insert integration. Successfully cloned constructs were further amplified by inoculation of $200 \mathrm{ml}$ liquid LB culture. After overnight incubation at $37^{\circ} \mathrm{C}$ on a shaking platform, DNA was purified with NucleoSpin midi kit and resuspended in up to $200 \mu \mathrm{l} \mathrm{H}_{2} \mathrm{O}$.

Table 3 List of restriction enzymes used. FD: Thermo Fisher fast digest formulation, HF: NEB high fidelity formulation

\begin{tabular}{lcccll}
\hline enzyme & sequence & $\begin{array}{c}\mathrm{T}\left({ }^{\circ} \mathrm{C}\right) \\
\text { incubation }\end{array}$ & $\begin{array}{c}\mathrm{T}\left({ }^{\circ} \mathrm{C}\right) \\
\text { inactivation }\end{array}$ & manufacturer & purpose \\
\hline Agel & ACCGGT & 37 & 65 & NEB & cloning \\
EcoRV-HF & GATATC & 37 & 65 & NEB & cloning \\
KspAI-FD & GTTAAC & 37 & 65 & thermo fisher & cloning \\
Notl-FD & GCGGCCGC & 37 & 80 & thermo fisher & cloning \\
Pacl & TTAATTAA & 37 & 65 & NEB & cloning \\
\hline
\end{tabular}




\begin{tabular}{lcllll}
\hline Sall-HF & GTCGAC & 37 & 65 & NEB & cloning \\
Sbfl & CCTGCAGG & 37 & 80 & NEB & test digest \\
Scal-FD & AGTACT & 37 & 65 & thermo fisher & linearization of donor vectors \\
Spel & ACTAGT & 37 & 80 & NEB & cloning \\
Xbal-FD & TCTAGA & 37 & 65 & thermo fisher & cloning \\
Xhol & CTCGAG & 37 & 65 & NEB & test digest \\
\hline
\end{tabular}

\subsection{1. pAAVS1 donor constructs}

A pAAVS1 homology site containing integration vector was obtained from system biosciences (cat\# GE602A-1). Previous to this study, the original phosphorglycerate kinase (PGK) promotor had been replaced by a CMV early enhancer chicken beta actin rabbit beta globin (CAG) promotor (pAAVS1-CAG). Furthermore, the GFP coding sequence had been removed by restriction digest with Fsel and $\mathrm{BmgBI}$ and subsequent vector relegation. The remaining base pairs caused a frameshift in the puromcyin open reading frame (ORF), leading to a non-functional selection cassette. A site directed mutagenesis approach for correction was carried out. In this, the elongation factor 1 (EF1) promotor and the pruomycin ORF were amplified separately using primers that generated an overlapping sequence at the $3^{\prime}$ end of the EF1-fragment and the $5^{\prime}$ end of the puromycin fragment (Figure 3 A). Additionally, restriction cut sites for KspAl and Notl were inserted. PCR products were cleaned from the reaction mix and joined by PCR using $3^{\prime}$ and $5^{\prime}$ flanking primers. The frameshift EF1-puromycin fragment was cut from pAAVS1-CAG by digestion with KspAI and Notl. The corrected EF1-puro fragment was also digested with KspAl and Notl. Subsequently, the corrected EF1-puro fragment was ligated to PAAVS1-CAG and correct insertion verified by colony PCR, restriction digest and sequencing. In order to facilitate cloning of redox sensor constructs into the pAAVS1-CAG-EF1puro, a custom multiple cloning site (MCS) was inserted downstream of the CAG promotor (Figure 3 B). Oligonucleotides encoding the cutsite sequence for EcoRV-Sbfl-Agel-Pacl-Sall were supended in $10 \mathrm{mM}$ TRIS pH 8, $50 \mathrm{mM} \mathrm{NaCl}, 1 \mathrm{mM}$ EDTA at a final concentration of $40 \mathrm{nM}$ of each oligonucleotide and annealed by incubation at $95^{\circ} \mathrm{C}$ for $10 \mathrm{~min}$ in a metal rack and subsequently left to cool to room temperature. pAAVS1-CAG-EF1-puro was digested with Sall and ECoRV and ligated with MCS duplexes. Correct integration was verified by colony PCR, restriction digest and sequencing (pAAVS1MCS-EF1-puro).

Grx1-roGFP2 and mito-Grx1-roGFP2 coding sequences were amplified by PCR from pLPCX backbone vectors (a kind gift by Tobias Dick, Heidelberg) and restriction overhangs for Sall and Pacl inserted. After digestion of pAAVS1-MCS-EF1-puro and sensor fragments with Sall and Pacl, ligation was performed. Correct insert integration was verified by colony PCR, restriction digest and sequencing. Please refer to Appendix for detailed vector maps. 
A

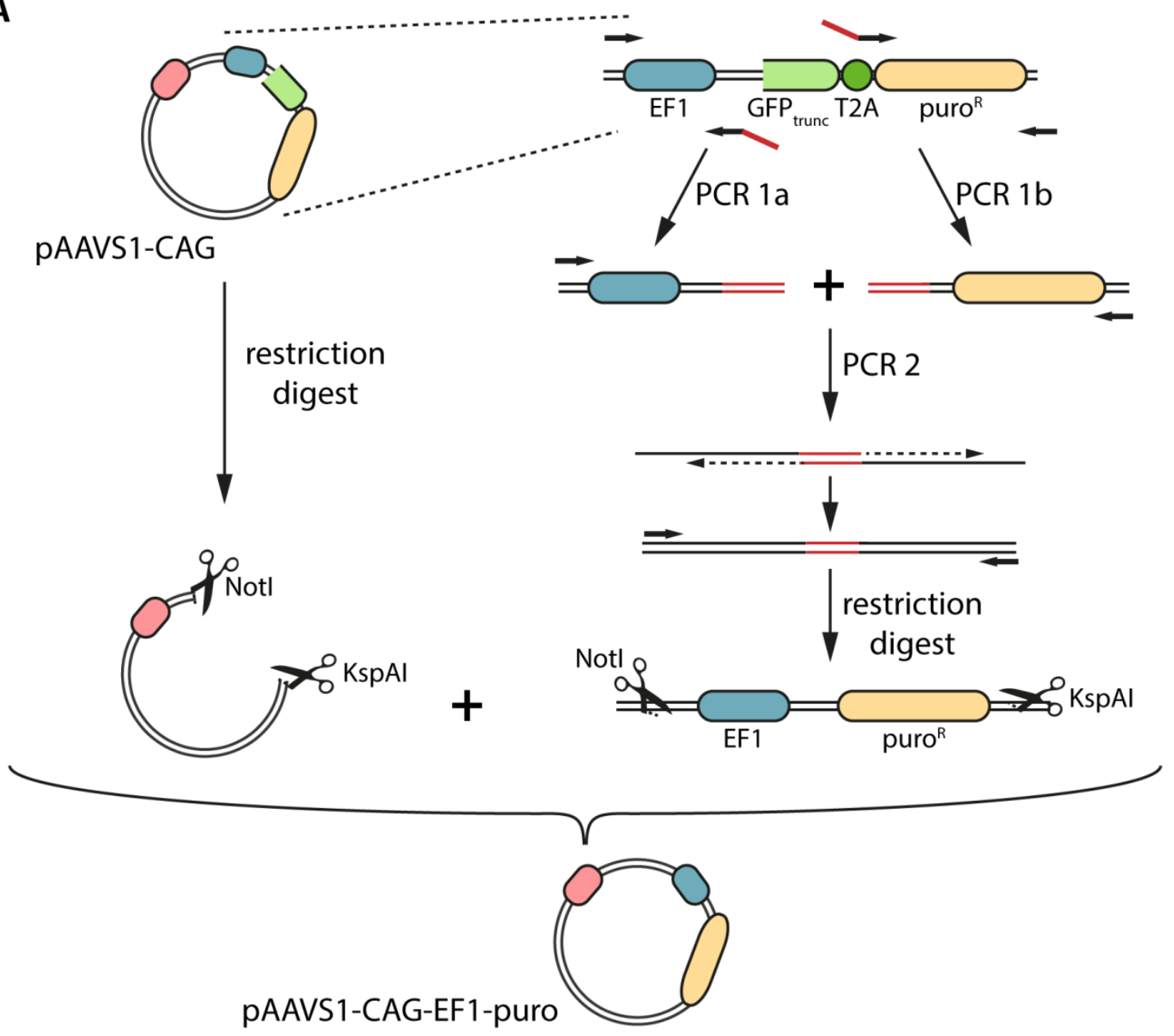

B

custom DNA oligos

3'-ATCCCTGCAGGACCGGTTTAATTAAG-5'

3'-TCGACTTAATTAAACCGGTCCTGCAGGGAT-5' oligo annealing

3'-ATCCCTGCAGGACCGGTTTAATTAAG-5'

ع

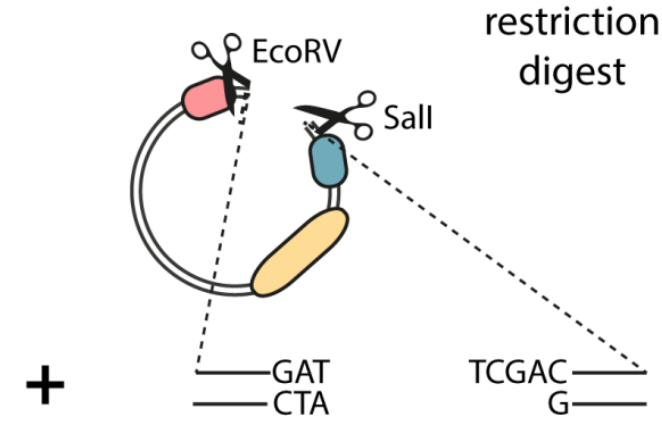

pAAVS1-CAG-MCS-EF1-puro

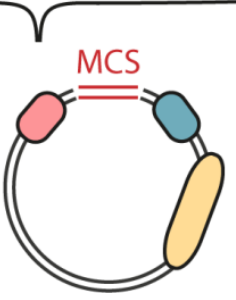

Figure 3 Schematic of cloning strategies. A) Correction of puromycin frameshift by mutagenesis PCR. B) Insertion of a custom MCS downstream of CAG promotor. 
HyPer and HyPerDAO coding sequences were amplified by PCR from a PAAVHy2DAAO-NLS plasmid (a kind gift by Vsevolod Belousov, Moscow) and restriction sites for EcoRV and Sall inserted. pAAVSCAG-MCS-EF1-puro and the sensor fragments were digested with EcoRV and Sall and ligated. Please refer to Appendix for detailed vector maps.

\subsection{2. pGIPZ lenti constructs}

pGIPZ-Grx1-roGFP2 for the expression of Grx1-roGFP2 was generated previously in our lab (Heta, 2017). A fragment encoding mito-Grx1-roGFP2 under the control of a cytomegalovirus (CMV) promotor was amplivied by PCR from pLPCX backbone vector (a kind gift by Tobias Dick, Heidelberg) and restriction overhangs for Xbal and Notl inserted. pGIPZ backbone vector and CMV-mito-Grx1roGFP2 were digested with Xbal and Notl, fragments run on a agarose gel, respective bands cut out and cleaned. Subsequent ligation resulted in pGIPZ-mito-Grx1-roGFP2. Correct insert integration was verified by colony PCR, restriction digest and sequencing. Please refer to Appendix for detailed vector maps.

\subsection{TALEN mediated integration}

\subsubsection{Linearization of donor vectors}

pAAVS1 donor vectors for sensor integration were linearized prior to TALEN co-transfection in order to facilitate homology directed repair. $20 \mu \mathrm{g}$ of plasmid DNA were digested with Scal in a $20 \mu \mathrm{l}$ reaction volume according to manufacturer's instructions. Linearized plasmids were cleaned using NucleoSpin Gel and PCR clean-up kit and eluted in $50 \mu \mathrm{l} \mathrm{H}_{2} \mathrm{O}$.

\subsubsection{Sensor integration in TSA cells}

TSA cells were grown on 6-well plates and transfected using PolyFect (Qiagen) according to manufacturer's instructions. Briefly, $2 \mu \mathrm{g}$ total DNA (1777 ng linearized donor plasmid, $111 \mathrm{ng}$ of each TALEN encoding vector) in $100 \mu \mathrm{l}$ supplement free medium were mixed with $20 \mu \mathrm{l}$ PolyFect and incubated at room temperature for $10 \mathrm{~min} .600 \mu \mathrm{l}$ complete growth medium were added to PolyFect-DNA Mix and transferred dropwise to cells. After $24 \mathrm{~h}$ recovery, selection with $1 \mathrm{\mu g} / \mathrm{ml}$ puromycin was initiated and increased to $2 \mu \mathrm{g} / \mathrm{ml}$ subsequently. Cells were split when confluency was reached and re-selected when $\mathrm{GFP}^{+}$content in live FC fell below $80 \%$.

\subsubsection{Sensor integration in pluripotent stem cells}

HES2 wild type cells were resuspended as described above (see 4.1.3) and electroporation carried out with the neon electroporator according to the manufacturer's instructions. Briefly, $1 * 10^{6}$ cells were pelleted and resuspended in $100 \mu$ lelectroporation buffer $\mathrm{R}$. The cell suspension was mixed with $5 \mu \mathrm{g}$ total plasmid DNA (4444 ng linearized donor plasmid, $270 \mathrm{ng}$ of each TALEN encoding 
vector) and electroporation was carried out using the $100 \mu$ neon tip and a single pulse at 1,200 V for 40 ms. Cells were immediately transferred onto fresh feeder plates with HES medium containing $5 \mu \mathrm{M}$ ROCK inhibitor Y27632. When cells reached confluency they were split onto 1:120 Matrige ${ }^{\mathrm{TM}}$ coated plates in E8 medium and puromycin selection was carried out with $0.2 \mu \mathrm{g} / \mathrm{ml}$ for five days.

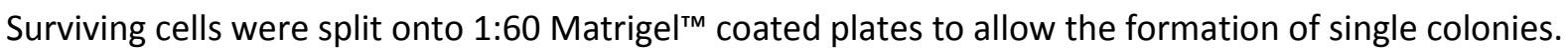
Fluorescent clones were marked and manually transferred onto a 96-well format. When individual clones reached confluency, they were consecutively transferred onto 48-well, 24-well and T25 culture formats. A sample for DNA isolation and genotyping was taken at the first splitting from the T25 culture format. At passage 7 after electroporation, cells were seeded onto irradiated feeder cells in HES medium.

\subsubsection{Genotyping}

Genomic integration of transgenes was analyzed by PCR based genotyping. Genomic DNA of TSA and HES2 cells was isolated with NucleoSpin Tissue Kit (macherey nagel) according to the manufacturer's instructions. Per sample $5 \mu \mathrm{l} 2 \mathrm{x}$ FastGene Polymerase mix, $0.2 \mu \mathrm{l}$ of each $10 \mu \mathrm{M}$ primer and up to $250 \mathrm{ng}$ template DNA were mixed and the volume adjusted to $10 \mu \mathrm{l}$ using bidest $\mathrm{H}_{2} \mathrm{O}$. Primers used are given in the Appendix (Table 7), the thermocycling protocol is given in Table 4. PCR products were analyzed on a 1\% TAE-agarose gel containing 1:2000 midori green DNA stain at $90 \mathrm{~V}$ for $60 \mathrm{~min}$.

Table 4 Thermocycling protocol for genotyping and colony PCR with FastGene polymerase

\begin{tabular}{cccc}
\hline & $\mathbf{T}\left({ }^{\circ} \mathbf{C}\right)$ & $\mathbf{t}$ ( $\mathbf{m m}: \mathbf{s s})$ \\
\hline initial denaturation & 95 & $03: 00$ \\
35 cycles & denature & 95 & $00: 30$ \\
& anneal & $\mathrm{Tm}$ & $00: 30$ \\
extend & 72 & $01: 00 / \mathrm{kb}$ \\
final extension & 72 & $01: 00 / \mathrm{kb}$ \\
hold & 4 & $\infty$ \\
\hline
\end{tabular}

\subsubsection{Transgene localization}

Transgene localization in HES2 was analyzed using the Universal GenomeWalker 2.0 kit (takara bio) according to the manufacturer's instructions. PCR products obtained from secondary PCR were analyzed by Sanger sequencing (seqlab) and sequences aligned to donor vectors sequences with SerialCloner 3.0 and human genome sequences using BLAST (NCBI). 


\subsection{Lentiviral sensor expression}

\subsubsection{Production of lentivirus}

TSA cells were seeded on $10 \mathrm{~cm}$ culture dishes and transfected with $3 \mu \mathrm{g}$ packaging plasmid pPAX, $3 \mu \mathrm{g}$ envelop plasmid pMD2 and $2 \mu \mathrm{g}$ expression plasmid pGIPZ-Grx1-roGFP2 or pGIPZ-mito-Grx1roGFP2 using Polyfect (Qiagen) according to manufacturer's instructions. Medium supernatant was harvested after $72 \mathrm{~h}$ and viral titer tested with Lenti-X GoStix (clontech). Viral particles were concentrated using vivapure lentiSELECT 40 Purification and Concentration kit (Sartorius) according to manufacturer's instructions. Virus concentrate was either directly used to transduce cells or aliquoted and stored at $-80^{\circ} \mathrm{C}$.

\subsubsection{Transduction of $\mathrm{hCM}$ and HFF}

For transduction of hCM or HFF, normal culture medium was supplemented with $2.5 \mu \mathrm{l} / \mathrm{ml}$ viral concentrate and $8 \mu \mathrm{g} / \mathrm{ml}$ polybrene. Cells were incubated for $48 \mathrm{~h}$ before the first medium change. After several media changes, viral titer was tested using the Lenti-X p24 Rapid Titer Kit (clontech) according to manufacturer's instructions. When viral titer was below $12.5 \mathrm{pg} / \mu \mathrm{l}$ cells were considered virus free and experiments performed.

\subsection{Flow cytometry}

For live cell flow cytometry, $1 * 10^{6}$ cells were resuspended in PBS supplemented with $5 \%$ FCS containing 1:100 Sytox Red Dead cell stain and incubated for $15 \mathrm{~min}$ at room temperature. Cells were then spun down at $300 \times \mathrm{xg}$ at $4^{\circ} \mathrm{C}$ for $4 \mathrm{~min}$ and resuspended in $300 \mu \mathrm{l}$ of PBS for analysis. Dead cells were excluded from analysis based on Sytox staining. Fluorescence gates were set according to non-fluorescent control cells.

Cell fixation for flow cytometry was carried out by incubation of $0.5-1 * 10^{6}$ cells in 2\% paraformaldehyde (PFA) for $15 \mathrm{~min}$ at room temperature. After washing with PBS cells were resuspended in PBS and stored at $4^{\circ} \mathrm{C}$ until analysis. After 5 min of blocking and permeabilization with blocking buffer (PBS, 10\% bovine serum albumin (BSA), 5\% FCS, 0.5\% Triton X-100), primary antibody staining was performed in blocking buffer at $4^{\circ} \mathrm{C}$ for $60 \mathrm{~min}$. After three washes with blocking buffer, secondary antibody staining was performed together with Hoechst DNA staining at $4^{\circ} \mathrm{C}$ for $45 \mathrm{~min}$. Samples were washed twice with blocking buffer, then once with PBS and resuspended in PBS for flow cytometry analysis. For each sample a negative control was incubated only with secondary antibody and Hoechst. If directly labelled antibodies were used, Hoechst staining was done in parallel with primary antibody incubation. Single, intact cells were gated based on forward scatter (FSC) vs sideward scatter (SSC) and doublets excluded based on Hoechst DNA 
stain. Gating was performed according to negative control samples. Analysis was performed with BD FACS Diva and Flowing2 software. All antibodies and dilutions used can be found in the Appendix (Table 6).

\subsection{Immunofluorescence}

For immunofluorescence staining, cells were grown on glass coverslips under growth conditions stated above. Cells were fixed with $4 \%$ PFA for $15 \mathrm{~min}$ at room temperature and stored in PBS at $4^{\circ} \mathrm{C}$ until staining. After one hour blocking with blocking buffer at room temperature (PBS, 10\% BSA, $5 \% \mathrm{FCS}, 0.5 \%$ Triton $\mathrm{X}-100$ ), primary antibody incubation was carried out at $4^{\circ} \mathrm{C}$ overnight. Next day, cells were washed three times for 5 min with PBS and secondary antibody staining carried out for one hour at room temperature. Slides were washed three times with PBS for 5 min and embedded on glass slides using Mowiol or Fluoromount-G. For antibodies and dilutions used, see Appendix (Table 6). Images were acquired using a Zeiss LSM710 confocal microscope and Zen software. Images were analyzed using FIJI-ImageJ and adobe illustrator.

\subsection{Live imaging of mitochondria}

Live cells were stained for active mitochondria using tetramethylrhodamine methylester (TMRM) according to manufacturer's instructions. TMRM was reconstituted at $10 \mathrm{mM}$ in methanol and stored at $-20^{\circ} \mathrm{C}$ until use. Before use, TMRM was diluted to as final concentration of $50 \mathrm{nM}$ in cell culture medium. For nuclear co-staining, 1:1000 Hoechst was added to the staining medium. Cells seeded on coverslips were stained for $30 \mathrm{~min}$ at $37^{\circ} \mathrm{C}$ in a humidified incubator, washed twice and imaged in either medium or imaging buffer. Images were acquired on a Zeiss LSM710 confocal microscope using Zen software and analyzed with FIJI-ImageJ and adobe illustrator.

\subsection{Plate reader based redox assays}

TSA cells were seeded onto optical bottom 96-well plates (zellkontakt and Thermo Fischer) in growth conditions stated above at a density of $1 * 10^{5} / \mathrm{cm}^{2}$ in order to achieve confluency on the day of measurement. Redox compounds such as $\mathrm{H}_{2} \mathrm{O}_{2}$, DTT and D-alanine were prepared as 10x stocks in imaging buffer (114 mM NaCl, $5.4 \mathrm{mM} \mathrm{KCl}, 1 \mathrm{mM} \mathrm{MgCl}, 2 \mathrm{mM} \mathrm{CaCl} 2[1 \mathrm{mM} \mathrm{CaCl}$ for hCM], $10 \mathrm{mM}$ HEPES; $\mathrm{pH}$ 7.4) on a round bottom 96-well plate. Before measurement, cells were washed twice with PBS and incubated in imaging buffer at $37^{\circ} \mathrm{C}$. Plates were transferred to the $37^{\circ} \mathrm{C}$ heated plate reader (flex station3, molecular devices) and measurements performed. Wells were excited sequentially at $405 \mathrm{~nm}$ and $488 \mathrm{~nm}$ and emission detected at $520 \mathrm{~nm}$ using a $515 \mathrm{~nm}$ cutoff filter. Fluorescence intensities were recorded every five second for a total of five minutes. At $60 \mathrm{~s}, 20 \mu \mathrm{l}$ compound solution were automatically added and mixed by trituration. Raw data were analyzed using Microsoft excel and graph pad prism. Background fluorescence values from empty wells were 
subtracted and the $405 \mathrm{~nm} / 488 \mathrm{~nm}$ ratio calculated. All ratios were normalized to the average of the respective baseline trace.

\subsection{Redox imaging}

Transgenic cells were seeded on $25 \mathrm{~mm}$ diameter glass coverslips using growth conditions stated above. Redox imaging was performed in imaging buffer $(114 \mathrm{mM} \mathrm{NaCl}, 5.4 \mathrm{mM} \mathrm{KCl}, 1 \mathrm{mM} \mathrm{MgCl}$, $2 \mathrm{mM} \mathrm{CaCl}$ [1 mM CaCl 2 for hCM], $10 \mathrm{mM} \mathrm{HEPES}$; $\mathrm{pH}$ 7.4) in a $37^{\circ} \mathrm{C}$ climate chamber on a Zeiss D1 Observer microscope using ZEN image processing software (Zeiss). Images were acquired sequentially at $400 \mathrm{~nm}$ and $505 \mathrm{~nm}$ excitation using a YFP filter at $40 \mathrm{x}$ magnification every $3 \mathrm{~s} . \mathrm{H}_{2} \mathrm{O}_{2}$ or DTT were added after a stable baseline was reached at $30 \mathrm{~s}$. Image analysis was carried out using FIJI-ImageJ with the Biovoxxel Toolbox, Python3.6 and GraphPad prism 7. Regions of interest (ROIs) were drawn by hand based on bright field and GFP images to correspond to single cells, as well as a background ROI not containing any cell per slide. Fluorescence intensities for each ROI in each channel were extracted and corresponding background values subtracted. The ratio of the corrected intensity values was normalized to the average of the baseline. Calculation of GSH redox potential $\left(\mathrm{E}_{\mathrm{GSH}}\right)$ was performed as previously described (Meyer \& Dick, 2010).

\subsection{Western Blot}

\subsubsection{Whole cell protein isolation}

For the preparation of whole cell protein lysates, transgenic cells were seeded on 6-well plates. When cells reached confluency, lysis was carried out by incubation with $100 \mu$ l Bäuerle buffer (20 mM HEPES, $350 \mathrm{mM} \mathrm{NaCl}, 1 \mathrm{mM} \mathrm{MgCl}$, 0.5 mM EDTA, $0.1 \mathrm{mM}$ EGTA, 1\% NP40, 20\% glycerol, pH 7.9) with Complete mini proteinase inhibitor and PhosphoStop phosphatase inhibitor (both Roche) on ice. After scraping culture surface with cell scraper, cell lysates were incubated for $20 \mathrm{~min}$ at $4^{\circ} \mathrm{C}$ while shaking. Further homogenization was achieved by $20 \mathrm{G}$ syringe. Lysates were centrifuged for $20 \mathrm{~min}$ at $10,000 \mathrm{xg}$ at $4^{\circ} \mathrm{C}$ and supernatants collected. Protein concentration was determined by Bradford assay and samples stored at $-20^{\circ} \mathrm{C}$ until use.

\subsubsection{Fractionation of mitochondria and cytosol}

Mitochondria isolation was carried out according to published protocol (Acín-Pérez et al., 2008), with modifications noted below. Cells were dissociated as described above, counted and $30-40 * 10^{6}$ cells pelleted and washed with PBS. Pellets were frozen at $-80^{\circ} \mathrm{C}$ to weaken the cell membrane and resuspended in $2 \mathrm{ml} 83 \mathrm{mM}$ sucrose, $10 \mathrm{mM}$ HEPES, pH 7.2. Cell suspension was transferred into a $2 \mathrm{ml}$ dounce homogenizer and homogenized using 30 strokes with a tight fitting pestle. An equal volume of $250 \mathrm{mM}$ sucrose, $30 \mathrm{mM}$ HEPES, pH 7.2 supplemented with $2 x$ complete mini protease 
inhibitor (Roche) were added and centrifuged at $1000 \mathrm{xg}$ for $5 \mathrm{~min}$ at $4^{\circ} \mathrm{C}$. Supernatants were transferred to clean microcentrifuge tubes and mitochondria pelleted at $12000 \mathrm{xg}$ for $15 \mathrm{~min}$ at $4^{\circ} \mathrm{C}$. Supernatants were retrieved as cytosolic fraction and mitochondria pellet resuspended in $200 \mu \mathrm{l}$ $320 \mathrm{mM}$ sucrose, $1 \mathrm{mM}$ EDTA, $10 \mathrm{mM}$ Tris- $\mathrm{HCl}$, pH 7.4. Protein content was quantified by Bradford assay and samples stored at $-80^{\circ} \mathrm{C}$ until use.

\subsubsection{Bradford assay}

Protein samples were diluted $1: 25$ in $\mathrm{H}_{2} \mathrm{O}$ and prepared in triplicates. A standard curve with concentrations ranging from $4 \mathrm{ng} / \mathrm{ml}$ to $64 \mathrm{ng} / \mathrm{ml} \mathrm{BSA}$ in $\mathrm{H}_{2} \mathrm{O}$ was prepared in duplicate. To each $50 \mu \mathrm{l}$ of protein solution, $200 \mu \mathrm{l}$ of $1 \times$ Rotiquant solution (Roth) in $\mathrm{H}_{2} \mathrm{O}$ were added. After $5 \mathrm{~min}$ incubation at room temperature, $\mathrm{OD}_{595}$ was measured with flexstation3 (molecular devices). Protein concentrations were extrapolated from standard curve after subtraction of background signal.

\subsubsection{SDS-polyacrylamid gel electrophoresis and western blot}

$35 \mu \mathrm{g}$ total protein per sample were prepared with $4 x$ Laemmli buffer $(0.12 \mathrm{M}$ Tris $-\mathrm{HCl}$, 0.025\% Bromophenol blue, 8\% SDS, 33.3\% glycerol, 10\% $\beta$-mercaptoethanol, pH 6.8) and incubated at $95^{\circ} \mathrm{C}$ for $5 \mathrm{~min} .12 \%$ SDS-polyacrylamide gels with $4 \%$ stacking gel were prepared according to Table 5. Samples were loaded onto $12 \%$ SDS-polyacrylamid gels in running buffer ( $25 \mathrm{mM}$ Tris base, $200 \mathrm{mM}$ glycine, $3 \mathrm{mM} \mathrm{SDS}$ ) and run at $70 \mathrm{~V}$ until samples reached separating gel. Voltage was then increased to $120 \mathrm{~V}$ and gel run for $1-1.5 \mathrm{~h}$ until loading dye was running out. Proteins were transferred by semidry blotting onto polyvinylidene difluoride (PVDF) membrane using transfer buffer (62.2 mM Tris base, $10 \mathrm{mM}$ glycine, $5 \mathrm{mM}$ SDS, 20\% methanol) and blotting for $1 \mathrm{~h}$ at $140 \mathrm{~mA}$. Successful transfer was confirmed by staining with $0.1 \%$ Ponceau in $0.5 \%$ acetic acid, and membranes subsequently blocked with $5 \%$ non-fat milk powder in Tris-buffered saline with Tween (TBS-T; $20 \mathrm{mM}$ Tris- $\mathrm{HCl}, 165 \mathrm{mM} \mathrm{NaCl}, 0.1 \%$ Tween-20, pH 7.6). Primary antibody incubation in $5 \%$ non-fat milk powder in TBS-T was carried out at $4^{\circ} \mathrm{C}$ over night. After 3 washes with TBS-T for $10 \mathrm{~min}$, secondary HRP labelled antibody was incubated for $1 \mathrm{~h}$ at room temperature in $5 \%$ non-fat milk powder in TBS-T. Membranes were washed 3 times for $10 \mathrm{~min}$ with TBS-T. Antibody signal was detected using FemtoLucent HRP (G biosciences) according to manufacturer's instructions on a GelDoc XR imager (BioRad). If reprobing with another primary antibody was to be performed, membranes were stripped by two times 10 min incubation with stripping buffer ( $200 \mathrm{mM}$ glycine, $3.5 \mathrm{mM}$ SDS, 1\% Tween-20, pH 2.2), followed by three washes for $10 \mathrm{~min}$ with TBS-T. Afterwards, membranes were blocked and incubated as described above. For a list of antibodies and dilutions used, please refer to Appendix (Table 6). 
Table 5 Preparation of SDS-polyacrylamid gels

\begin{tabular}{lcccc}
\hline & \multicolumn{2}{c}{ 4\% stacking gel } & \multicolumn{2}{c}{ 12\% separating gel } \\
\hline & $\begin{array}{c}\mathrm{V}(\mathrm{ml}) \text { for } \\
2 \text { gels }\end{array}$ & $\begin{array}{c}\text { final } \\
\text { concentration }\end{array}$ & $\begin{array}{c}\mathrm{V}(\mathrm{ml}) \text { for } \\
\text { 2 gels }\end{array}$ & $\begin{array}{c}\text { final } \\
\text { concentration }\end{array}$ \\
\hline 40\% Acrylamide & 0.5 & $4 \%$ & 3 & $12 \%$ \\
$0.5 \mathrm{M}$ Tris pH 6.8 & 1.25 & $125 \mathrm{mM}$ & & \\
$1.5 \mathrm{M}$ Tris pH 8.8 & & & 2,5 & $375 \mathrm{mM}$ \\
$10 \%$ SDS & 0,05 & $0.10 \%$ & 0,1 & $0.10 \%$ \\
$10 \%$ APS & 0,05 & $0.10 \%$ & 0,1 & $0.10 \%$ \\
TEMED & 0,005 & $0.01 \%$ & 0,004 & $0.004 \%$ \\
bidest. Water & ad $5 \mathrm{ml}$ & & ad $10 \mathrm{ml}$ & \\
\hline
\end{tabular}




\section{Results}

\subsection{The GSH/GSSG redox milieu in subcellular compartments}

The GSH/GSSG system is the main cellular buffer system for ROS. Disturbances in redox homeostasis are believed to play an important role in the initiation and progression of cardiac diseases. The aim of the thesis was to develop a transgenic reporter system to optically assess the GSH/GSSG balance in human heart muscle. We therefore tested the GSH sensor Grx1-roGFP2 and a mitochondria targeted variant in TSA cells before exploring their applicability in undifferentiated HES2 and HES2 derived cardiomyocytes.

\subsubsection{Grx1-roGFP2 can be targeted to specific compartments in TSA cells}

We generated expression plasmids for the genetic integration of GSH sensor Grx1-roGFP2 and its mitochondria targeted variant, mito-Grx1-roGFP2. Mitochondrial targeting is achieved through a signal peptide sequence from bacterial Atp9, an ATP synthase. In order to test the correct expression and localization of the sensors, TSA cells were transfected with linearized donor vectors and TALEN expression plasmids, targeting the AAVS1 locus for integration. Subsequent puromycin selection ensured the enrichment of cells with successful genetic integration (Figure 4 A). Co-staining with mitochondria marker TMRM revealed a co-localization with the GFP signal in mito-Grx1-roGFP2 cells. GFP fluorescence from untargeted Grx1-roGFP2 was distributed across the whole cell, including cytoplasm and nucleus. The localization of sensor to the two compartments was further corroborated by Western blot anaylsis of cellular fractions. Cytosolic and mitochondria protein fractions were plotted and membranes stained for GFP, respiratory chain components and glycerolaldehydephosphatedehydrogenase (GAPDH). Respiratory chain proteins were only detectable in mitochondria fractions, whereas GAPDH was enriched in cytosolic fractions (Figure 4 B). For Grx1-roGFP2 cells, GFP was enriched in the cytosolic fraction, whereas mito-Grx1-roGFP2 showed a strongly enriched GFP signal in the mitochondrial fraction. In both cases, a small amount of GFP was also detected in the other fraction, possibly due to the crude preparation of mitochondria. 
A

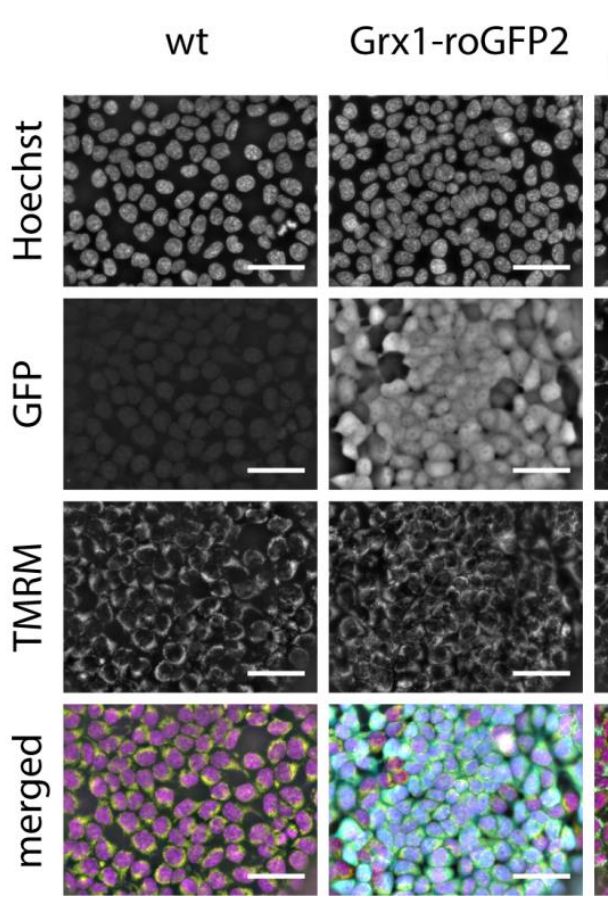

mito-
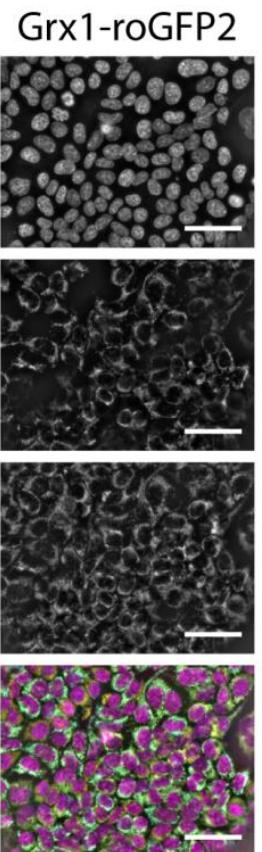

B

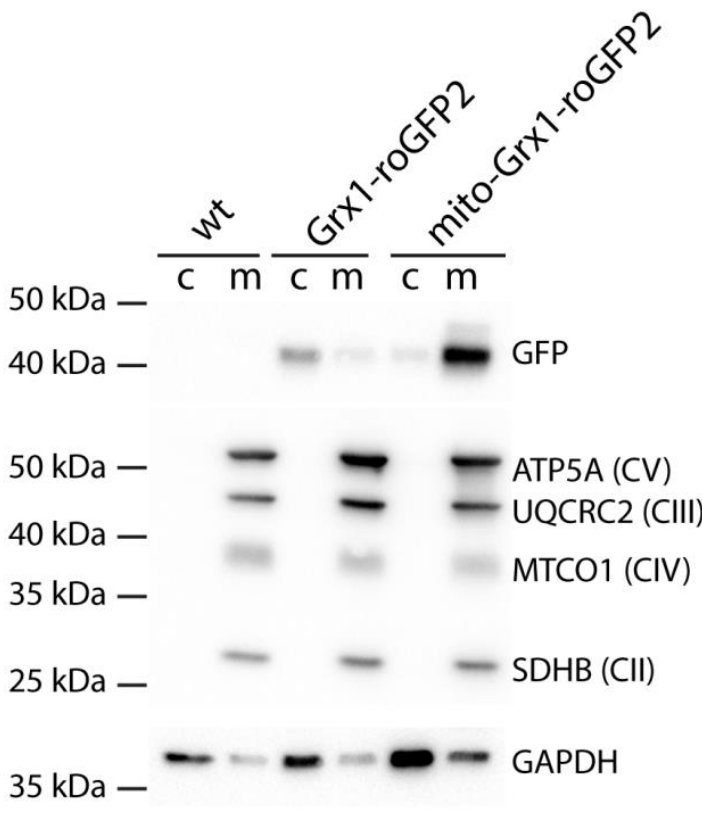

Figure 4 Grx1-roGFP2 is expressed in a compartment specific manner in TSA cells. TSA cells were transfecte with donor vectors for either untargeted (Grx1-roGFP2) or mitochondria trageted (mito-Grx1-roGFP2) redox sensors. A) Live fluorescence imaging of GFP, nuclei (Hoechst) and mitochondria (TMRM) demonstrates successful targeting of Grx1roGFP2 to cytosol and mitochondria. Scale bars: $50 \mu \mathrm{m}$. B) Western blot of cytosolic (c) and mitochondria (m) protein fractions confirms sensor enrichment in the targeted fraction. Purity of fractionation is demontrated by respiratory chain complexes I-IV in mitochondria fractions (SDHB: succinate dehydrogenase complex iron sulfur subunit B, MTCO1: Cytochrome c oxidase I, UQCRC2: ubiquinol-cytochrome c reductase core protein II, ATP5A: ATP synthase subunit alpha). Figure and legend as published in (Trautsch et al., 2019).

\subsubsection{Plate reader based redox assays confirm function of Grx1-roGFP2 in TSA cells}

Upon oxidation the maximum excitation wavelength of the fluorescent redox sensor Grx1-roGFP2 is shifted from $488 \mathrm{~nm}$ to $405 \mathrm{~nm}$. To test the function of Grx1-roGFP2 in TSA cells, we performed plate reader based redox assays. To this end, TSA cells were seeded on optical bottom 96-well plates and fluorescence intensity was measured upon excitation with $405 \mathrm{~nm}$ and $488 \mathrm{~nm}$. An automated liquid handling system integrated in the plate reader was used to deliver redox active compounds such as $\mathrm{H}_{2} \mathrm{O}_{2}$ or DTT after baseline recording. In this assay, we were able to detect a concentration dependent increase of fluorescence intensity ratio $R_{(405 / 488)}$ upon oxidation for both cytosolic and mitochondria targeted sensors. In both cases, a concentration of $1 \mu \mathrm{M} \mathrm{H}_{2} \mathrm{O}_{2}$ was not sufficient to induce detectable sensor oxidation. In the cytosol, a maximum oxidized ratio of $4.190 \pm 0.336$, $\mathrm{n}=11$, was reached upon application of $100 \mu \mathrm{M} \mathrm{H}_{2} \mathrm{O}_{2}$ (Figure $5 \mathrm{~A}$ ), whereas mitochondria appeared to be already maximally oxidized at $10 \mu \mathrm{M} \mathrm{H}_{2} \mathrm{O}_{2}$, with a maximum oxidized ratio of $2.806 \pm 0.291$, $\mathrm{n}=7$, upon application of $100 \mu \mathrm{M} \mathrm{H}_{2} \mathrm{O}_{2}$ (Figure $5 \mathrm{~B}$ ). DTT application of concentrations up to $1 \mathrm{mM}$ did not lead to visible reduction of the cytosol (minimum reduced ratio $0.817 \pm 0.047, n=12$; Figure 5 C). Mitochondria, however, demonstrated a reduction down to $0.485 \pm 0.106, n=6$, minimum 
reduced ratio when treated with $1 \mathrm{mM}$ DTT (Figure $5 \mathrm{D}$ ). Taken together, these results indicate a higher oxidation capacity in cytosol compared to mitochondria, i.e. a more oxidized GSH pool in mitochondria of TSA cells.

A

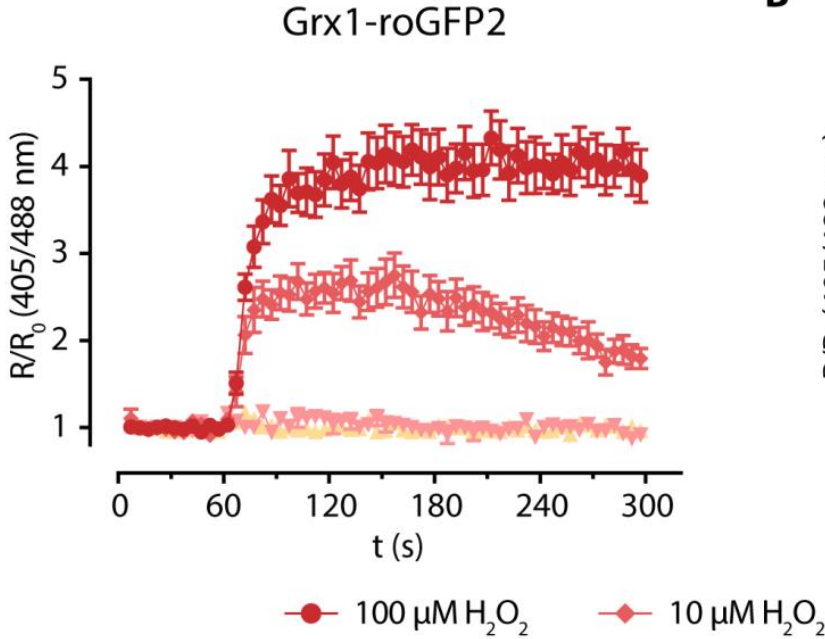

B

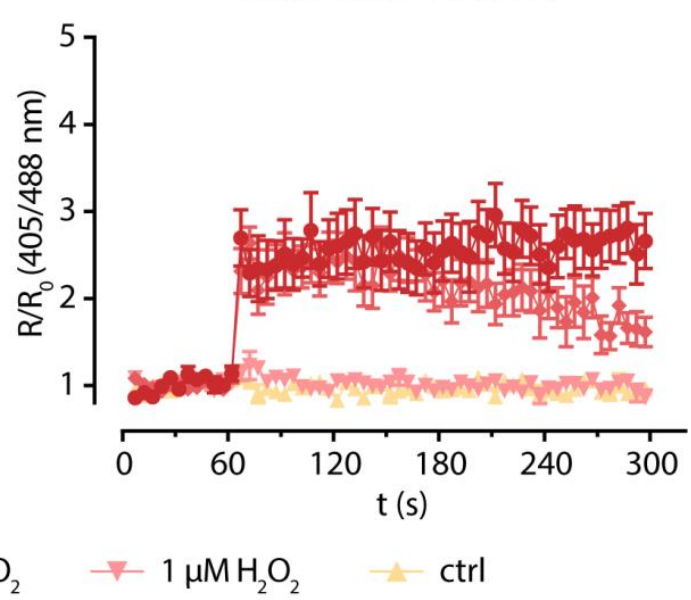

C
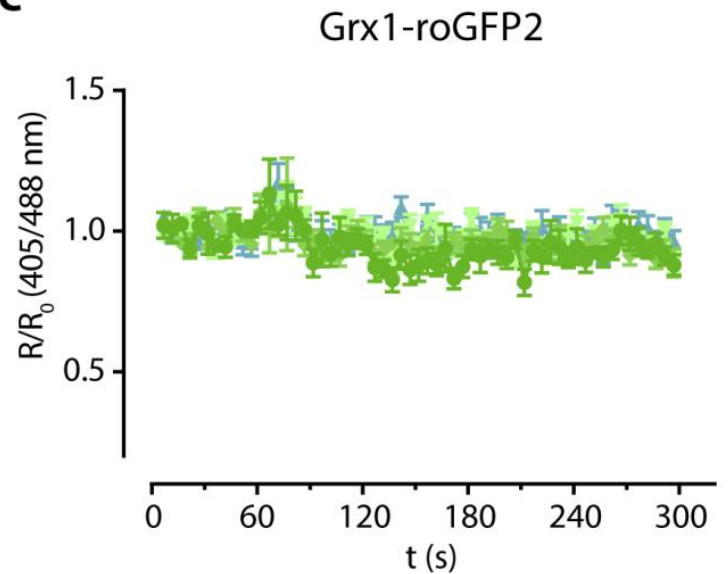

D
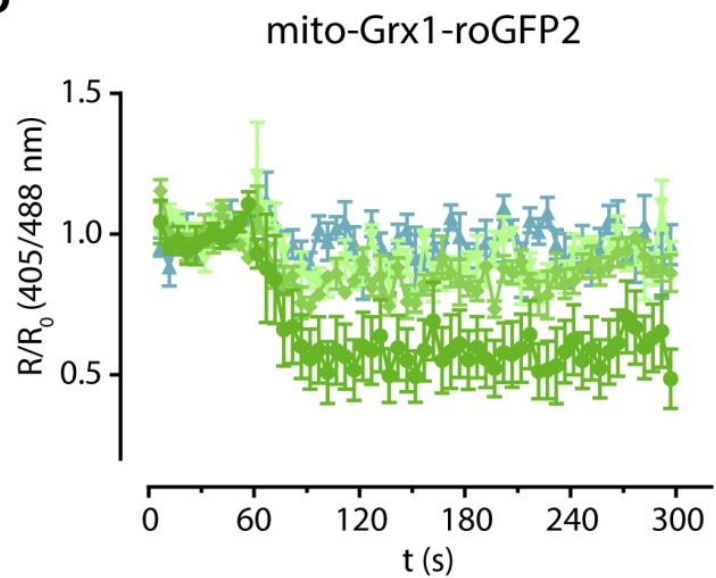

$-10 \mu \mathrm{MDTT} \rightarrow \mathrm{ctrl}$

Figure 5 Grx1-roGFP2 expressed in cytosol or mitochondria of TSA cells reacts to oxidation and reduction. Fluorescence intensity upon excitation with $405 \mathrm{~nm}$ and $488 \mathrm{~nm}$ was recorded every $5 \mathrm{~s}$ and is displayed as the change of fluorescence ratio $(R)$ over baseline $\left(R_{0}\right)$ as a function of time. TSA cells treated with indicated concentrations of $\mathrm{H}_{2} \mathrm{O}_{2}$ and DTT at $60 \mathrm{~s}$. A, C) cytosolic localized sensor GRX1-roGFP2, B, D) mitochondria targeted sensor mito-Grx1-roGFP2. Error bars: SEM, $n=4-12$.

\subsubsection{Genomic integration leads to redox sensor expression in HES2}

We aimed to integrate fluorescent redox sensors Grx1-roGFP2 and mito-Grx1-roGFP2 into the safe harbor AAVS1 locus on chromosome 19 of human embryonic stem cells. HES2 were electroporated with linearized donor vectors encoding for a redox sensor and a puromycin selection marker. In addition two plasmids encoding a TALEN system targeting the AAVS1 locus were co-electroporated. GFP positive clones were selected and expanded. Two lines, HES2 cyto\#3, expressing Grx1-roGFP2 and HES2 mito2\#34, expressing mito-Grx1-roGFP2 were selected for further analysis based on their 
fluorescence properties. Analysis of pluripotency marker expression by flow cytometry for Oct4, Nanog and Tra1-60 revealed no differences between transgenic lines and non-transgenic controls (Figure 6 A). PCR of genomic DNA demonstrated integration of sensor coding sequences in both lines (Figure $6 \mathrm{~B}, \mathrm{C}$ ). In conclusion, the integration and expression of fluorescent redox sensor did not influence pluripotency of the analyzed HES2 lines.

A

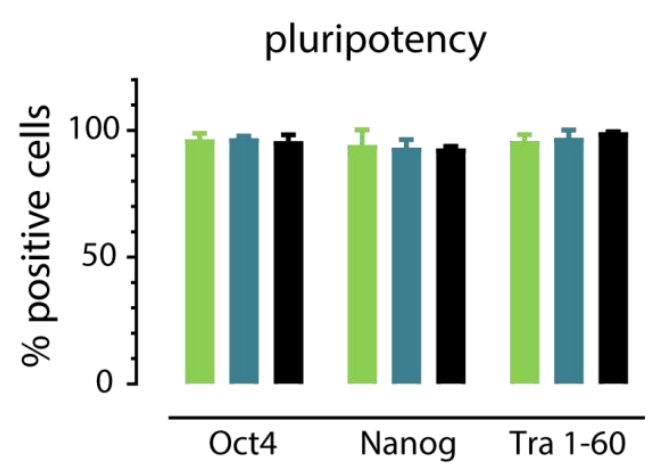

C

HES2 cyto\#3 HES2 mito3\#34 HES2 wt

B

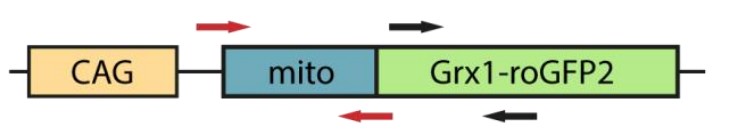

$400 \mathrm{bp}$ $300 \mathrm{bp}$ $200 \mathrm{bp}$

Figure 6 Transgene integration does not influence pluripotency. HES2cyto\#3 (expressing Grx1-roGFP2) and HES2mito2\#34 (expressing mito-Grx1-roGFP2) lines were obtained from electroporation of wild type HES2. A) After transgene integration, HES2 lines retain expression of pluripotency markers Oct4, Nanog, and Tra1-60. Flow cytometry analysis, error bars: SEM, data from $n=3$ passages. B) Schematic of duplex PCR for genotyping of transgenic lines. Two primer pairs were used in a single PCR reaction giving rise to one band for Grx1-roGFP2 (black arrows, $165 \mathrm{bp}$ ) and one band for mitochondrial targeting sequence (red arrows, 348 bp). C) Representative agarose gel electrophoresis for genotyping of HES2 lines. pAAVS1 donor vectors are used as positive control. Figure panel A adapted from (Trautsch et al., 2019).

We used a TALEN system to target integration of redox sensors to the AAVS1 safe harbor locus. Contrary to our expectations, we were not able to detect locus integration by PCR using primers overlapping genomic and transgene sequences. In order to evaluate where the sensor DNA was integrated, we performed a genome walking analysis. Genomic DNA from HES2 lines was digested using 3 different restriction enzymes, ligated with specific adapters and a nested PCR was performed (Figure 7 A). Agarose gel electrophoresis showed several bands for both lines and all three libraries analyzed. Six bands were cut out from HES2 cyto\#3 libraries and 5 bands from HES2 mito2\#34 libraries, DNA cleaned and sent for sequencing (Figure 7 B). DNA fragments from HES2 cyto\#3 bands 1 and 2 annealed to chromosome 16, syntaxin $1 \beta$ intron 1 (nucleotides 31009712-31009446, genome assembly GRCh38.p12), whereas the sequence recovered from HES2 mito2\#34 band 4 annealed to a promotor flank region on band p21 on chromosome 1 (nucleotides 4308490743085978). For all other DNA fragments analyzed, no sequencing results could be obtained due to 
low DNA amount and quality. These results confirmed a random integration of sensor transgenes in the analyzed HES2 lines. Integration into further loci cannot be excluded, as we did not obtain sequence information for all bands present.

A

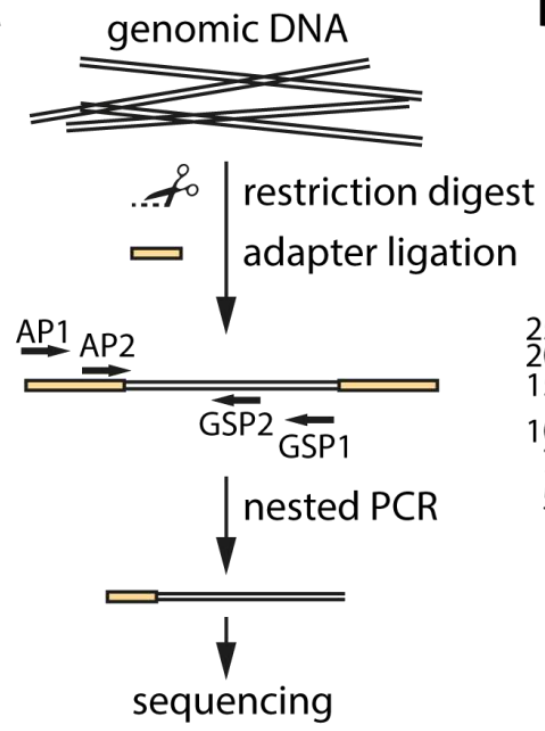

B

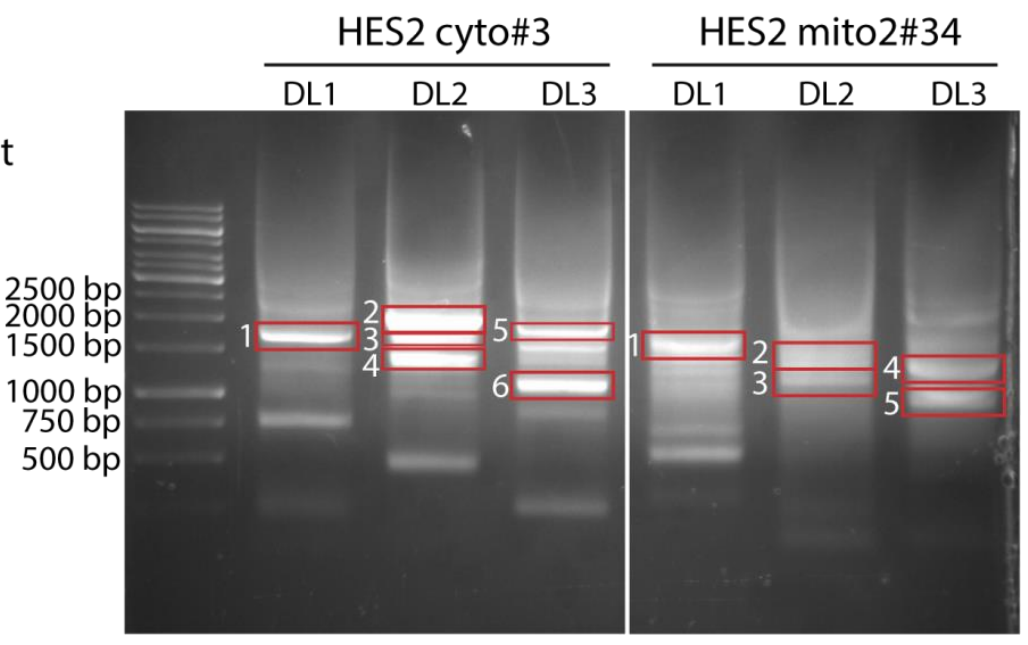

Figure 7 Redox sensor transgenes integrated at random sites in the HES2 genome. A) Principle of genome walker integration site analysis. B) Secondary (nested) PCR products separated on a $1 \%$ agarose gel. Bands that were isolated from the gel and sequenced are marked by red boxes and numbered. AP1/2: adapter primer 1/2, GSP1/2: gene specific primer 1/2, DL: DNA library.

We next analyzed the expression of the integrated transgenes. Live cell flow cytometry of HES2 cyto\#3 and HES2 mito2\#34 confirmed a uniform expression of sensor in both lines compared to wild type HES2 (Figure 8 A). Western blot of mitochondria and cytosol fractions confirmed a compartment enriched expression. In Grx1-roGFP2 expressing HES2 cyto\#3, GFP was enriched in the cytosolic fraction, whereas mito-Grx1-roGFP2 line HES2 mito2\#34 showed a strong GFP signal in the mitochondria fraction (Figure $8 \mathrm{~B}$ ). Since random integration did not seem to attenuate GFP expression in these cell lines and picked clones were uniformly positive for redox sensor expression, we continued to analyze the sensor function. 
A

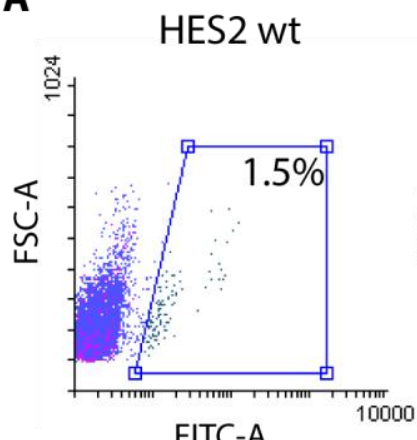

FITC-A

B

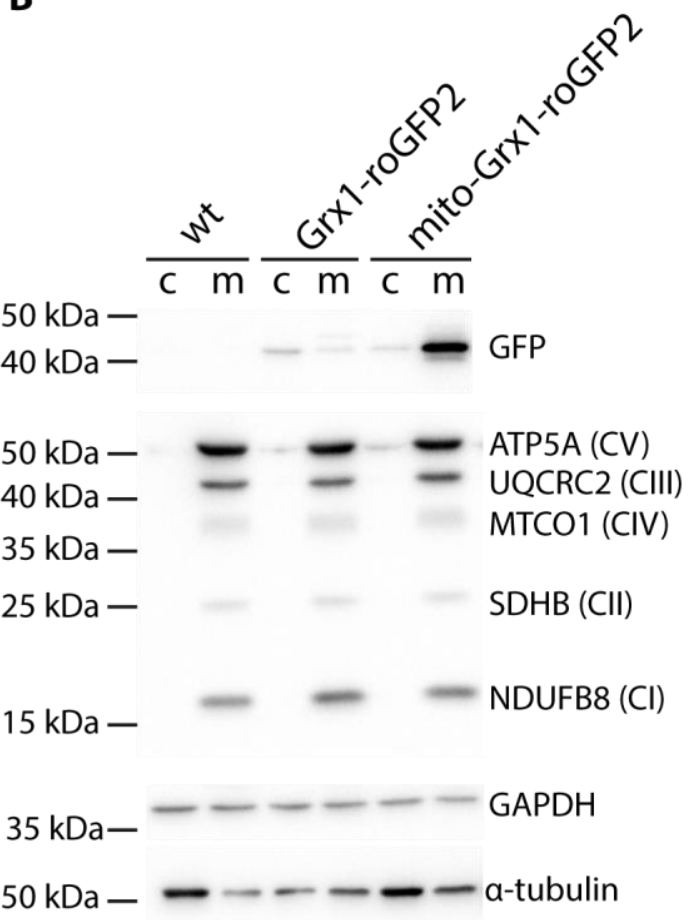

HES2 mito-Grx1-roGFP2

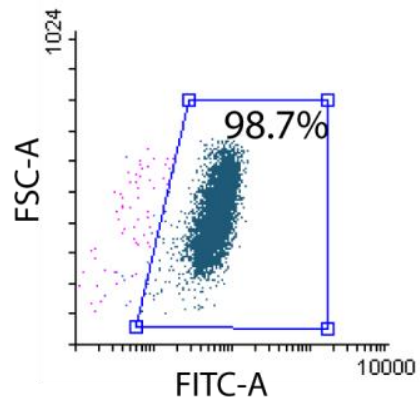

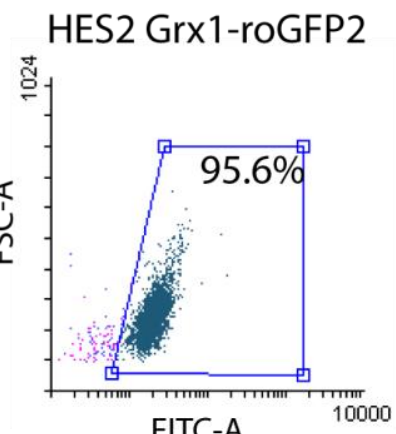

FITC-A

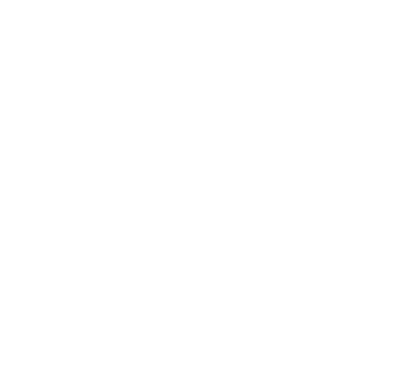

Figure 8 Grx1-roGFP is expressed in the targeted subcellular compartments in transgenic HES2. A) Live cell flow cytometry demonstrated uniform expression of fluorescent sensor in transgenic HES2. B) Western blot of cytosolic (c) and mitochondria $(\mathrm{m})$ fractions shows enrichment of GFP in either cytosol (HES2 cyto\#3) or mitochondria (HES2 mito2\#34). Purity of fractionation is demontrated by respiratory chain complexes I-IV in mitochondria fractions (NDUFB8: NADH ubiquinone oxidoreductase subunit B8, SDHB: succinate dehydrogenase complex iron sulfur subunit B, MTCO1: Cytochrome c oxidase I, UQCRC2: ubiquinol-cytochrome c reductase core protein II, ATP5A: ATP synthase subunit alpha). Figure and legend adapted from (Trautsch et al., 2019).

\subsubsection{Undifferentiated HES2 show a more reduced cytosol compared to mitochondria}

Undifferentiated HES2 cyto\#3 and HES2 mito2\#34 were subjected to redox imaging to test for the function of the integrated redox sensor transgenes. Cells were sequentially excited using $400 \mathrm{~nm}$ and $500 \mathrm{~nm}$ light and emission recorded with a YFP filter set. After baseline recording, oxidizing $\left(\mathrm{H}_{2} \mathrm{O}_{2}\right)$ or reducing (DTT) compounds were applied. We detected a concentration dependent increase in fluorescence ratio $\mathrm{R}_{(405 / 488)}$ upon oxidation with $\mathrm{H}_{2} \mathrm{O}_{2}$, while reduction with DTT led to a concentration dependent decrease in $\mathrm{R}_{(405 / 488)}$. In HES2 cyto\#3, oxidation with $300 \mu \mathrm{M} \mathrm{H}_{2} \mathrm{O}_{2}$ led to an increase of fluorescent ratio to $1.98 \pm 0.02, n=241$ (Figure $9 \mathrm{~A}$ ), whereas reduction induced a ratio decrease to $0.72 \pm 0.01, \mathrm{n}=197$, at $10 \mathrm{mM}$ DTT (Figure $9 \mathrm{C}$ ). Likewise, HES2 mito2\#34 reacted to $300 \mu \mathrm{M} \mathrm{H} \mathrm{O}_{2}$ with a maximum ratio increase to $1.78 \pm 0.01, \mathrm{n}=182$ (Figure $9 \mathrm{~B}$ ), and a minimum ratio decrease to $0.57 \pm 0.003, \mathrm{n}=193$, upon treatment with $10 \mathrm{mM}$ DTT (Figure $9 \mathrm{D}$ ). In both cellular compartments, cytosol and mitochondria, $1 \mu \mathrm{M} \mathrm{H}_{2} \mathrm{O}_{2}$ did not elicit a sensor response, while $10 \mu \mathrm{M}$ led to a moderate ratio increase. In mitochondria, $100 \mu \mathrm{M} \mathrm{H}_{2} \mathrm{O}_{2}$ were already able to induce maximal oxidation, while in cytosol, maximum oxidation was observed with $300 \mu \mathrm{M} \mathrm{H}_{2} \mathrm{O}_{2}$ only. Similarly, $10 \mu \mathrm{M}$ DTT did not lead to changes in $\mathrm{R}_{(405 / 488)}$ in neither cytosol nor mitochondria, while 
moderate reduction was observed after application of $100 \mu \mathrm{M}$ DTT. Concentrations of $1 \mathrm{mM}$ to $10 \mathrm{mM}$ DTT appeared to induce full reduction in both compartments. Treatment of HES2 mito2\#34 with $1 \mathrm{mM}$ DTT appeared to induce a stronger reduction response than treatment with $3 \mathrm{mM}$ DTT (Figure $9 \mathrm{D}$ ). This was not observed with HES2 cyto\#3 where reduction with DTT was concentration dependent, with $3 \mathrm{mM}$ DTT eliciting stronger response than $1 \mathrm{mM}$ (Figure $9 \mathrm{C}$ ). Further studies are needed to elucidate whether this difference in sensor response to DTT stimulation represents a physiological difference between the analyzed compartments.

A

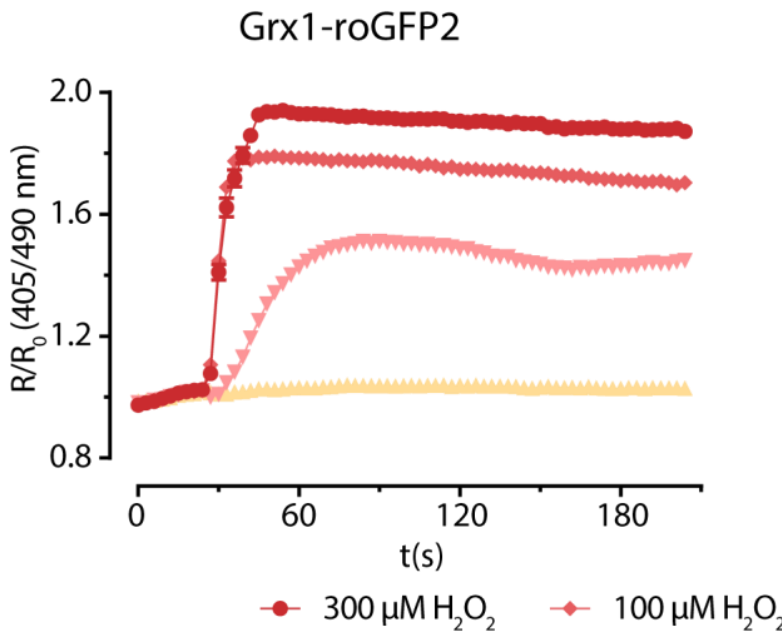

C

Grx1-roGFP2

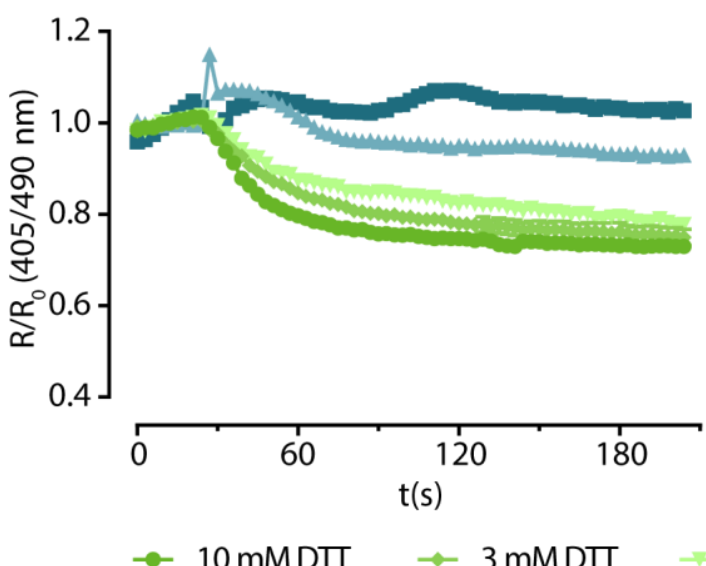

B mito-Grx1-roGFP2

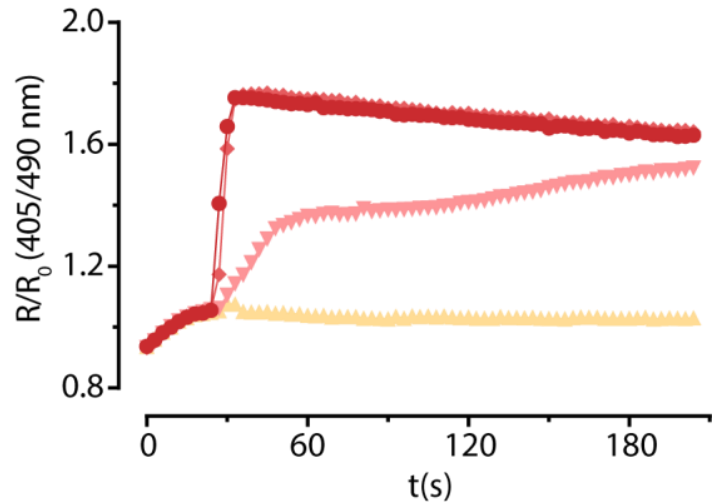

$1 \mu \mathrm{MH}_{2} \mathrm{O}_{2}$

D

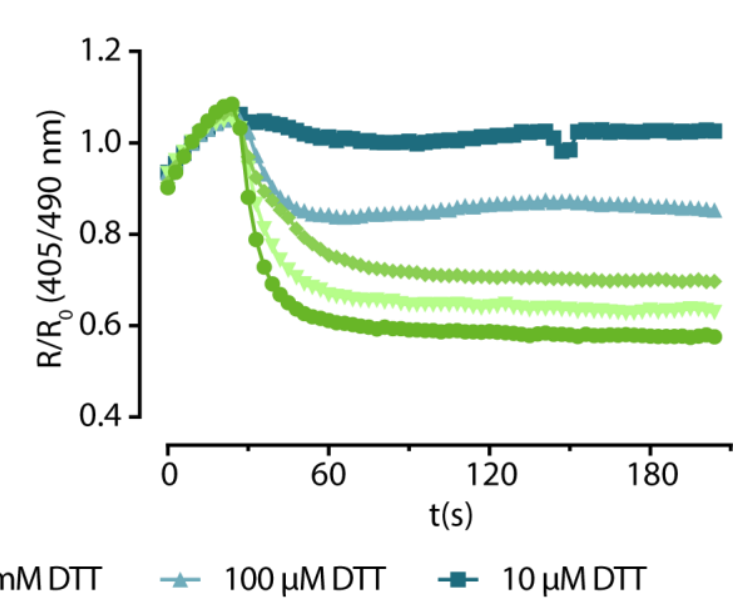

Figure 9 HES Grx1-roGFP2 and mito-Grx1-roGFP2 react to oxidation and reduction in a concentration dependent manner. Undifferentiated HES2 were subjected to redox imaging and fluorescence intensity upon excitation with $405 \mathrm{~nm}$ and $488 \mathrm{~nm}$ was recorded every $3 \mathrm{~s}$ and is displayed as the change of fluorescence ratio $(R)$ over baseline $\left(R_{0}\right)$ as a function of time. $\mathrm{H}_{2} \mathrm{O}_{2}$ or DTT were added at $30 \mathrm{~s}$ at the indicated concentrations. Error bars: SEM, $\mathrm{n}=52-274$. Figure and legend adapted from (Trautsch et al., 2019).

Based on maximal oxidation and reduction responses or Grx1-roGFP2, the redox potential for GSH/GSSG ( $\left.E_{G S H}\right)$ can be calculated using the Nernst equation (Meyer \& Dick, 2010). Based on our 
findings, cytosolic $E_{G S H}$ in undifferentiated stem cells was $-288.5 \mathrm{mV} \pm 1.5 \mathrm{mV} \quad(n=197)$. Mitochondrial $\mathrm{E}_{\mathrm{GSH}}$ was significantly more oxidized with $-277.3 \mathrm{mV} \pm 4.6 \mathrm{mV}(\mathrm{n}=182)$.

\subsubsection{HES2cyto\#3 and HES2 mito2\#34 derived cardiomyocytes are developmentally impaired}

Transgenic HES2 lines were differentiated to cardiomyocytes using a 21 day protocol. Differentiation efficiency was assessed by flow cytometry analysis of cardiac $\alpha$-actinin expression and GFP fluorescence. In HES2 cyto\#3 and HES2 mito2\#34 cardiomyocytes, $\alpha$-actinin positive cells were also GFP positive $(92.6 \% \pm 1.9 \%$ and $85.1 \% \pm 6.1 \%$ vs $0.1 \% \pm 0.02 \%$ and $0.2 \% \pm 0.1 \%$, respectively, $n=3$; Figure $10 \mathrm{~A}$ ). In non-transgenic control cardiomyocytes, no GFP positive cells were detected. Interestingly, differentiation efficiency was similar to transgenic sensor lines with $81.8 \% \pm 5.4 \%$, $n=3, \alpha$-actinin positive cells (Figure 10 A). Despite clear $\alpha$-actinin expression, we did not observe spontaneous beating activity in sensor line derived cardiomyocytes in 10 out of 12 differentiations. Immunofluorescence analysis of $\alpha$-actinin revealed a sarcomeric phenotype with less ordered sarcomeres and less strong striations in HES2 cyto\#3 and HES2 mito2\#34 compared to nontransgenic controls (Figure $10 \mathrm{~B}$ ). This functional phenotype was unexpected, as undifferentiated HES2 did not show any impairment of pluripotency. Furthermore, the identified integration sites where not associated with known cardiac genes (see also 5.1.3). Thus the data suggest a role for ROS in cardiomyocyte maturation and sarcomere assembly and a deleterious ROS buffering effect by constitutive Grx1-roGFP2 sensor expression. 
A

differentiation efficiency

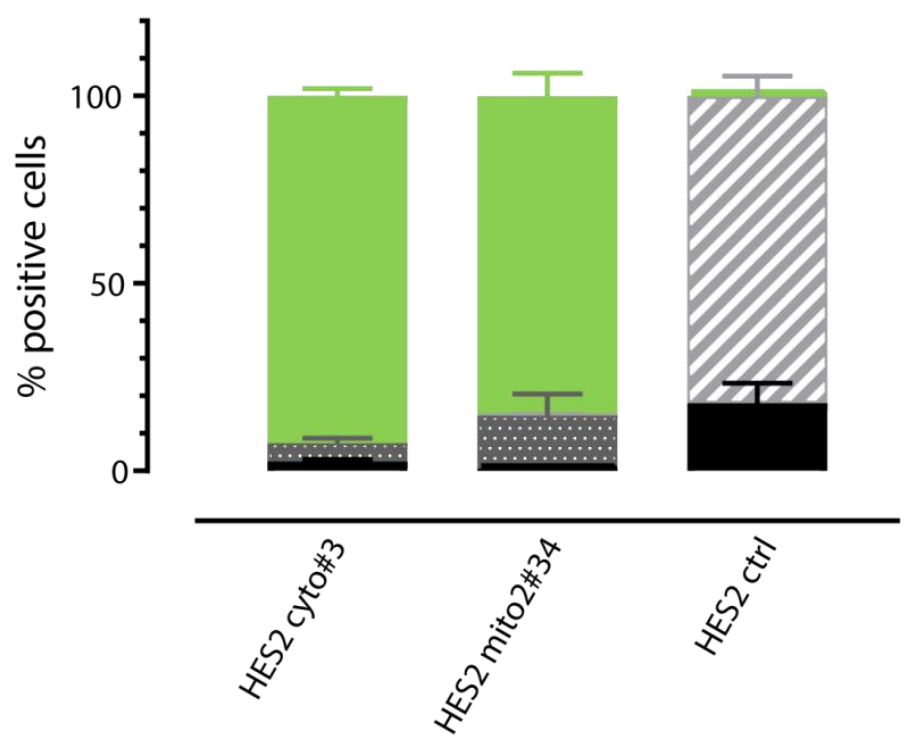

GFP $^{+}$actinin $^{+}$

02 GFP- $^{-}$actinin $^{+}$

2.... $\mathrm{GFP}^{+}$actinin $^{-}$

GFP- actinin

B
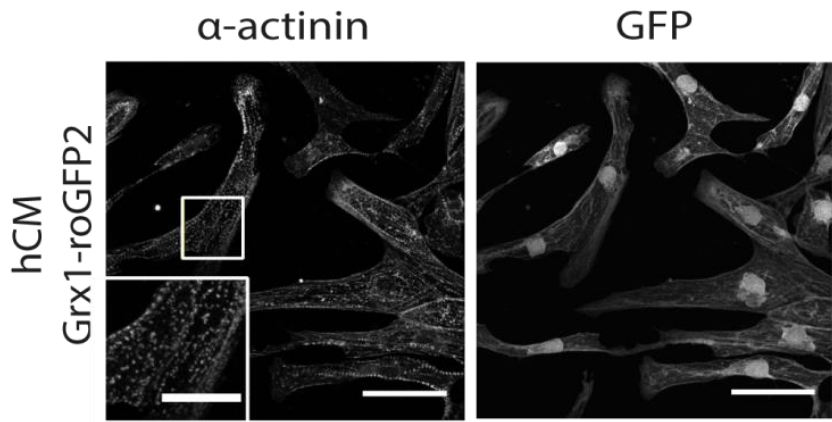

Hoechst
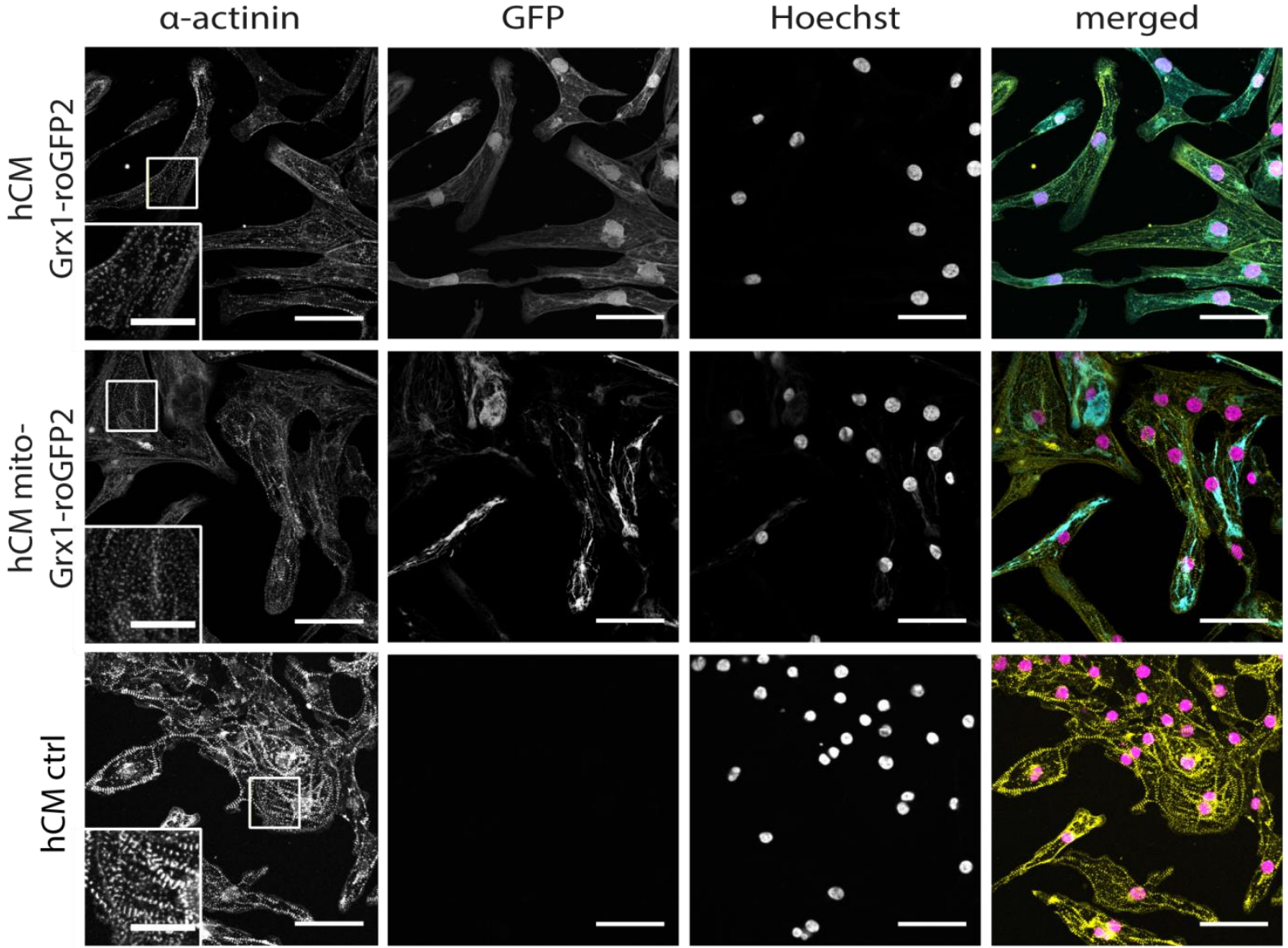

Figure 10 Transgenic HES2 derived hCM show a sarcomeric phenotype. HES2 cyto\#3 and HES2 mito2\#34 were differentiated into cardiomyocytes. A) Flow cytometry analysis of $\alpha$-actinin expression showed high differentiation efficiency and purity of cardiomyocytes. Error bars: SEM, $n=3$. B) Immunofluorescence staining of $\alpha$-actinin unmasks a disturbed sarcomere assembly in sensor line derived cardiomyocytes, Insets show magnified view of sarcomeric striations, scale bars: $20 \mu \mathrm{m}$. Figure and legend adapted from (Trautsch et al., 2019). 


\subsubsection{Stem cell derived cardiomyocytes have more oxidized mitochondria}

Despite the observed phenotype, we checked the function of expressed redox sensors. Redox imaging of cardiomyocytes derived from HES2 cyto\#3 and HES2 mito2\#34 was performed, using $100 \mathrm{\mu M} \mathrm{H}_{2} \mathrm{O}_{2}$ for oxidation and $1 \mathrm{mM}$ DTT for reduction. Cytosolic sensor Grx1-roGFP2 reacted to $100 \mu \mathrm{M} \mathrm{H}_{2} \mathrm{O}_{2}$ with an increase of fluorescence ration $\mathrm{R}_{(405 / 488)}$ to $3.3 \pm 0.01, \mathrm{n}=176$ (Figure $11 \mathrm{~A}$ ). Fluorescent ratio decreased to $0.9 \pm 0.002(n=170)$, when $1 \mathrm{mM}$ DTT was applied. Mitochondria displayed a smaller oxidation response of $2.0 \pm 0.1(n=325)$, upon treatment with $100 \mu M \mathrm{H}_{2} \mathrm{O}_{2}$, while minimum ratio after reduction with $1 \mathrm{mM}$ DTT was $0.6 \pm 0.005(n=325)$ (Figure 11 B). These findings indicate a more oxidized mitochondrial milieu in comparison to cytosol, which appears to be almost fully reduced at baseline. For further confirmation of these findings, a broader range of concentrations of $\mathrm{H}_{2} \mathrm{O}_{2}$ and DTT would need to be tested, in order to ensure maximum oxidation and reduction of the probe. As HES2 cyto\#3 and HES2 mito2\#34 derived cardiomyocytes displayed functional deficits, we decided to not investigate their redox milieu further.

A

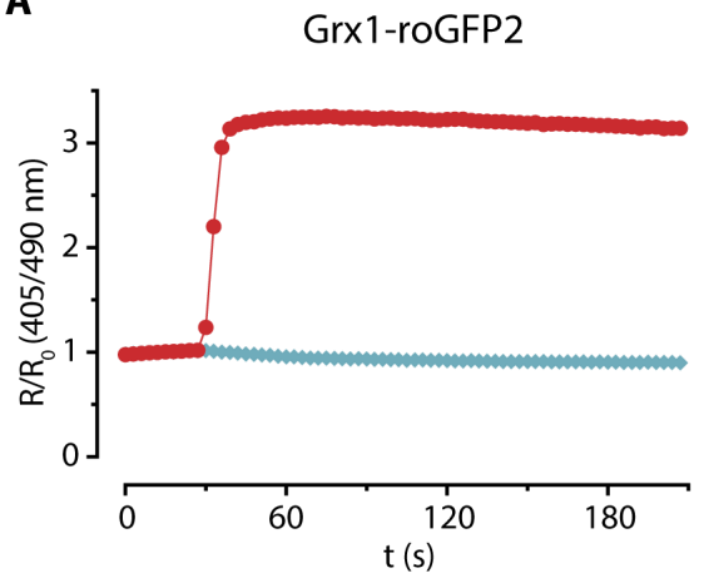

B

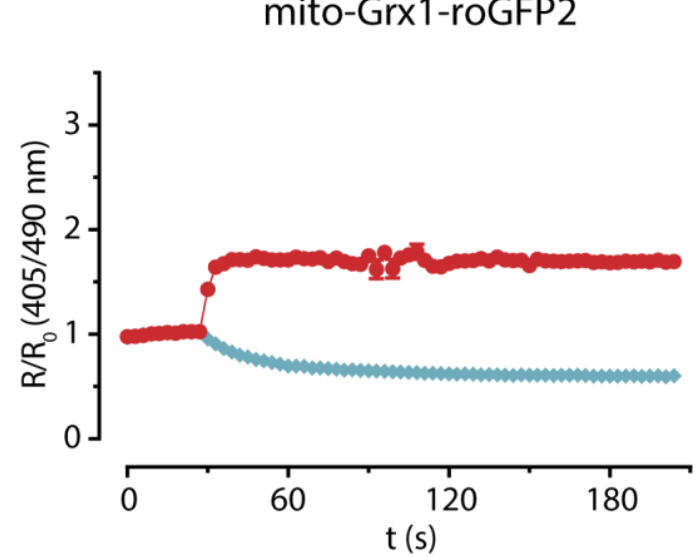

$100 \mathrm{MMH}_{2} \mathrm{O}_{2}$

$\smile \quad 1 \mathrm{mMDTT}$

Figure 11 Redox sensors are functional in stem cell derived cardiomyocytes. Fluorescence intensity upon excitation with $405 \mathrm{~nm}$ and $488 \mathrm{~nm}$ in redox sensor HES2 line derived cardiomyocytes was recorded every $3 \mathrm{~s}$ and is displayed as the change of fluorescence ratio $(R)$ over baseline $\left(R_{0}\right)$ as a function of time. $\mathrm{H}_{2} \mathrm{O}_{2}$ and DTT were added after 30 s baseline recording in the indicated concentrations. Error bars: SEM, $n=170$ - 325. Figure and legend adapted from (Trautsch et al., 2019).

\subsubsection{Redox imaging of engineered heart muscle}

It has previously been shown that 3D culture can mature cardiomyocytes and improve their function (Tiburcy et al., 2017). In an attempt to restore function of HES2 cyto\#3 and HES2 mito2\#34 derived cardiomyocytes, we generated engineered heart muscle from these cells together with primary human foreskin fibroblasts (HFF). To this end, cardiomyocytes and HFF were embedded in a collagen hydrogel and cast in ring shaped molds to allow tissue formation. EHM were then transferred to flexible stretchers, allowing further maturation by auxotonic force generation during the 28 day 
culture period (Figure $12 \mathrm{~A}$ ). In line with the observed disturbed sarcomere phenotype and the lack of contractility in monolayer culture, no contractile force was generated by redox sensor EHM during and after 28 days of maturation. However, sensor expression was retained during culture both in HES2 cyto\#3 EHM as well as in HES2 mito2\#34 EHM (Figure 12 B). In a proof-of-principle experiment, HES2mito2\#34 EHM were subjected to redox imaging. Treatment with $100 \mu \mathrm{M} \mathrm{H} \mathrm{H}_{2} \mathrm{O}_{2}$ led to a detectable increase in fluorescence intensity in $405 \mathrm{~nm}$ excitation and a concomitant reduction of fluorescent intensity in $488 \mathrm{~nm}$ excitation. Conversely, reduction with $1 \mathrm{mM}$ DTT had the opposite effect, leading to increased fluorescence intensity upon $488 \mathrm{~nm}$ excitation and reduced fluorescence intensity upon $405 \mathrm{~nm}$ excitation (Figure $12 \mathrm{C}, \mathrm{D}$ ). 
A

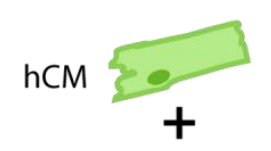

HFF

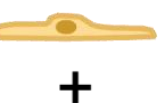

Coll
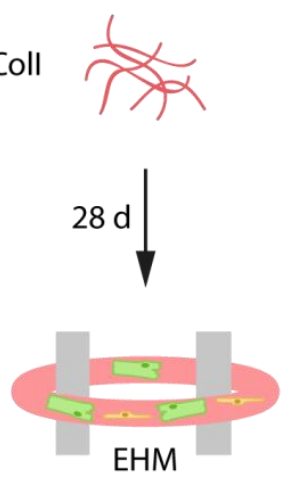

C

$\mathrm{t}=0 \mathrm{~s}$

$t=150 s$

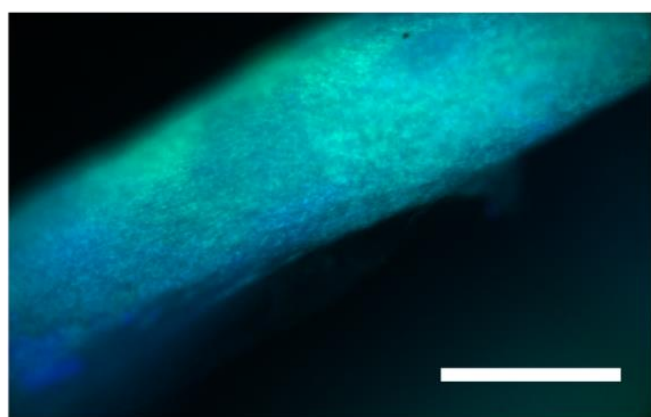

$100 \mu \mathrm{M} \mathrm{H}_{2} \mathrm{O}_{2}$
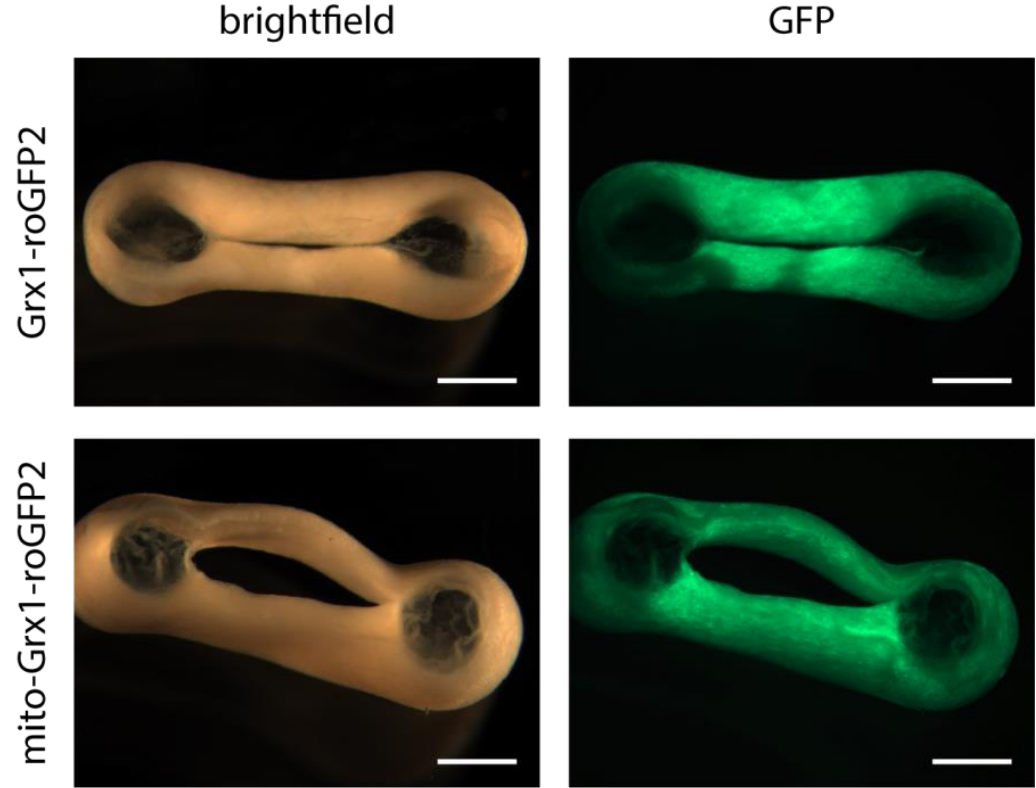

$1 \mathrm{mM}$ DTT

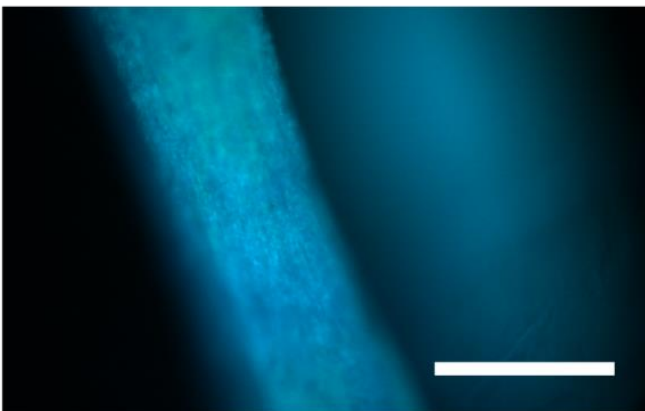

D

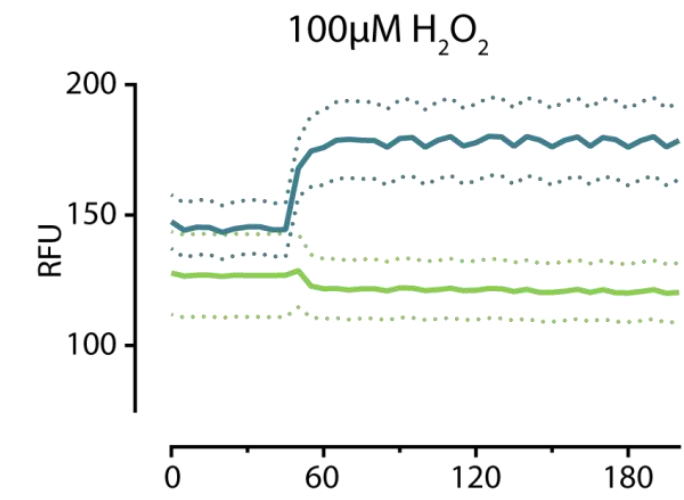

$\mathrm{t}(\mathrm{s})$
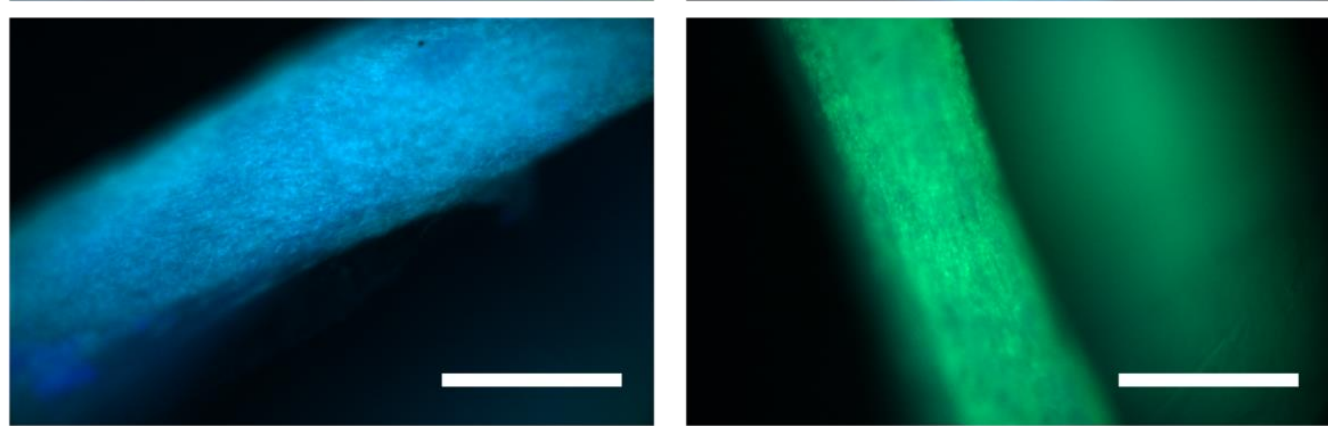

$-405 \mathrm{~nm}=488 \mathrm{~nm}$
$1 \mathrm{mM}$ DTT
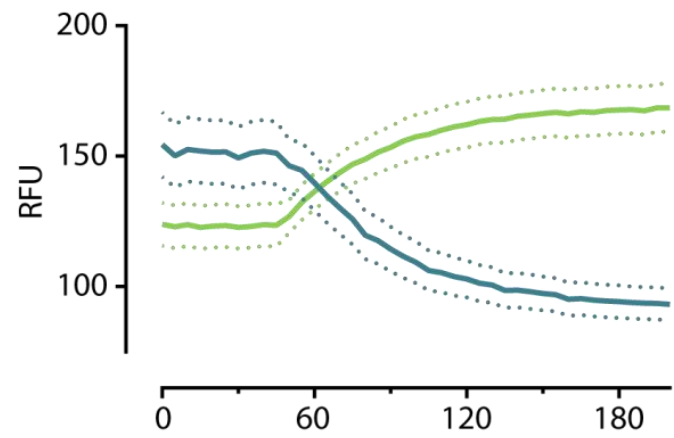

$\mathrm{t}(\mathrm{s})$ 
Figure 12 Redox signals are detectable in EHM. EHM were constructed from HES2 cyto\#3 and HES2 mito2\#34 derived cardiomyocytes. A) Schematic of EHM generation. Cardiomoyocytes (hCM) and fibroblasts (HFF) are embedded in a collagen (Coll) matrix. Ring shaped tissues are allowed to mature for 28 days on flexible stretchers. B) Brightfield and GFP images of 28 days old transgenic EHM shows retention of GFP expression during EHM maturation. Scale bars: $1 \mathrm{~mm}$. C) False colored images of EHM redox imaging of HES2 mito2\#34 EHM. Blue color represents $405 \mathrm{~nm}$ excitation, green color $488 \mathrm{~nm}$ excitation. The shift in excitation maximum upon oxidation $\left(100 \mu \mathrm{M} \mathrm{H}_{2} \mathrm{O}_{2}\right)$ and reduction (1 mM DTT) can be appreciated. Scale bars: $500 \mu \mathrm{m}$. D) Quantification of fluorescence intensities upon oxidation $\left(100 \mu \mathrm{M} \mathrm{H}_{2} \mathrm{O}_{2}\right)$ and reduction (1 mM DTT) in HES2 mito2\#34 EHM. $\mathrm{H}_{2} \mathrm{O}_{2}$ and DTT were added at 50 s. Solid lines: mean, dotted lines: SEM, $\mathrm{n}=3$ ROIs on one EHM.

Although we were not able to induce spontaneous beating by generation of EHM, we were able to demonstrate the applicability of fluorescent redox sensors in EHM. We were able to attain sensor readouts in oxidation and reduction scenarios, highlighting how redox sensors can be used to investigate redox homeostasis from undifferentiated cells to differentiated derivates and 3D engineered tissues. Further studies are required to decipher the mechanism underlying the disturbance in cardiomyocytes function.

\subsubsection{Lentivirus mediated expression of mito-Grx1-roGFP2}

As we had previously established lentivirus mediated expression of Grx1-roGFP2 in differentiated, functional cardiomyocytes (Heta, 2017), we decided to further investigate mitochondria redox potential using mito-Grx1-roGFP2 lentivirus. Lentiviral particles for the expression of Grx1-roGFP2 and mito-Grx1-roGFP2 were produced in TSA cells. Concentrated viral particles were used for the transduction of HFF and hCM. Correct localization of expressed sensors was confirmed by TMRM staining of mitochondria in live cells. In HFF, mitochondria appeared to form filamentous networks spread throughout the cells. The same pattern was detectable for mito-Grx1-roGFP2, while Grx1roGFP2 filled the cytoplasm and nucleus (Figure $13 \mathrm{~A}$ ). A high background fluorescence signal in the $405 \mathrm{~nm}$ channel was detected when analyzing nuclear staining using Hoechst, which did not colocalize with either GFP or TMRM staining. The same was later apparent in redox imaging experiments, but did not impact evaluation. As lentiviral transduction did not occur in all cells, only a subset of the imaged HFF was GFP positive.

Redox imaging of both sensors was performed in HFF. Upon treatment with $1 \mathrm{mM} \mathrm{H}_{2} \mathrm{O}_{2}$, maximum oxidized fluorescence ratio in the cytosol was $2.5 \pm 0.1, n=17$, whereas mitochondria maximum oxidized ratio was $2.3 \pm 0.1, n=28$ (Figure $13 \mathrm{C}, \mathrm{D}$ ). In the cytosol, treatment with $100 \mu \mathrm{M} \mathrm{H}_{2} \mathrm{O}_{2}$ and $10 \mu \mathrm{M} \mathrm{H}_{2} \mathrm{O}_{2}$ led to ratio increases up to $2.2 \pm 0.1, \mathrm{n}=17$, and $1.8 \pm 0.1, \mathrm{n}=21$, respectively. In contrast, mitochondria reacted to $100 \mu \mathrm{M} \mathrm{H}_{2} \mathrm{O}_{2}$ with a ratio increase close to maximal oxidation of $2.6 \pm 0.1, \mathrm{n}=29$, while $10 \mu \mathrm{M} \mathrm{H}_{2} \mathrm{O}_{2}$ only induced a slight increase of up to $1.2 \pm 0.1, \mathrm{n}=25$, indicating a threshold for full oxidation between $10 \mu \mathrm{M}$ and $100 \mu \mathrm{M} \mathrm{H}_{2} \mathrm{O}_{2}$. Conversely, application of DTT led to a decrease in fluorescence ratio (Figure $13 \mathrm{E}, \mathrm{F}$ ). Minimum reduced ratio as achieved by application of $10 \mathrm{mM}$ DTT was $0.7 \pm 0.02, n=18$, in cytosol and $0.6 \pm 0.02, n=18$, in mitochondria. Interestingly, 
application of $100 \mu \mathrm{M}$ DTT led to an initial ratio increase of Grx1-roGFP2 signal $(1.1 \pm 0.04, n=12)$, while mito-Grx1-roGFP2 ratio decreased constantly over imaging time down to $0.9 \pm 0.01, \mathrm{n}=27$. $1 \mathrm{mM}$ DTT led to an intermediate ratio decrease to $0.7 \pm 0.03, n=20$, in cytosol and $0.7 \pm 0.01$, $\mathrm{n}=27$, in mitochondria. In summary, these observations demonstrate a similar redox milieu in fibroblast mitochondria and cytosol with distinct kinetics when exposed to sub-maximal concentrations of reducing and oxidizing agents. 
A
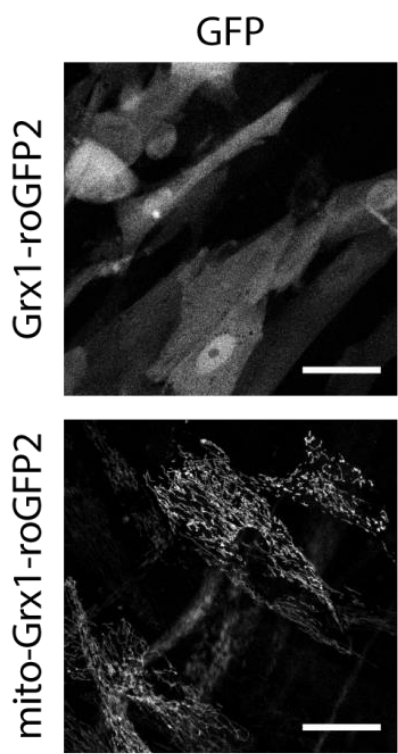

B
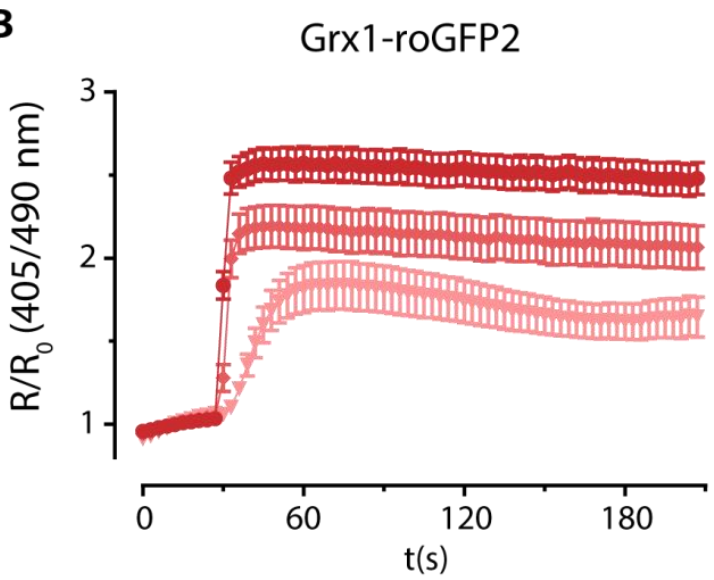

Hoechst
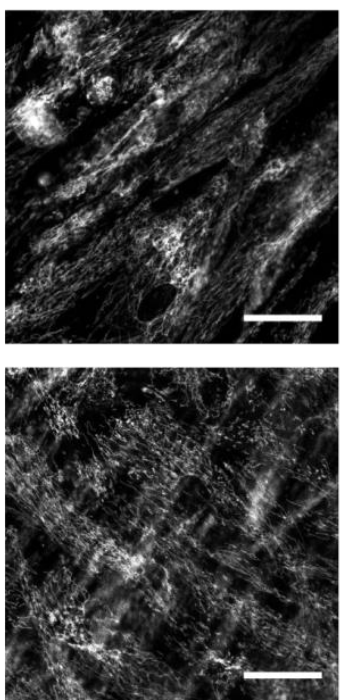
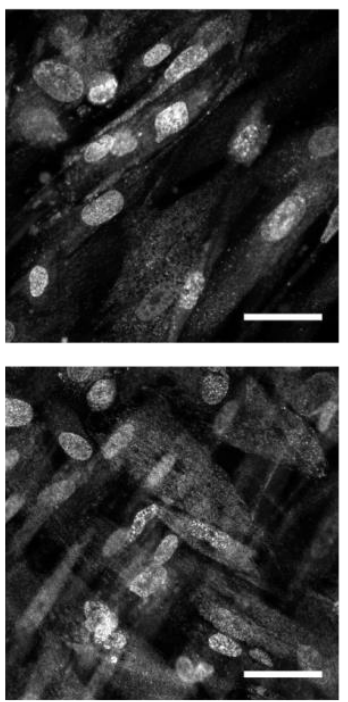

merged
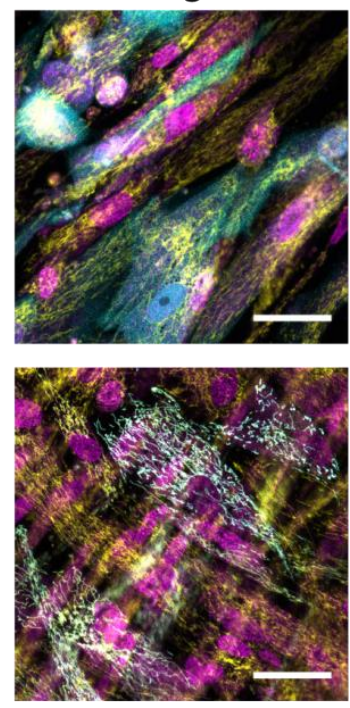

C
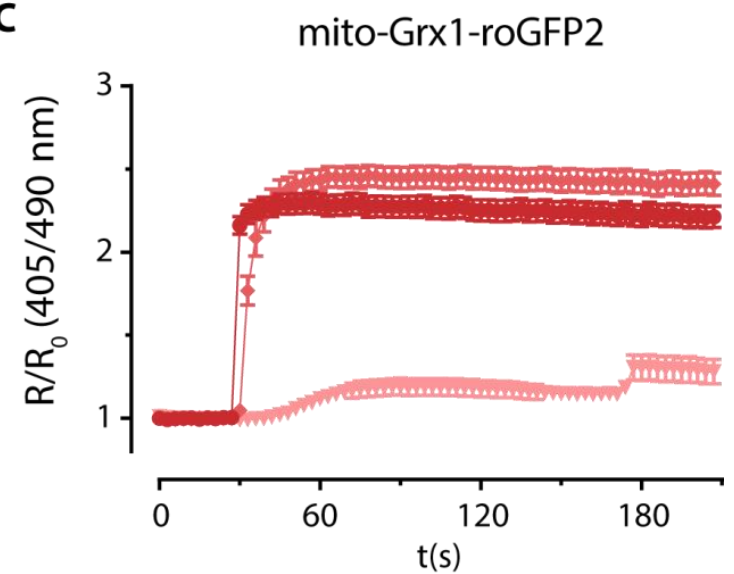

$\rightarrow 1 \mathrm{mM} \mathrm{H}_{2} \mathrm{O}_{2} \rightarrow 100 \mu \mathrm{MH}_{2} \mathrm{O}_{2} \rightarrow 10 \mu \mathrm{M} \mathrm{H}_{2} \mathrm{O}_{2}$

D

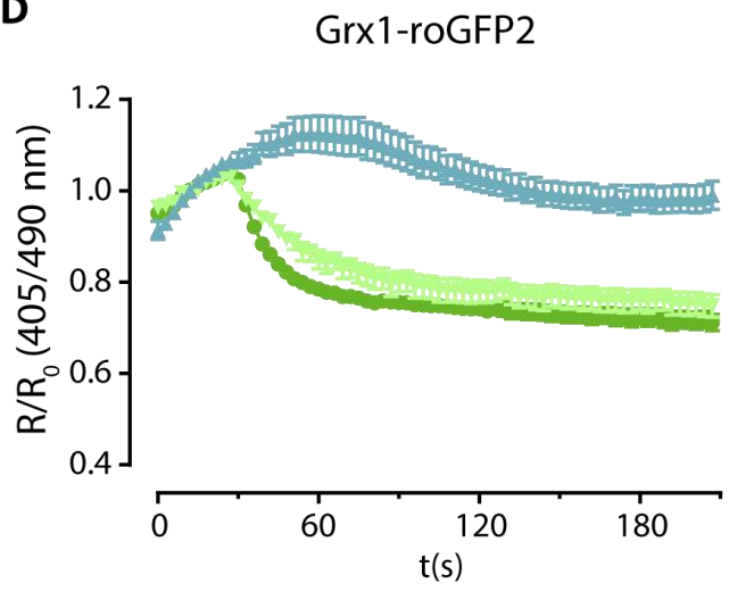

E

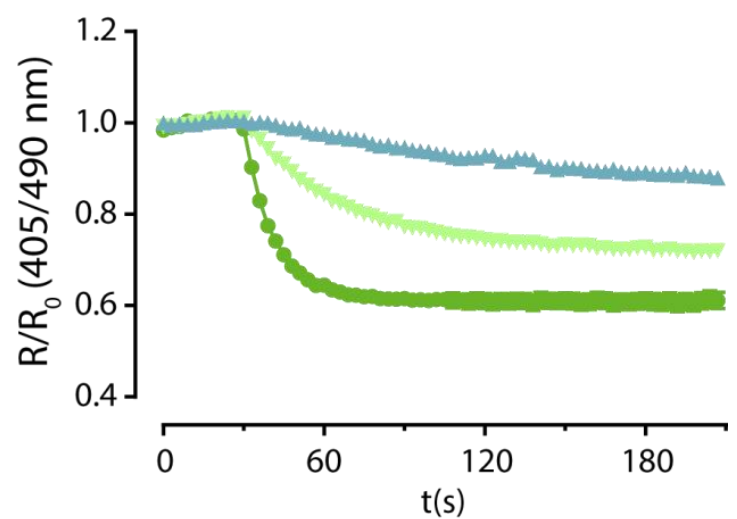

$\rightarrow 10 \mathrm{mMDT} \quad-1 \mathrm{mMDTT} \quad \longleftarrow 100 \mu \mathrm{MDT}$

Figure 13 Transduced HFF report oxidation and reduction in cytosol and mitochondria A) Live fluorescence images of roGFP2 expression, nuclei (Hoechst) and mitochondria (TMRM) in HFF transduced with lentivirus for Grx1-roGFP2 or mitoGrx1-roGFP2 showed localized expression of redox sensors. Note the high fluorescent background in the Hoechst channel $(405 \mathrm{~nm})$. Scale bar $=50 \mu \mathrm{m}$. B- E) Transduced HFF were subjected to redox imaging. Fluorescence intensity after excitation with $405 \mathrm{~nm}$ and $488 \mathrm{~nm}$ was recorded every $3 \mathrm{~s}$ and is displayed as the change of fluorescence ratio (R) over 
baseline $\left(\mathrm{R}_{0}\right)$ as a function of time. $\mathrm{H}_{2} \mathrm{O}_{2}$ or DTT were added at $30 \mathrm{~s}$ at the indicated concentrations. Error bars: SEM, $n=16-27$.

Based on our experience with Grx1-roFP2 lentiviral expression in cardiomyocytes and results obtained from mito-Grx1-roGFP2 expression in HFF, differentiated, functional cardiomyocytes were transduced with concentrated lentiviral particles for cytosolic and mitochondrial Grx1-roGFP2. Costaining of live cells with TMRM and Hoechst demonstrated mitochondria localization of mito-Grx1roGFP2 (Figure $14 \mathrm{~A}$ ). Cardiomyocytes in both conditions were however small and rounded and mitochondria looked clustered and swollen. Despite the immature morphology, beating was observed in Grx1-roGFP2 and mito-Grx1-roGFP2 transduced cells, which indicated correct cellular function. In mito-Grx1-roGFP2 expressing cardiomyocytes, fluorescence signal was also detected in the mitochondria under $405 \mathrm{~nm}$ excitation.

Subsequently, redox imaging was performed to investigate cardiomyocyte redox milieu in cytosol and mitochondria. Upon treatment with $1 \mathrm{mM} \mathrm{H}_{2} \mathrm{O}_{2}$, fluorescent ratio increased up to $3.7 \pm 0.1$, $\mathrm{n}=47$, in cytosol, while mitochondria showed less capacity for oxidation with a maximum oxidized ratio of $2.0 \pm 0.1, n=81$ (Figure $14 \mathrm{~A}, \mathrm{~B}$ ). This observation was confirmed when $10 \mathrm{mM}$ DTT was applied to induce maximal reduction. Mito-Grx1-roGFP2 fluorescent ratio decreased to $0.5 \pm 0.01$ ( $n=97$ ), while cytosolic fluorescent ratio was $0.8 \pm 0.03,(n=28$, Figure $14 C, D)$. While application of an intermediate concentration of $1 \mathrm{mM}$ DTT led to a ratio decrease in both compartments ( $0.9 \pm 0.01, n=50$, for cytosol, $0.5 \pm 0.02, n=60$, for mitochondria), $100 \mu M$ DTT led to a ratio increase of Grx1-roGFP2 up to $1.5 \pm 0.04(n=42)$. This is in contrast to previously reported results by our group which did not report an oxidative response upon treatment with low concentrations of DTT in stem cell derived cardiomyocytes. As expected, mito-Grx1-roGFP2 fluorescence ratio decreased by treatment with $100 \mu \mathrm{M}$ DTT to $0.7 \pm 0.01, \mathrm{n}=44$. Taken together, we observed a very distinct redox milieu in cytosol and mitochondria of stem cell derived cardiomyocytes. This indicates a very tight control of cellular redox milieu that might be directly linked to cellular function. 
A
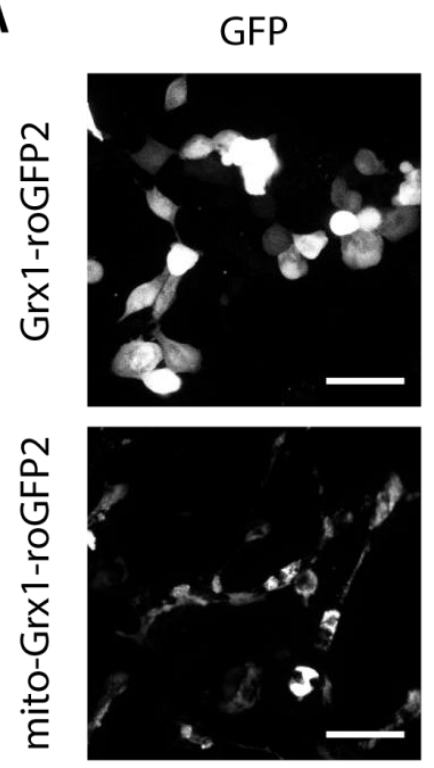

B

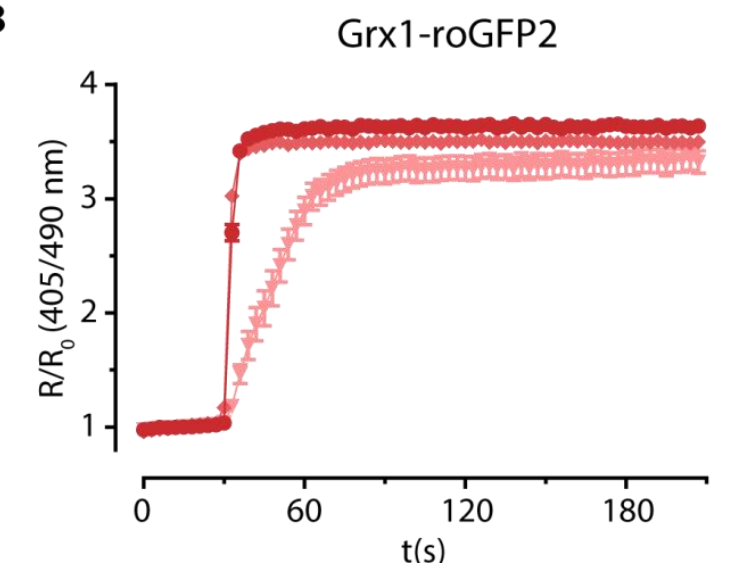

TMRM
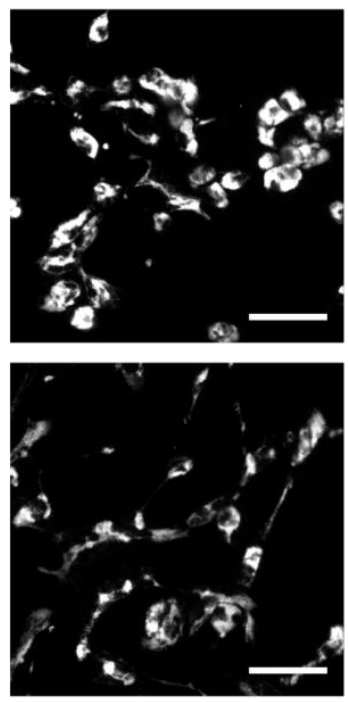

C
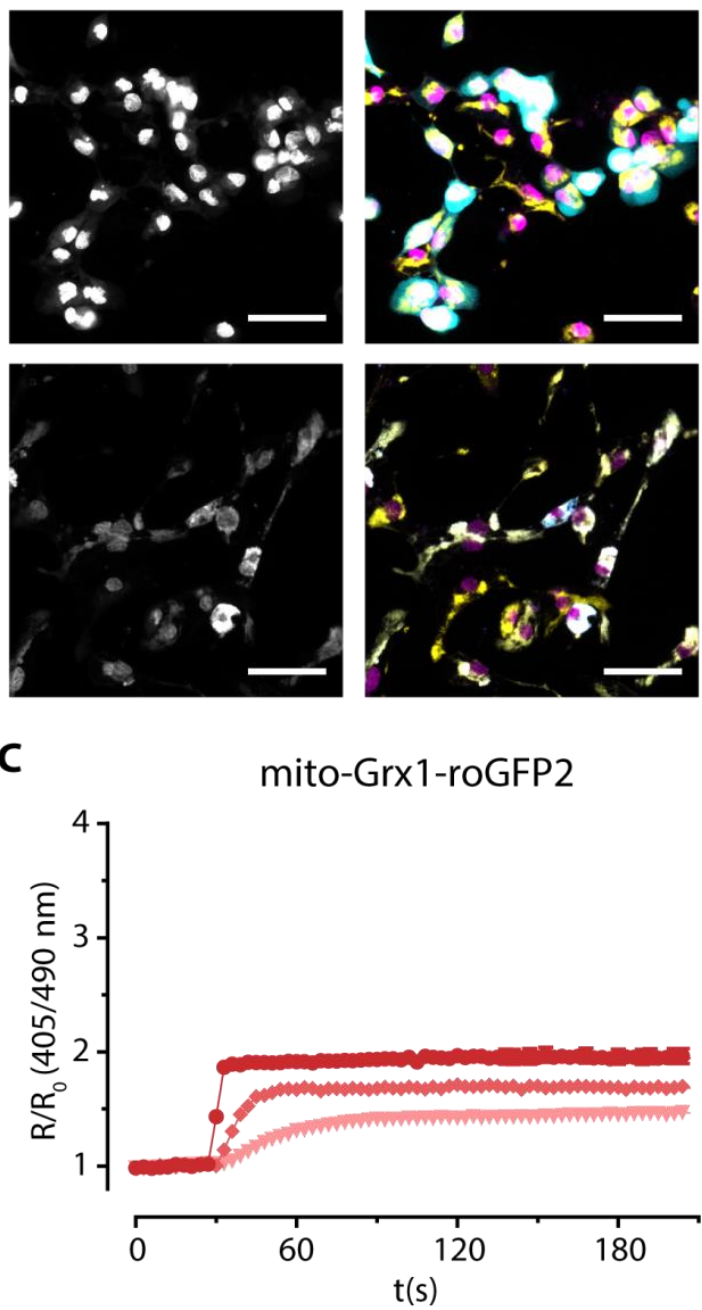

$\rightarrow 1 \mathrm{mMH}_{2} \mathrm{O}_{2} \rightarrow 100 \mu \mathrm{MH}_{2} \mathrm{O}_{2} \rightarrow 10 \mu \mathrm{MH}_{2} \mathrm{O}_{2}$

D

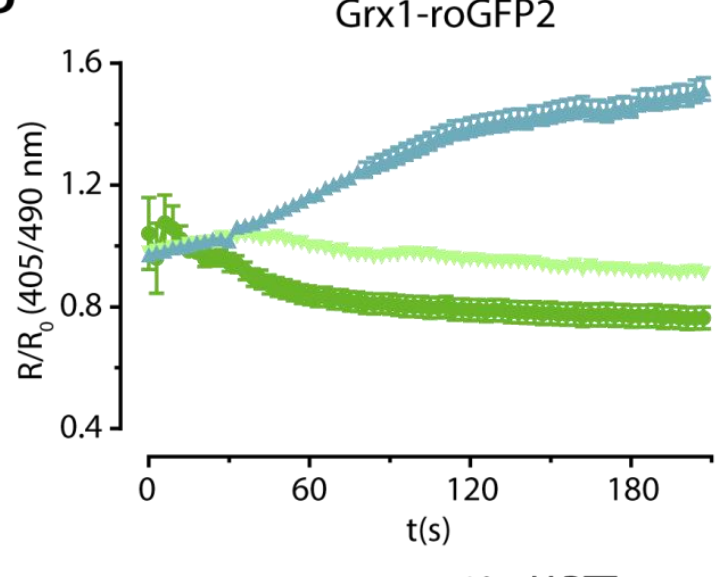

E

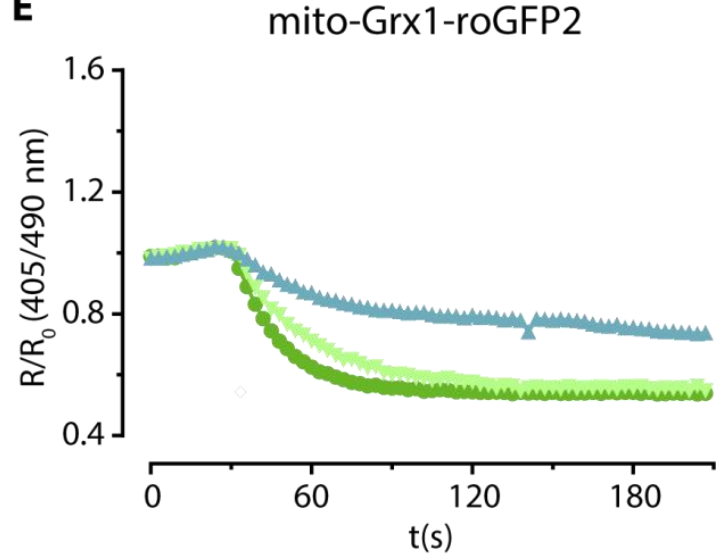

$\multimap 10 \mathrm{mMDTT}=1 \mathrm{mMDTT} \leftarrow 100 \mu \mathrm{MDTT}$

Figure 14 Transduced hCM mitochondria are oxidized compared to cytosol. A) Live fluorescence imaging of GFP, nuclei (Hoechst) and mitochondria (TMRM) confirmed the compartment specific sensor expression after lentiviral transduction. roGFP2 fluorescence in mitochondria is also detected in the $405 \mathrm{~nm}$ channel (Hoechst). Scale bar: $50 \mu \mathrm{m}$. B-E) Cardiomyocytes transduced with lentivirus encoding Grx1-roGFP2 or mito-Grx1-roGFP2 were subjected to redox imaging. Fluorescence intensity after excitation with $405 \mathrm{~nm}$ and $488 \mathrm{~nm}$ was recorded every $3 \mathrm{~s}$ and is displayed as the change of 
fluorescence ratio $(R)$ over baseline $\left(R_{0}\right)$ as a function of time. $\mathrm{H}_{2} \mathrm{O}_{2}$ or DTT were added at $30 \mathrm{~s}$ at the indicated concentrations. Error bars: SEM, $\mathrm{n}=20-97$.

\subsection{Sensing and producing $\mathrm{H}_{2} \mathrm{O}_{2}$ in cell-cell interaction models}

While GSH sensors allow for monitoring of GSH/GSSG redox homeostasis in cells and tissues, they are limited as they only report changes in a cellular buffer system. Minor, more dynamic changes are likely happening at the level of individual ROS, such as $\mathrm{H}_{2} \mathrm{O}_{2}$. One genetically encoded sensor to monitor $\mathrm{H}_{2} \mathrm{O}_{2}$ in cells is HyPer. Apart from monitoring changes in cellular $\mathrm{H}_{2} \mathrm{O}_{2}$ levels, a novel fusion protein of HyPer and a D-amino acid oxidase, HyPerDAO offers the intriguing possibility to also manipulate intracellular ROS levels. It stands to reason that production of $\mathrm{H}_{2} \mathrm{O}_{2}$ inside the cell will follow different kinetics and exert different ROS stress on the cell than an application of $\mathrm{H}_{2} \mathrm{O}_{2}$ to the culture medium. In the second part of this thesis, we established expression of HyPer and HyPerDAO in TSA and HES cells and demonstrated function of sensor-producer complexes.

\subsubsection{HyPerDAO produces $\mathrm{H}_{2} \mathrm{O}_{2}$ in TSA cells}

We generated donor vectors for the genetic integration of HyPer and HyPerDAO. TSA cells were transfected with linearized donor plasmid and plasmids encoding components of a TALEN system targeting the AAVS1 safe harbor locus. Successful transfection gave rise to TSA HyPer and TSA HyperDAO cells with uniform expression of fluorescent redox sensors as detectable by GFP flow cytometry (Figure 15).

TSA HyPer and TSA HyPerDAO were seeded on optical bottom 96-well plates and plate reader based redox assays performed. In both lines, HyPer reacted to oxidation by increasing concentrations of $\mathrm{H}_{2} \mathrm{O}_{2}$ with an increase in fluorescence ratio $\mathrm{R}_{(405 / 488)}$. Maximum oxidation was achieved by $100 \mu \mathrm{M}$ $\mathrm{H}_{2} \mathrm{O}_{2}$ and led to a ratio increase to $3.3 \pm 0.2, \mathrm{n}=12$, in HyPer (Figure $16 \mathrm{~A}$ ) and $3.4 \pm 0.2, \mathrm{n}=12$, in HyPerDAO cells (Figure 16 B). $1 \mu \mathrm{M} \mathrm{H}_{2} \mathrm{O}_{2}$ did not induce oxidation of the sensor, while $10 \mu \mathrm{M} \mathrm{H}_{2} \mathrm{O}_{2}$ led to a moderate increase of fluorescence ratio. Upon treatment with DTT, no change in fluorescent ratio was observed $(0.94 \pm 0.02, n=12$ for HyPer and $0.93 \pm 0.02, n=12$ for HyPerDAO; Figure $16 C$, D). It has been postulated that intracellular HyPer is reduced by components of the GSH system. As previously observed with cytosolic Grx1-roGFP2 expression in TSA cells, the cytosolic GSH/GSSG pool is already highly reduced at baseline. The same might be true for HyPer in this case. 
A

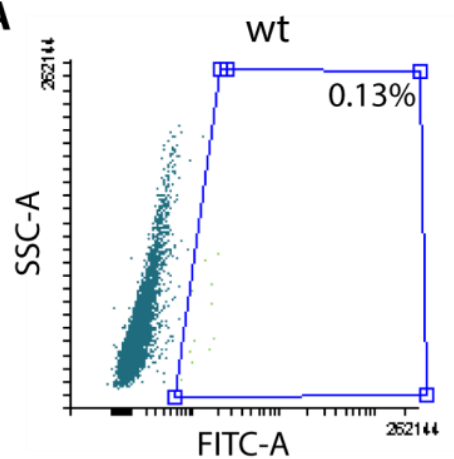

FITC-A

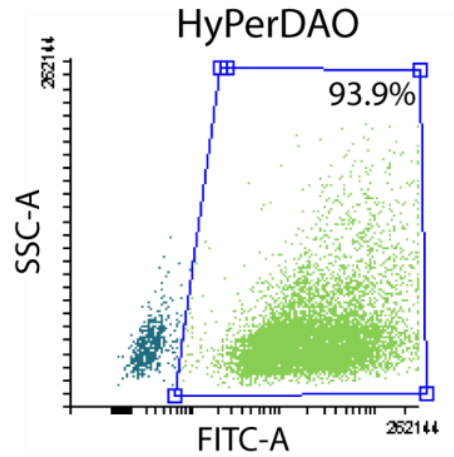

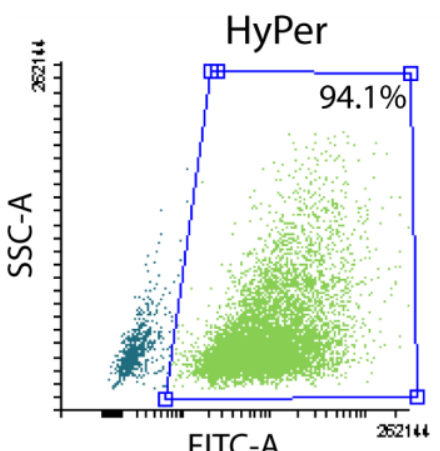

FITC-A

B

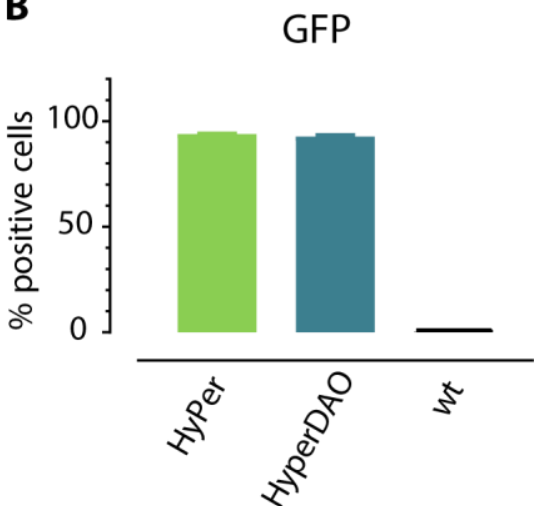

Figure 15 TSA cells express HyPer and HyPerDAO. A) Example dot plots of live cell flow cytometry shows uniform transgene expression. B) Transgene expression is stable over passages and uniform in HyPer and HyPerDAO TSA cells. Live flow cytometry analysis, error bars: SEM, $\mathrm{n}=3$.

When D-amino acids such as D-alanine are present, DAO will oxidize these amino acids to their corresponding pyruvic acid. In this reaction, $\mathrm{H}_{2} \mathrm{O}_{2}$ is generated as a byproduct (Figure $16 \mathrm{E}$ ). When adding $8 \mathrm{mM}$ D-alanine to HyPerDAO TSA cells, the produced $\mathrm{H}_{2} \mathrm{O}_{2}$ leads to an increase in fluorescent ratio $\mathrm{R}_{(405 / 488)}$ to $2.3 \pm 0.2, \mathrm{n}=12$ (Figure $16 \mathrm{~F}$ ). This increase is not visible in cells expressing only HyPer $\left(\mathrm{R}_{(405 / 488)}=1.0 \pm 0.02, \mathrm{n}=12\right)$. HyPerDAO thus allows for the targeted production of $\mathrm{H}_{2} \mathrm{O}_{2}$ restricted to cells harboring this specific transgene. 

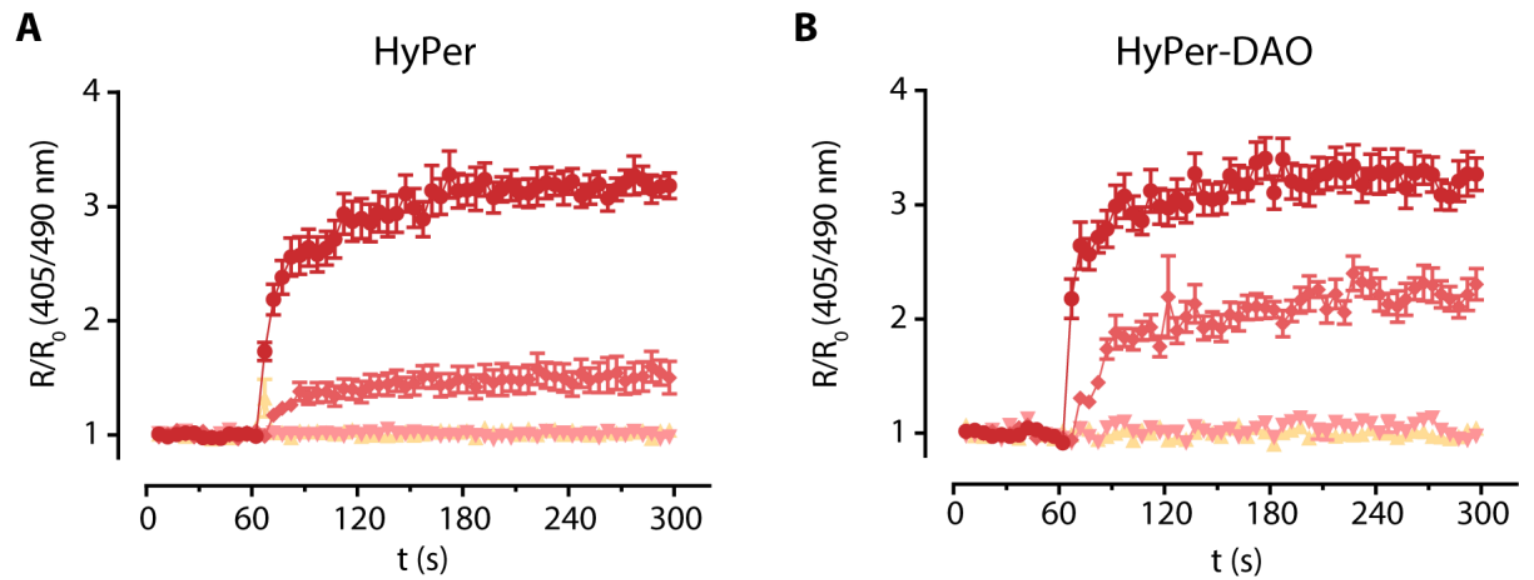

- $100 \mu \mathrm{MH}_{2} \mathrm{O}_{2}$

$\rightarrow 10 \mu \mathrm{MH}_{2} \mathrm{O}_{2}$

$-1 \mu \mathrm{MH}_{2} \mathrm{O}_{2}$

ctrl

C

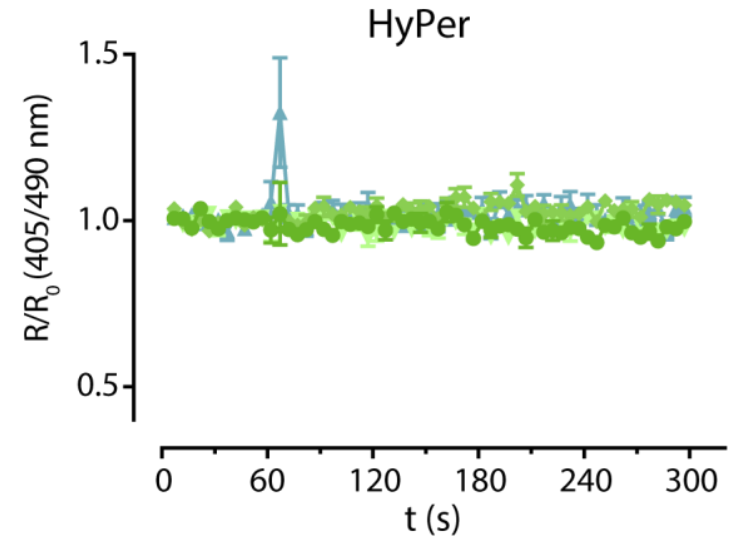

D

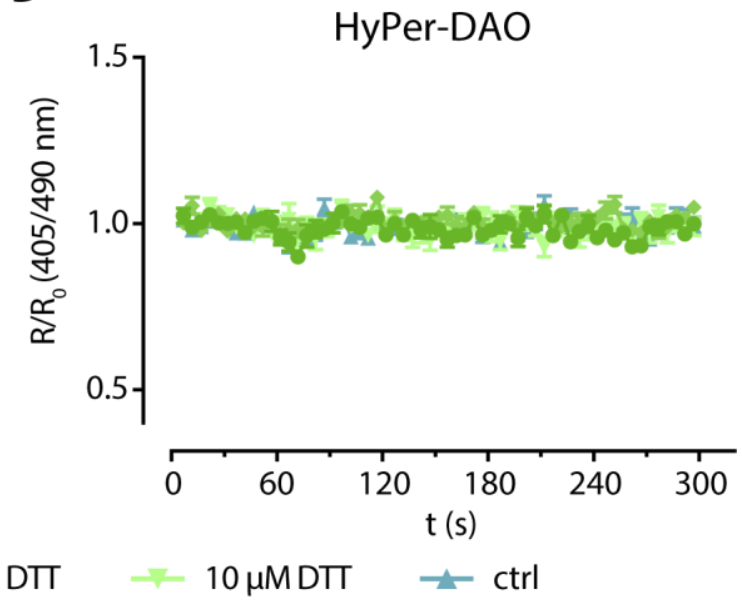

E

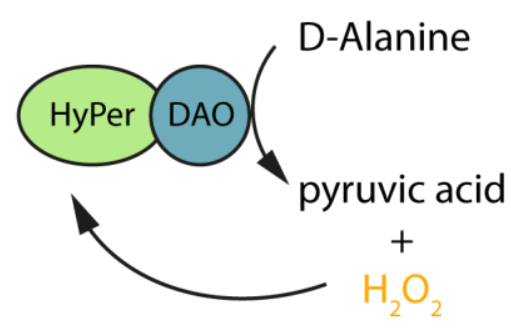

F

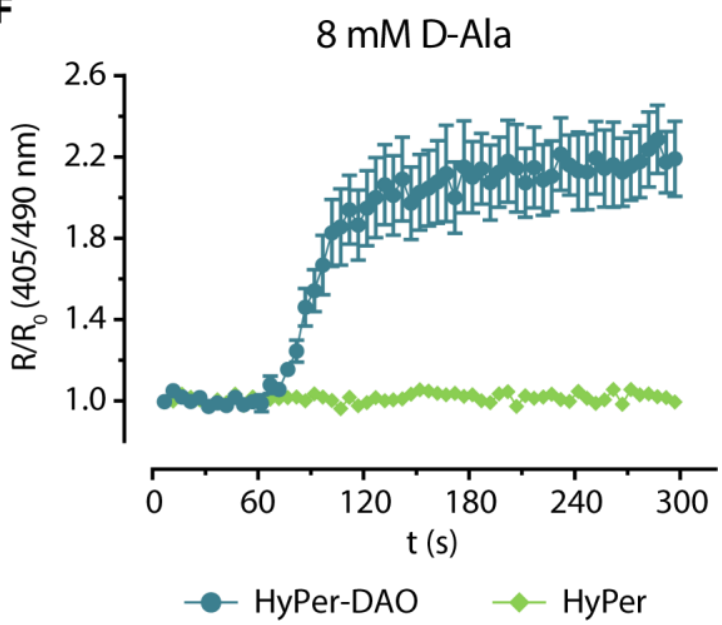

Figure 16 HyPer and HyPerDAO is functional in TSA cells. TSA cells expressing either HyPer (A, C) or HyPerDAO (B, D) were treated with indicated concentrations of $\mathrm{H}_{2} \mathrm{O}_{2}$ and DTT. Fluorescence intensity upon excitation with $405 \mathrm{~nm}$ and $488 \mathrm{~nm}$ was recorded every $5 \mathrm{~s}$ and is displayed as the change of fluorescence ratio $(R)$ over baseline $\left(R_{0}\right)$ as a function of time. $\mathrm{H}_{2} \mathrm{O}_{2}$ and DTT were added after 60 s. Error bars: SEM, $\mathrm{n}=11$ - 12. E) HyPerDAO acts as a sensor-producer hybrid for $\mathrm{H}_{2} \mathrm{O}_{2}$. The oxidation of D-alanine by DAO gives rise to $\mathrm{H}_{2} \mathrm{O}_{2}$ which is in turn detected by HyPer. F) Cells expressing HyPer or HyperDAO were treated with $8 \mathrm{mM}$ D-alanine at $60 \mathrm{~s}$ and fluorescence intensitiy recorded for excitation wavelenghts $405 \mathrm{~nm}$ and $488 \mathrm{~nm}$. Only when DAO is present, an increase in cellular $\mathrm{H}_{2} \mathrm{O}_{2}$ concentration is detected. Error bars: SEM, $\mathrm{n}=12$. 


\subsubsection{HES2 integrate and express HyPer expression sequences}

As HyPer and HyPerDAO offered intriguing possibilities to analyze and manipulate the redox homeostasis in stem cell derived cardiomoycytes, we set out to generate stem cell lines expressing HyPer or HyPerDAO.

HES2 were electroporated with linearized donors vectors for HyPer and HyperDAO as well as two plasmids encoding a TALEN system targeting the AAVS1 locus. Single cells clones were selected based on fluorescence and genotyping for genomic integration performed (Figure 17 C, D). Based on the results, one HES2 line expressing HyPer (HES2 HyPer\#5) and one line expressing HyperDAO (HES2 HyPerDAO\#5) were chosen for further investigation. Pluripotency was confirmed by flow cytometry analysis of Oct4, Nanog and Tra1-60 expression. Both transgenic lines showed similarly high expression of pluripotency markers as non-transgenic control cells (Figure $17 \mathrm{~A}$ ). The correct expression of sensor protein was further confirmed by Western blot analysis using a GFP antibody. The larger HyPerDAO fusion protein (estimated molecular weight $93 \mathrm{kDa}$ ) was clearly separated from HyPer, which is around $53 \mathrm{kDa}$ in size (Figure $17 \mathrm{~B}$ ).

A

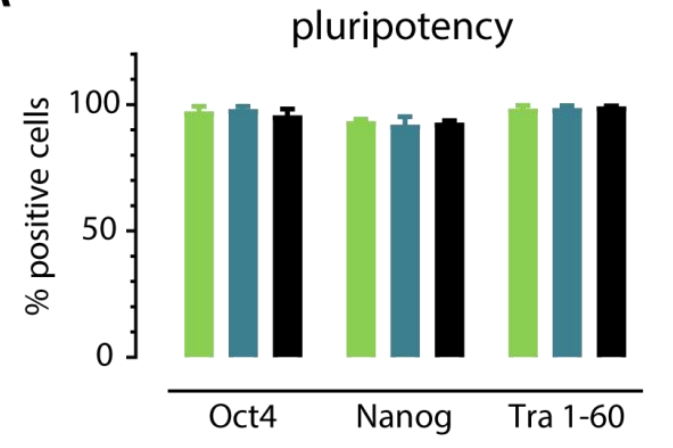

HES2 HyPer\#5 HES2 HyPer-DAO\#5

B

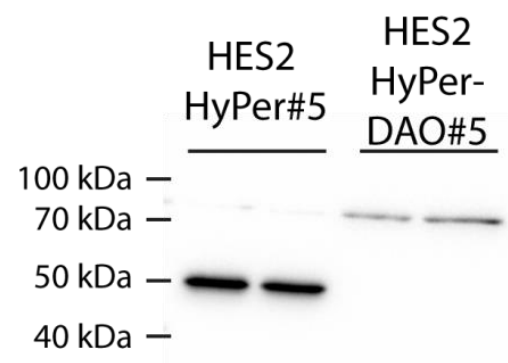

C

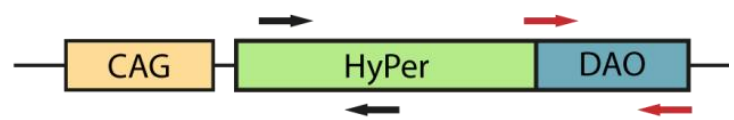

D

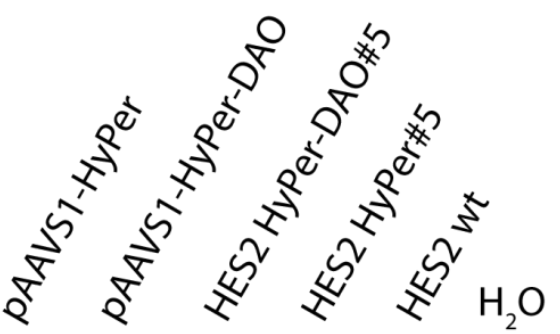

Figure 17 HyPer expression in HES2 does not influence pluripotency. A) Pluripotency analysis of HES lines expressing HyPer (HES2 HyPer\#5) or HyPerDAO (HES2 HyPerDAO\#5) shows expression of pluripotency markers Oct4, Nanog and Tra 160 after transgene integration. B) Western blot demonstrates expression of HyPer and HyPerDAO transgenes in analyzed lines. Due to the fusion with DAO, HyPerDAO is approximately $93 \mathrm{kDa}$, whereas HyPer is $53 \mathrm{kDa}$ in size. C) Schematic of duplex PCR for genotyping of transgenic lines. Two primer pairs were used in a single PCR reaction giving rise to one band for HyPer (black arrows, 171 bp) and one band for DAO (red arrows, 296 bp). D) Representative agarose gel electrophoresis for genotyping of HES2 lines. pAAVS1 donor vectors are used as positive control. 
Similarly to the Grx1-roGFP2 transgenic lines, we were not able to verify integration in the AAVS1 locus for HES2 HyPer\#5 and HES2 HyPerDAO\#5. This led us to assume that also in this case, transgenes most likely integrated randomly. We performed test differentiations of HyPer and HyPerDAO lines towards cardiomyocytes, but in line with roGFP2 lines, no spontaneous beating was observed. We therefore decided to discontinue experiments with these lines and focus on a more efficient CRISPR/Cas9 approach for genomic integration. 


\section{Discussion}

Redox signaling plays a major role in cardiac homeostasis and disease. The coordinated production of ROS in a defined spatial and temporal manner by NOXs can exert manifold effects on cardiomyocyte function. Contractility, metabolism and even gene transcription are regulated by redox modifications of key signaling proteins. ROS production by mitochondria can also influence these pathways and is an integral part of metabolic regulatory networks. Measurements of cellular ROS are however limited by the use of traditional dye based detection systems, which do not allow for repeated and long term studies. With the development of fluorescent redox sensors, tools for the dynamic, in-situ analysis of ROS and the cellular ROS scavenging systems are available.

In the present study, we investigated the use of genetically encoded redox sensors in human pluripotent stem cells, human dermal fibroblasts, differentiated cardiomyocytes and engineered heart muscle. HES2 lines with genetic integration of GSH redox sensors Grx1-roGFP2 and mito-Grx1roGFP2 as well as $\mathrm{H}_{2} \mathrm{O}_{2}$ sensor HyPer and sensor-producer hybrid HyPerDAO were generated. As cardiomyocytes differentiated from these lines displayed deficits in sarcomere assembly, in addition lentiviral transduction of stem cell derived cardiomyocytes and dermal fibroblasts for compartment specific expression of Grx1-roGFP2 was performed. Redox imaging revealed differences in GSH/GSSG balance between stem cells, cardiomyocytes and fibroblasts, highlighting the importance of cell type specific redox imaging.

The main findings of this study are:

- Genetic integration of redox sensors does not impair pluripotent stem cell marker expression, but impairs sarcomere assembly of differentiated cardiomyocytes.

- Analysis of Grx1-roGFP2 and mito-Grx1-roGFP2 by redox imaging allows for the determination of compartment specific GSH/GSSG redox balance in human embryonic stem cells, human dermal fibroblasts, stem cell derived cardiomyocytes and engineered heart muscle.

- In undifferentiated human embryonic stem cells as well as human stem cell derived cardiomyocytes, the mitochondria GSH/GSSG pool is more oxidized than the cytosol.

- In contrast to cardiomyocytes, fibroblast GSH/GSSG balance appears similar between cytosol and mitochondria.

- $\mathrm{H}_{2} \mathrm{O}_{2}$ sensor-producer construct HyPerDAO allows for targeted manipulation of cellular redox balance. 


\subsection{Genomic integration in embryonic stem cells}

In order to establish a pipeline for the investigation of cellular redox milieus in human stem cell derived cardiomyocytes and EHMs, genetic integration of redox sensors in human embryonic stem cells was performed. Although a TALEN system targeting the AAVS1 locus on human chromosome 19 was used for the integration of redox sensors, transgenes integrated randomly. In principle it is not expected that this leads to an impact on cardiomyogenesis, however, we observed an impaired beating function upon differentiation of transgenic HES lines. This phenotype cannot be explained completely by random transgene integration, as we analyzed integration sites and found no cardiac association of the genes or regions disrupted. Furthermore, traditional transgene models such as the Grx1-roGFP2 mouse model (Swain et al., 2016) often rely on random integration of target genes. Usually, tissue specific promotors are chosen for the expression of the integrated transgenes. However, in our model, the ubiquitous promotor CAG leads to a strong expression at all stages of differentiation. For future applications a more reliable system for transgene integration would be beneficial as the AAVS1 locus has been described as a safe harbor integration site, whose disruption is not associated with a disease phenotype (Oceguera-Yanez et al., 2016). A more efficient CRISPR/Cas9 system targeting the same locus has successfully been used by other members of our working group in the meantime and showed reliable locus integration (Schoger, unpublished). It remains unclear however, whether expression from single copy integration would be sufficient to measure redox sensor signals.

A previous study made use of lentiviral transduction for the expression of redox sensors in cardiomyocytes (Heta, 2017; Trautsch et al., 2019). This is a fast and reliable protocol for stable invitro transgene expression and was also used in this study to corroborate findings from transgenic HES lines. The need for constant virus production and cell transduction makes this approach tedious when considering constant cell requirements for large scale experiments. In addition, low transduction efficiency, varying viral titers and differing expression levels due to multiple infections of the same cell can all influence reproducibility and reliability of data. Furthermore, when considering tissue engineering approaches, the need for large numbers of cells with homogenous sensor expression becomes even more apparent. Nevertheless, we utilized lentiviral particles to express Grx1-roGFP2 and mito-Grx1-roGFP2 in stem cell derived cardiomyocytes and fibroblasts as a fast approach to test the toxicity of redox sensor expression in already differentiated cardiomyocytes. 


\subsection{Cardiac differentiation is deficient in transgenic HES lines}

One major concern with fluorescent redox sensors is that by overexpressing a protein containing reactive cysteines, cellular redox homeostasis can be changed. The redox sensor itself might act as a buffering system for ROS and thus influence cellular signaling. ROS signaling has been implicated in stem cell maintenance and function (Bigarella et al., 2014). In general, fast cycling stem cells rely on glycolysis and pentose phosphate pathway to fulfill their metabolic demands. As oxidative phosphorylation is suppressed, the amount of generated ROS by metabolic processes is very low and the cellular milieu reduced, as determined by analysis of total protein oxidative modification (Prigione 2010). In fact, it has been postulated that an activation of oxidative phosphorylation in pluripotent stem cells can lead to a loss of stemness by increasing ROS (Bigarella et al., 2014). It has been shown that mitochondrial maturation with increasing oxidative phosphorylation occurs very early in differentiation, together with an increase in ROS production (Cho et al., 2006; Mandal et al., 2011). Mitochondrial maturation as observed by network formation occurs even before pluripotency marker expression decreases (Mandal et al., 2011). Conversly, blocking oxidative phosphorylation by ectopic expression of uncoupling protein 2 decreases ROS formation during differentiation and reduces overall differentiation capacity (Zhang et al., 2011). We did not observe a decline in stem cell marker expression in our transgenic lines during culture time, on the contrary, we observed high and robust pluripotency marker expression. Therefore, constitutive redox sensor overexpression might lead to a buffering of ROS signals important for differentiation initiation, which causes a stabilization of pluripotency and blocks differentiation.

Despite various studies, the exact effects of ROS on cardiac differentiation remain unclear. A number of studies support the notion that an increase in cellular ROS will enhance cardiac differentiation. In undirected, spontaneous differentiation approaches utilizing embryoid bodies, application of $\mathrm{H}_{2} \mathrm{O}_{2}$ or ROS generator menadione was shown to enhance the emergence of beating areas, whereas ROS scavengers such as $\mathrm{N}$-acetylcysteine or vitamin $\mathrm{E}$ derivate Trolox appeared to inhibit cardiac differentiation (Sauer et al., 2000). $\mathrm{H}_{2} \mathrm{O}_{2}$ was also able to induce cardiac differentiation and maturation in isolated murine embryonic cardiomyocytes in a NOX dependent mechanism (Buggisch et al., 2007). A number of studies attributed the cardiogenic effect observed with different compounds to cellular ROS increase. It was shown that Cardiothrophin-1 increased ROS in mouse embryonic stem cell derived embryoid bodies which led to the activation of NF-kappaB signaling cascades (Sauer et al., 2004). General antioxidant treatment with vitamin E or specific NOX inhibition abrogated this effect (Sauer et al., 2004). A similar mechanism was reported for icariin induced cardiomyogenesis, which could also be inhibited by administration of NOX inhibitors or Trolox (Ding et al., 2008). Icariin was further shown to induce NOX4 expression and p38 mitogen activated kinase 
driven nuclear translocation of cardiac transcription factor myocyte enhancer factor $2 \mathrm{C}$ (Ding et al., 2008). Another study observed an increase in cellular ROS by treatment of cells with a peroxisome proliferator-activated receptor $\alpha$ agonist that also induced cardiac differentiation. Also in this model, the cardioinstructive effect could be inhibited by treatment with NOX inhibitors or general antioxidants (Sharifpanah et al., 2008). While all these studies argue for an important role of NOX derived ROS in cardiac differentiation, others have shown that antioxidant treatments can also increase cardiomyocyte yield. An early screening study found that ascorbic acid will enhance cardiac differentiation while $\mathrm{N}$-acetylcysteine or vitamin $\mathrm{E}$ are without effect (Takahashi et al., 2003). Others have shown that the embryotoxic compound valproic acid will increase ROS in an embryoid body model while reducing cardiac differentiation efficiency ( $\mathrm{Na}$ et al., 2003). Vitamin E was able to rescue this effect by reducing the observed increase in ROS (Na et al., 2003). In a model of peroxisome proliferator-activated receptor $\gamma$ coactivator $1 \alpha$ knock down, which led to a decrease in cellular mitochondrial content, ROS level was decreased while cardiomyocytes showed an improved $\mathrm{Ca}^{2+}$ transient amplitude that indicated a more mature phenotype (Birket et al., 2013). Treatment with Nacetylcysteine or incubation at lower oxygen concentrations induced a similar effect. Taking these results into account, it becomes evident that the role of ROS in cardiac differentiation is not easy to pinpoint. Results will depend on the model studied and the protocol used for differentiation. As most studies use undefined, spontaneous differentiation protocols that utilize high serum media, it is unclear what other compounds might influence cardiac differentiation efficiency. Furthermore, these protocols yield only very small percentages of cardiomyocytes, sometimes below $10 \%$ of all cells. Considering the cell type specificity of redox milieus that we report in this study, it is questionable how informative redox measurements with typically used dye based systems of these mixed differentiations can be in respect to the redox milieu of the small subset of cells committed to a cardiac fate. In order to fully understand how ROS act in cardiac differentiation, studies of single ROS species in defined, highly efficient differentiation protocols are needed. A recent study underlines the need for more targeted analysis of ROS in cardiac differentiation (Tu et al., 2018). Thiol-containing antioxidants $\beta$-mercaptoethanol and monothiolglycerol were shown to reduce cardiac differentiation efficiency in spontaneous embryoid body differentiation, while vitamin $E$ derivate Trolox enhanced cardiomyocyte numbers. All compounds were able to reduce cellular ROS, but only Trolox treatment led to a decrease in mitochondrial superoxide. Interestingly, $\beta$ mercaptoethanol and monothiolglycerol even enhanced mitochondria superoxide production (Tu et al., 2018). This indicates that general levels of ROS are not a reliable read-out when analyzing the redox effects of any given treatment. It is rather necessary to investigate the individual ROS in defined cell populations or even compartments within cells in order to gain insight in the redox 
regulation of cardiac differentiation. With redox sensor transgenic stem cells we possess the ability to analyze redox milieus in differentiating cells under such conditions in a dynamic, compartment specific manner. The observation of disturbed sarcomerogenesis in transgenic cardiomyocytes is in line with a ROS buffering effect by the sensor and the role of ROS in cardiomyocyte differentiation and maturation. Thus it appears that constitutive expression of redox sensors has an impact on cell fate.

Should the contractile phenotype of transgenic lines observed in this study be caused by the constitutive expression of redox sensors, it will be very early stages of differentiation that are influenced, as several cardiomyocyte specific models of redox sensor expression have been reported. In line with this we did not observe impaired cardiomyocyte function after lentiviral transduction with Grx1-roGFP2 or mito-Grx1-roGFP2 of differentiated cells. Similarly, in animal models using adenoviral overexpression no disturbances were observed (Dey et al., 2018; Steinhorn et al., 2017). In addition, a mouse model with expression of Grx1-roGFP2 and mito-Grx1-roGFP2 under the control of the cardiac $\alpha \mathrm{MHC}$ promotor did not show any cardiac phenotype after transgene integration (Swain et al., 2016). Cells committed to a cardiomyocyte fate thus seem to be insensitive to redox sensor overexpression. Further experiments would be needed to clarify at which step differentiation is impaired in transgenic sensor cardiomyocytes. In addition, technical reasons for the failure of cardiac differentiation cannot be excluded. We selected only a single clone of each line to undergo further characterization and differentiation, which may have resulted in a selection bias for cells with unknown genetic or epigenetic mutations hindering cardiomyocyte function. It seems however unlikely that this occurred in all four analyzed lines (HES2 cyto\#3, mito3\#34, HyPer\#5 and HyPerDAO\#5), but screenings of other clones might lead to clarity in this case. For mito-Grx1-roGFP2 we were able to obtain beating cells in two out of twelve differentiations, indicating that also further optimization of the differentiation protocol, e.g. by ROS modulation, might be needed, as differentiation of these cells is in principle possible.

\subsection{The GSH/GSSG redox balance in cardiomyocyte mitochondria}

We investigated the GSH/GSSG redox milieu in cytosol and mitochondria in stem cells and stem cell

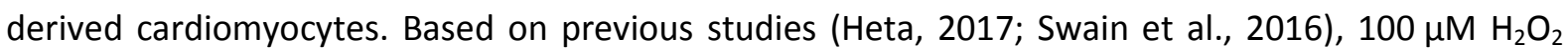
and $1 \mathrm{mM}$ DTT were used for maximum oxidation and reduction in preliminary TSA experiments. As cytosolic Grx1-roGFP2 appeared insensitive to DTT treatment, we increased the maximum DTT concentration used to $10 \mathrm{mM}$ for subsequent HES and cardiomyocyte experiments. Using such high amounts allowed us to confidently determine the minimum reduced ratio in undifferentiated stem cells and cardiomyocytes as necessary for redox potential calculation. Concomittantly, we increased 
$\mathrm{H}_{2} \mathrm{O}_{2}$ maximum concentration to $300 \mu \mathrm{M}$ for HES2 measurements and to $1 \mathrm{mM}$ in cardiomyocyte measurements. Interestingly, cardiomyocytes appeared to be already maximally oxidized when treated with $100 \mu \mathrm{M} \mathrm{H}_{2} \mathrm{O}_{2}$, in line with previous studies (Heta, 2017). Undifferentiated stem cells however reported an increased oxidation when treated with $300 \mu \mathrm{M} \mathrm{H}_{2} \mathrm{O}_{2}$ compared to $100 \mu \mathrm{M}$ $\mathrm{H}_{2} \mathrm{O}_{2}$. This indicates a need for careful titration of compound concentration for each cell type analyzed in order to achieve maximum oxidation and reduction. In order to further confirm that maximal oxidation is reached, treatment with the thiol oxidant diamide can be performed. By directly oxidizing cysteine thiol residues, diamide does not act via the GSH/GSSH relay for oxidation of Grx1-roGFP2. Therefore, influences of the GSH/GSSG system on sensor readout are excluded. In a previous study, we did not observe major differences between the maximum oxidized ratio that could be achieved by $\mathrm{H}_{2} \mathrm{O}_{2}$ treatment in comparison to diamide (Heta, 2017). Although the concentrations in this study are in line with published results, they represent supraphysiological concentrations. The intracellular concentration of $\mathrm{H}_{2} \mathrm{O}_{2}$ is estimated to be in the range of $10 \mathrm{~nm}$ to $100 \mathrm{~nm}$ in physiological signaling and increase up to $1 \mu \mathrm{M}$ in acute oxidative stress (Sies, 2017). Measurements of extracellular $\mathrm{H}_{2} \mathrm{O}_{2}$ concentration have shown that a 100 -fold higher oxidant concentration can be found in the extracellular space (Sies, 2017). This high oxidant gradient might explain why such high concentrations of extracellular applied oxidants are needed to achieve robust oxidation of intracellular redox sensor probes.

One main finding of this study was that stem cell as well as cardiomyocyte mitochondria where highly oxidized in comparison to cytosol. Interestingly, adult mouse cardiomyocytes were shown to maintain a more reduced mitochondria redox milieu in comparison to cytosol (Swain et al., 2016), while the developing zebrafish heart had previously been described to have more oxidized mitochondria (Panieri et al., 2017). Since mitochondrial ROS are tightly linked to metabolic homeostasis, mitochondrial redox milieu might play a pivotal role in cardiomyocyte metabolic maturation. Stem cell derived cardiomyocytes have been described to have a fetal metabolic phenotype with a strong reliance on glycolysis, while it is known for the adult heart to rely on fatty acid oxidation and oxidative phosphorylation to cover the high energy demand of working myocardium. Modulation of the MPTP and thus mitochondria $\Delta \psi$ by administration of cyclosporine A was demonstrated to enhance cardiomyocyte differentiation (Cho et al., 2014). The rationale behind these findings argues that an increase in $\Delta \psi$ will lead to mitochondria maturation and increased oxidative phosphorylation, which favors cardiomyogenesis. A study using engineered tissues found that supplementation of medium with fatty acids and reduction of glucose content can indeed increase cardiac maturation in a 3D model, which is accompanied by an increase in mitochondrial biogenesis and oxidative phosphorylation (Mills et al., 2017). This metabolic 
maturation occurred together with an arrest in cell proliferation induced by DNA damage response pathways. These findings recapitulate the in-vivo adaptations that occur after birth, when the mammalian heart switches from glycolytic to oxidative energy metabolism in conjunction with a sharp decrease in cardiac proliferation capacity (Girard et al., 1992; Mills et al., 2017). These studies offer insight in the intriguing link between mitochondria function and cellular fate, which is mediated by metabolic adaptations and potentially ROS. Redox sensor expressing cells might prove to be valuable tools in the investigation of metabolic and mitochondria regulation of cellular processes such as maturation, proliferation and regeneration. As a first step towards a deeper understanding of redox homeostasis in stem cells and cardiomyocytes it would be of great benefit to analyze stem cell derived cardiomyocytes in models of enhanced maturation such as the EHM. Within this thesis, proof-of-concept for redox imaging in EHM was demonstrated. It would be anticipated that culture of stem cell derived cardiomyocytes in EHM, which is known to enhance cardiac maturation, would also impact metabolic homeostasis and cellular and mitochondria redox milieu. Concomitant analysis of metabolic phenotype together with redox imaging at different stages of tissue maturation would be highly informative as to how maturation, metabolism and ROS are interlinked in human cardiomoycytes.

When comparing the results of the present study, i.e., the more oxidized mitochondria milieu in stem cell derived cardiomyocytes, to those made in studies using transgenic mice, i.e., a more reduced mitochondria milieu, species differences should not be disregarded. The mouse heart is characterized by a much faster heartbeat than the human with around $800 \mathrm{bpm}$. Such a fast heartbeat will undoubtedly lead to a very high energy demand in mouse cardiomyocytes, possibly exceeding the demand experienced by a human cardiomyocyte. Therefore, mouse cardiac mitochondria might have additional adaptations that allow for an enhanced energy production without an increase in ROS production, one of which could be the maintenance of a more reduced GSH/GSSG pool. To elucidate whether species differences underlie the observed difference in mitochondrial redox milieu, studies of fetal and neonatal mouse cardiomyocytes would be needed. By comparison of these immature cardiomyocytes to adult, mature mouse cardiomyocytes the influence of cardiac maturation on mitochondria GSH/GSSG redox homeostasis could be further investigated. If cardiac maturation is accompanied by a decrease in mitochondria redox potential, metabolic and mitochondria maturation present as key driver mechanisms of cardiac redox homeostasis. A recapitulation of these results in human stem-cell based models of cardiac maturation would be anticipated. 


\subsection{Cardiac redox homeostasis and fibroblasts}

In the heart, fibroblast activation upon injury mediates the necessary scarring for mechanical stability, but excess activation of fibroblasts leads to excessive pathological fibrosis. Pathological fibrosis is characterized by the remodeling and deposition of extracellular matrix proteins and fibroblast proliferation. It leads to an increased stiffness of heart muscle, which impairs contraction and relaxation. The increased mechanical stress causes cardiomyocytes to adapt and acts as an amplifying mechanism of cardiac dysfunction. Investigations of the determinants of fibroblasts activation and senescence in the heart have tried to find potential therapeutic targets in order to limit the fibrotic response to improve long term cardiac function. In cardiac tissue engineering, it has previously been described that stromal cells are required for the formation of functional engineered myocardium. Stromal cells provide the necessary structural extracellular matrix milieu, which is required by cardiomyocytes for functional maturation (Christalla et al., 2012; Naito et al., 2006). Fibroblasts mediate tissue consolidation already within the first $30 \mathrm{~min}$ of tissue formation (Schlick et al., 2018). Fibroblast origin as well as age have an impact on engineered cardiac tissue development and function (Li et al., 2017; Schlick, 2019). Engineered heart muscle models have also elucidated how fibroblast dysfunctions can lead to cardiac disease, for example upon loss of collagen processing protein heat shock protein 47, leading to failure of EHM formation (Sur, 2016). Considering the importance of fibroblast biology for cardiac homeostasis, we extended our study to include analysis of human dermal fibroblast redox properties by lentiviral transduction with Grx1roGFP2 and mito-Grx1-roGFP2. While redox regulation of cardiomyocyte homeostasis has been widely studied, extensive analysis of fibroblast redox properties are missing. It has been shown that transforming growth factor $\beta-1$ (TGF $\beta-1)$ stimulation increases collagen production through a mechanism dependent on cellular ROS production by NOX4. When NOX4 is inhibited or knocked down by short hairpin RNA, the effect of TGFB-1 on collagen production is reduced (Hotta et al., 2018). In addition, fibroblast activation and increased collagen secretion by Ang 2 stimulation has been shown to be dependent on NOX derived ROS (Shen et al., 2006). The regulation of fibroblast to myofibroblast transition is therefore very likely also redox mediated. As the dermal fibroblasts utilized in this study are highly proliferative, they would represent a more myofibroblast-like phenotype. When analyzing the cellular redox milieu, in contrast to cardiomyocytes or stem cells, dermal fibroblasts did not show a difference in redox homeostasis between cytosol and mitochondria. This difference underlines the importance of cell type specific studies of cellular redox signaling, as it hints towards a differential regulation of GSH/GSSG homeostasis in cardiomyocytes and fibroblasts. Future studies of fibroblast redox homeostasis might utilize engineered tissue approaches, as these resemble the in-vivo environment of fibroblasts more closely than traditional 
2D cultures on stiff matrices. Unpublished data from our lab suggest a reversal of culture induced fibroblast activation in 3D engineered connective tissues (Santos, unpublished). Furthermore, EHM offer the unique possibility to analyze the interplay between different cell types in cardiac development and function. By using either fibroblasts or cardiomyocytes expressing redox sensors to construct EHM, cell type specific redox responses to different physiological and pathophysiological stimuli could be elucidated without neglecting the cellular interactions that are also found in-vivo.

\subsection{Cell type targeted manipulation of ROS production}

Grx1-roGFP2 based sensors detect changes in the cellular main ROS buffering GSH/GSSG system and are therefore limited in their ability to display small signaling events that are characterized by fast and local production and degradation of ROS. In order to extend the scope of the thesis, the applicability of $\mathrm{H}_{2} \mathrm{O}_{2}$ sensor HyPer was tested in-vitro. In TSA cells, HyPer reacted to extracellular stimulation with concentrations above $10 \mu \mathrm{M} \mathrm{H}_{2} \mathrm{O}_{2}$ with an increase in fluorescence ratio. Studies with purified HyPer protein determined the sensitivity threshold for detection to be $25 \mathrm{nM} \mathrm{H}_{2} \mathrm{O}_{2}$, which is several orders of magnitude smaller than the values observed in this study (Belousov et al., 2006). Effects of cellular $\mathrm{H}_{2} \mathrm{O}_{2}$ clearance and membrane permeability might however reduce the actual concentration of $\mathrm{H}_{2} \mathrm{O}_{2}$ achieved inside the cell. As observed with Grx1-roGFP2 expression in TSA cells, these cells are highly reduced at baseline and might therefore possess a high buffering capacity. As HyPer is most likely reduced by cellular GSH (Zheng et al., 1998), a highly reduced GSH pool will also lead to a reduced HyPer pool, that might be insensitive to small amounts of $\mathrm{H}_{2} \mathrm{O}_{2}$.

Besides the ability to observe and quantify cellular $\mathrm{H}_{2} \mathrm{O}_{2}$ production, a sensor-producer hybrid was developed to manipulate intracellular ROS levels. The D-amino acid oxidase domain of HyPerDAO oxidizes D-amino acids under the concomitant production of $\mathrm{H}_{2} \mathrm{O}_{2}$. Initial studies determined the amount of produced $\mathrm{H}_{2} \mathrm{O}_{2}$ to be in the range of up to 50 - $100 \mathrm{nM}$ (Matlashov et al., 2014). It stands to reason that this approach will lead to different cellular signaling events than simple bolus stimulation by extracellular addition of $\mathrm{H}_{2} \mathrm{O}_{2}$ to the culture medium, as the aforementioned effects of $\mathrm{H}_{2} \mathrm{O}_{2}$ neutralization by medium components will not come into play. Furthermore, by constant supply of D-amino acids, a constant production of $\mathrm{H}_{2} \mathrm{O}_{2}$ can be achieved. This in in contrast to the short half-life of externally added $\mathrm{H}_{2} \mathrm{O}_{2}$ that will quickly degrade in aqueous solutions. By using HyPerDAO transgenic cells for engineered tissue construction, $\mathrm{H}_{2} \mathrm{O}_{2}$ production in only a subset of the used cells can be achieved. That way, cell type specific tissue responses can be analyzed.

Within this study transgenic HES lines expressing HyPer and HyPerDAO were generated. These lines were similarly dysfunctional when differentiated to cardiomyocytes as observed for the Grx1-roGFP2 models. We therefore decided to not continue with experiments in these lines, but to generate 
transgenic iPSC lines by a CRISPR integration approach that is still ongoing work. Previous experience in our lab has shown that this approach leads to improved targeting of the AAVS1 locus with high efficiency (Schoger, unpublished).

\subsection{Applicability and use of fluorescent redox sensors in redox research}

In this thesis we provide proof-of-concept for the genetic integration and expression of different fluorescent redox sensor systems in human embryonic stem cells and their differentiated derivatives. While fluorescent protein redox sensors offer many advances over dye based approaches, one of them being the ability to dynamically report redox milieu changes, they are certainly still limited in their applicability. While the expression of exogenous sequences is becoming more and more straightforward in different models, some cell types will remain difficult to transfect. The generation of transgenic models is not possible for all research questions and also viral vector mediated expression is limited. Therefore, dye based systems for ROS detection will remain a valuable tool in redox research, as they offer fast read-out and seemingly easy application. It is however crucial for all ROS detection methods that data analysis is carried out carefully bearing possible confounding factors in mind.

A common feature of the discussed methods is that they report a mere observation. Determination of the $\mathrm{H}_{2} \mathrm{O}_{2}$ production rate or GSH/GSSG status of a cell or compartment is just one data point among many that are necessary to elucidate the effect of redox signaling on cellular processes. Determining changes in cellular redox homeostasis upon certain treatments or interventions is certainly a valid strategy, but more mechanistic approaches are needed to explain the observed alterations. In this respect techniques such as redox proteomics will serve in identifying possible effector or target proteins. The rise of easy genome editing methodologies like CRISPR/Cas9 mediated mutation or knock out will facilitate the confirmation of targets by generation of "redoxdead" mutant protein sequences lacking redox active cysteines.

In respect to redox signaling and its role in the heart, this study can only serve as a primer, highlighting the importance of cell type and compartment specific analysis of individual ROS species. Especially in respect to the interplay of cardiomyocyte metabolism, maturation and redox homeostasis, we now have the necessary tools available to investigate these processes in a human system. The application of redox sensors in EHM will further enhance our understanding of cardiac redox regulation. 


\section{Summary \& Outlook}

ROS play an important role in the regulation of cellular fate and function. In cardiomyocytes, ROS are involved in processes such as differentiation, excitation-contraction coupling, hypertrophy and metabolism. In order to understand how redox signals regulate all these processes, suitable methods for the detection of ROS are necessary. Genetically encoded fluorescent protein redox sensors enable dynamic measurements of specific ROS and can easily be targeted to cellular compartments. Here, we made use of GSH sensor Grx1-roGFP2 targeted to cytosol and mitochondria, and $\mathrm{H}_{2} \mathrm{O}_{2}$ sensor HyPer in TSA, HES2, stem cell derived cardiomyocytes and dermal fibroblasts. While genetic integration in HES2 enabled the analysis of stem cell GSH/GSSG dynamics, cardiomyocytes differentiated from these lines were functionally deficient. An analysis of more clonal lines and generation of a more targeted integration by CRISPR/Cas9 might clarify reasons for this phenotype.

Toxicity of redox sensor expression for cardiomyocyte function could be excluded based on experiments performed with lentiviral transduced cardiomyocytes which retained beating. One major finding from analysis of these cells was the more oxidized mitochondrial milieu in comparison to cytosol that was observed in stem cells and cardiomyocytes. As it is known for adult mouse cardiomyocytes to retain a more reduced mitochondrial milieu (Swain et al., 2016), further studies might focus on the role of metabolic maturation and its interplay with redox biology in cardiomyocytes. Furthermore, Grx1-roGFP2 and mito-Grx1-roGFP2 cardiomyocytes will be valuable tools for the analysis of physiological and pathophysiological redox reactions towards neurohumoral, electrical and pharmacological stimulation. Also the application of ischemia-reperfusion models in cells and EHM as a well understood redox mediated damage mechanism will further increase our understanding of cardiomyocyte redox biology.

In contrast to cardiomyocytes, dermal fibroblasts did not report differences in cytosolic vs mitochondria GSH/GSSG balance, indicating a very different cellular regulation of ROS levels. Once again, these findings indicate the need for cell type specific analysis of ROS. Especially in the context of engineered tissues such as EHM, the cell type specific GSH/GSSG balance is of high interest.

Besides analyzing the $\mathrm{H}_{2} \mathrm{O}_{2}$ levels in TSA cells with HyPer, we also generated cells with expression of sensor-producer hybrid HyPerDAO, which allows for the targeted production of $\mathrm{H}_{2} \mathrm{O}_{2}$ inside the cell. This offers interesting possibilities of ROS manipulation in a cell and tissue context. Future work will focus on the generation of stable HyPer and HyPerDAO expression in iPSCs and elucidate cell type specific effects and pathologies in an EHM model. 
In conclusion, the models established and described in this study will allow for a multitude of future studies elucidating the role of ROS in cardiac physiology and disease. In combination with tissue engineering, they enable us to investigate cell type specific redox responses in a human system in a physiological setting. 


\section{Bibliography}

Abilez, O. J., Tzatzalos, E., Yang, H., Zhao, M.-T., Jung, G., Zöllner, A. M., Tiburcy, M., Riegler, J., Matsa, E., Shukla, P., Zhuge, Y., Chour, T., Chen, V. C., Burridge, P. W., Karakikes, I., Kuhl, E., Bernstein, D., Couture, L. A., Gold, J. D., Zimmermann, W. H., \& Wu, J. C. (2018). Passive Stretch Induces Structural and Functional Maturation of Engineered Heart Muscle as Predicted by Computational Modeling. Stem Cells (Dayton, Ohio), 36(2), 265-277.

Acín-Pérez, R., Fernández-Silva, P., Peleato, M. L., Pérez-Martos, A., \& Enriquez, J. A. (2008). Respiratory Active Mitochondrial Supercomplexes. Molecular Cell, 32(4), 529-539.

Basili, S., Tanzilli, G., Mangieri, E., Raparelli, V., Di Santo, S., Pignatelli, P., \& Violi, F. (2010). Intravenous ascorbic acid infusion improves myocardial perfusion grade during elective percutaneous coronary intervention: relationship with oxidative stress markers. JACC. Cardiovascular Interventions, 3(2), 221-229.

Belousov, V. V., Fradkov, A. F., Lukyanov, K. A., Staroverov, D. B., Shakhbazov, K. S., Terskikh, A. V., \& Lukyanov, S. (2006). Genetically encoded fluorescent indicator for intracellular hydrogen peroxide. Nature Methods, 3(4), 281-286.

Bendall, J. K., Cave, A. C., Heymes, C., Gall, N., \& Shah, A. M. (2002). Pivotal role of a gp91(phox)containing NADPH oxidase in angiotensin II-induced cardiac hypertrophy in mice. Circulation, 105(3), 293-296.

Bennett, C. N., Ross, S. E., Longo, K. A., Bajnok, L., Hemati, N., Johnson, K. W., Harrison, S. D., \& MacDougald, O. A. (2002). Regulation of Wnt Signaling during Adipogenesis. Journal of Biological Chemistry, 277(34), 30998-31004.

Bigarella, C. L., Liang, R., \& Ghaffari, S. (2014). Stem cells and the impact of ROS signaling. Development (Cambridge, England), 141(22), 4206-4218.

Bilan, D. S., Pase, L., Joosen, L., Gorokhovatsky, A. Y., Ermakova, Y. G., Gadella, T. W. J., Grabher, C., Schultz, C., Lukyanov, S., \& Belousov, V. V. (2013). HyPer-3: a genetically encoded H(2)O(2) probe with improved performance for ratiometric and fluorescence lifetime imaging. ACS Chemical Biology, 8(3), 535-542.

Birket, M. J., Casini, S., Kosmidis, G., Elliott, D. A., Gerencser, A. A., Baartscheer, A., Schumacher, C., Mastroberardino, P. G., Elefanty, A. G., Stanley, E. G., \& Mummery, C. L. (2013). PGC-1 $\alpha$ and reactive oxygen species regulate human embryonic stem cell-derived cardiomyocyte function. Stem Cell Reports, 1(6), 560-574.

Biteau, B., Labarre, J., \& Toledano, M. B. (2003). ATP-dependent reduction of cysteine-sulphinic acid by S. cerevisiae sulphiredoxin. Nature, 425(6961), 980-984. 
Boch, J., Scholze, H., Schornack, S., Landgraf, A., Hahn, S., Kay, S., Lahaye, T., Nickstadt, A., \& Bonas, U. (2009). Breaking the code of DNA binding specificity of TAL-type III effectors. Science (New York, N.Y.), 326(5959), 1509-1512.

Brandes, R. P., Weissmann, N., \& Schröder, K. (2014). Nox family NADPH oxidases: Molecular mechanisms of activation. Free Radical Biology and Medicine, 76, 208-226.

Brejc, K., Sixma, T. K., Kitts, P. A., Kain, S. R., Tsien, R. Y., Ormö, M., \& Remington, S. J. (1997). Structural basis for dual excitation and photoisomerization of the Aequorea victoria green fluorescent protein. Proceedings of the National Academy of Sciences of the United States of America, 94(6), 2306-2311.

Buggisch, M., Ateghang, B., Ruhe, C., Strobel, C., Lange, S., Wartenberg, M., \& Sauer, H. (2007). Stimulation of ES-cell-derived cardiomyogenesis and neonatal cardiac cell proliferation by reactive oxygen species and NADPH oxidase. Journal of Cell Science, 120(5), 885-894.

Burgoyne, J. R., \& Eaton, P. (2009). Transnitrosylating nitric oxide species directly activate type I protein kinase $A$, providing a novel adenylate cyclase-independent cross-talk to betaadrenergic-like signaling. The Journal of Biological Chemistry, 284(43), 29260-29268.

Burridge, P. W., Matsa, E., Shukla, P., Lin, Z. C., Churko, J. M., Ebert, A. D., Lan, F., Diecke, S., Huber, B., Mordwinkin, N. M., Plews, J. R., Abilez, O. J., Cui, B., Gold, J. D., \& Wu, J. C. (2014). Chemically defined generation of human cardiomyocytes. Nature Methods, 11(8), 855-860. Burridge, P. W., Thompson, S., Millrod, M. A., Weinberg, S., Yuan, X., Peters, A., Mahairaki, V., Koliatsos, V. E., Tung, L., \& Zambidis, E. T. (2011). A universal system for highly efficient cardiac differentiation of human induced pluripotent stem cells that eliminates interline variability. PloS One, 6(4), e18293.

Chattoraj, M., King, B. A., Bublitz, G. U., \& Boxer, S. G. (1996). Ultra-fast excited state dynamics in green fluorescent protein: multiple states and proton transfer. Proceedings of the National Academy of Sciences, 93(16), 8362-8367.

Chen, B., Dodge, M. E., Tang, W., Lu, J., Ma, Z., Fan, C.-W., Wei, S., Hao, W., Kilgore, J., Williams, N. S., Roth, M. G., Amatruda, J. F., Chen, C., \& Lum, L. (2009). Small molecule-mediated disruption of Wnt-dependent signaling in tissue regeneration and cancer. Nature Chemical Biology, 5(2), 100-107.

Chen, C.-A., Wang, T.-Y., Varadharaj, S., Reyes, L. A., Hemann, C., Talukder, M. A. H., Chen, Y.-R., Druhan, L. J., \& Zweier, J. L. (2010). S-glutathionylation uncouples eNOS and regulates its cellular and vascular function. Nature, 468(7327), 1115-1118.

Cho, S. W., Park, J.-S., Heo, H. J., Park, S.-W., Song, S., Kim, I., Han, Y.-M., Yamashita, J. K., Youm, J. B., Han, J., \& Koh, G. Y. (2014). Dual modulation of the mitochondrial permeability transition 
pore and redox signaling synergistically promotes cardiomyocyte differentiation from pluripotent stem cells. Journal of the American Heart Association, 3(2), e000693.

Cho, Y. M., Kwon, S., Pak, Y. K., Seol, H. W., Choi, Y. M., Park, D. J., Park, K. S., \& Lee, H. K. (2006). Dynamic changes in mitochondrial biogenesis and antioxidant enzymes during the spontaneous differentiation of human embryonic stem cells. Biochemical and Biophysical Research Communications, 348(4), 1472-1478.

Choi, H., Kim, S., Mukhopadhyay, P., Cho, S., Woo, J., Storz, G., \& Ryu, S. E. (2001). Structural basis of the redox switch in the OxyR transcription factor. Cell, 105(1), 103-113.

Christalla, P., Hudson, J. E., \& Zimmermann, W.-H. (2012). The cardiogenic niche as a fundamental building block of engineered myocardium. Cells, Tissues, Organs, 195(1-2), 82-93.

Christian, M., Cermak, T., Doyle, E. L., Schmidt, C., Zhang, F., Hummel, A., Bogdanove, A. J., \& Voytas, D. F. (2010). Targeting DNA double-strand breaks with TAL effector nucleases. Genetics, 186(2), 757-761.

Cong, L., Ran, F. A., Cox, D., Lin, S., Barretto, R., Habib, N., Hsu, P. D., Wu, X., Jiang, W., Marraffini, L. A., \& Zhang, F. (2013). Multiplex Genome Engineering Using CRISPR/Cas Systems. Science (New York, N.Y.), 339(6121), 819-823.

Correia, C., Koshkin, A., Duarte, P., Hu, D., Teixeira, A., Domian, I., Serra, M., \& Alves, P. M. (2017). Distinct carbon sources affect structural and functional maturation of cardiomyocytes derived from human pluripotent stem cells. Scientific Reports, 7(1), 8590.

Crompton, M. (1999). The mitochondrial permeability transition pore and its role in cell death. The Biochemical Journal, 341 ( Pt 2), 233-249.

Cyganek, L., Tiburcy, M., Sekeres, K., Gerstenberg, K., Bohnenberger, H., Lenz, C., Henze, S., Stauske, M., Salinas, G., Zimmermann, W.-H., Hasenfuss, G., \& Guan, K. (2018). Deep phenotyping of human induced pluripotent stem cell-derived atrial and ventricular cardiomyocytes. $\mathrm{JCl}$ Insight, 3(12). doi:10.1172/jci.insight.99941

Dauchet, L., Amouyel, P., \& Dallongeville, J. (2009). Fruits, vegetables and coronary heart disease. Nature Reviews. Cardiology, 6(9), 599-608.

de Jong, J. W., Schoemaker, R. G., de Jonge, R., Bernocchi, P., Keijzer, E., Harrison, R., Sharma, H. S., \& Ceconi, C. (2000). Enhanced expression and activity of xanthine oxidoreductase in the failing heart. Journal of Molecular and Cellular Cardiology, 32(11), 2083-2089.

Dębski, D., Smulik, R., Zielonka, J., Michałowski, B., Jakubowska, M., Dębowska, K., Adamus, J., Marcinek, A., Kalyanaraman, B., \& Sikora, A. (2016). Mechanism of oxidative conversion of Amplex ${ }^{\circledast}$ Red to resorufin: Pulse radiolysis and enzymatic studies. Free Radical Biology \& Medicine, 95, 323-332. 
Deshwal, S., Di Sante, M., Di Lisa, F., \& Kaludercic, N. (2017). Emerging role of monoamine oxidase as a therapeutic target for cardiovascular disease. Current Opinion in Pharmacology, 33, 64-69.

Devalla, H. D., Schwach, V., Ford, J. W., Milnes, J. T., El-Haou, S., Jackson, C., Gkatzis, K., Elliott, D. A., Chuva de Sousa Lopes, S. M., Mummery, C. L., Verkerk, A. O., \& Passier, R. (2015). Atrial-like cardiomyocytes from human pluripotent stem cells are a robust preclinical model for assessing atrial-selective pharmacology. EMBO Molecular Medicine, 7(4), 394-410.

Dey, S., DeMazumder, D., Sidor, A., Foster, D. B., \& O'Rourke, B. (2018). Mitochondrial ROS Drive Sudden Cardiac Death and Chronic Proteome Remodeling in Heart Failure. Circulation Research, CIRCRESAHA.118.312708.

Ding, L., Liang, X.-G., Hu, Y., Zhu, D.-Y., \& Lou, Y.-J. (2008). Involvement of p38MAPK and reactive oxygen species in icariin-induced cardiomyocyte differentiation of murine embryonic stem cells in vitro. Stem Cells and Development, 17(4), 751-760.

Eder, A., Hansen, A., Uebeler, J., Schulze, T., Neuber, C., Schaaf, S., Yuan, L., Christ, T., Vos, M. A., \& Eschenhagen, T. (2014). Effects of proarrhythmic drugs on relaxation time and beating pattern in rat engineered heart tissue. Basic Research in Cardiology, 109(6), 436.

Erickson, J. R., Joiner, M. A., Guan, X., Kutschke, W., Yang, J., Oddis, C. V., Bartlett, R. K., Lowe, J. S., O’Donnell, S. E., Aykin-Burns, N., Zimmerman, M. C., Zimmerman, K., Ham, A.-J. L., Weiss, R. M., Spitz, D. R., Shea, M. A., Colbran, R. J., Mohler, P. J., \& Anderson, M. E. (2008). A dynamic pathway for calcium-independent activation of CaMKII by methionine oxidation. Cell, 133(3), 462-474.

Ermakova, Y. G., Bilan, D. S., Matlashov, M. E., Mishina, N. M., Markvicheva, K. N., Subach, O. M., Subach, F. V., Bogeski, I., Hoth, M., Enikolopov, G., \& Belousov, V. V. (2014). Red fluorescent genetically encoded indicator for intracellular hydrogen peroxide. Nature Communications, 5, 5222 .

Farboud, B., Jarvis, E., Roth, T. L., Shin, J., Corn, J. E., Marson, A., Meyer, B. J., Patel, N. H., \& Hochstrasser, M. L. (2018). Enhanced Genome Editing with Cas9 Ribonucleoprotein in Diverse Cells and Organisms. Journal of Visualized Experiments : JoVE, (135). doi:10.3791/57350

Gherghiceanu, M., Barad, L., Novak, A., Reiter, I., Itskovitz-Eldor, J., Binah, O., \& Popescu, L. M. (2011). Cardiomyocytes derived from human embryonic and induced pluripotent stem cells: comparative ultrastructure. Journal of Cellular and Molecular Medicine, 15(11), 2539-2551.

Girard, J., Ferré, P., Pégorier, J. P., \& Duée, P. H. (1992). Adaptations of glucose and fatty acid metabolism during perinatal period and suckling-weaning transition. Physiological Reviews, 72(2), 507-562. 
Givertz, M. M., Anstrom, K. J., Redfield, M. M., Deswal, A., Haddad, H., Butler, J., Tang, W. H. W., Dunlap, M. E., LeWinter, M. M., Mann, D. L., Felker, G. M., O’Connor, C. M., Goldsmith, S. R., Ofili, E. O., Saltzberg, M. T., Margulies, K. B., Cappola, T. P., Konstam, M. A., Semigran, M. J., McNulty, S. E., Lee, K. L., Shah, M. R., Hernandez, A. F., \& NHLBI Heart Failure Clinical Research Network. (2015). Effects of Xanthine Oxidase Inhibition in Hyperuricemic Heart Failure Patients: The Xanthine Oxidase Inhibition for Hyperuricemic Heart Failure Patients (EXACT-HF) Study. Circulation, 131(20), 1763-1771.

Godier-Furnémont, A. F. G., Tiburcy, M., Wagner, E., Dewenter, M., Lämmle, S., El-Armouche, A., Lehnart, S. E., Vunjak-Novakovic, G., \& Zimmermann, W.-H. (2015). Physiologic forcefrequency response in engineered heart muscle by electromechanical stimulation. Biomaterials, 60, 82-91.

Gonzalez, D. R., Treuer, A. V., Castellanos, J., Dulce, R. A., \& Hare, J. M. (2010). Impaired Snitrosylation of the ryanodine receptor caused by xanthine oxidase activity contributes to calcium leak in heart failure. The Journal of Biological Chemistry, 285(37), 28938-28945.

Guo, Y., Jones, W. K., Xuan, Y. T., Tang, X. L., Bao, W., Wu, W. J., Han, H., Laubach, V. E., Ping, P., Yang, Z., Qiu, Y., \& Bolli, R. (1999). The late phase of ischemic preconditioning is abrogated by targeted disruption of the inducible NO synthase gene. Proceedings of the National Academy of Sciences of the United States of America, 96(20), 11507-11512.

Gutscher, M., Pauleau, A.-L., Marty, L., Brach, T., Wabnitz, G. H., Samstag, Y., Meyer, A. J., \& Dick, T. P. (2008). Real-time imaging of the intracellular glutathione redox potential. Nature Methods, 5(6), 553-559.

Gutscher, M., Sobotta, M. C., Wabnitz, G. H., Ballikaya, S., Meyer, A. J., Samstag, Y., \& Dick, T. P. (2009). Proximity-based Protein Thiol Oxidation by H2O2-scavenging Peroxidases. Journal of Biological Chemistry, 284(46), 31532-31540.

Haapaniemi, E., Botla, S., Persson, J., Schmierer, B., \& Taipale, J. (2018). CRISPR-Cas9 genome editing induces a p53-mediated DNA damage response. Nature Medicine, 24(7), 927-930.

Hanson, G. T., Aggeler, R., Oglesbee, D., Cannon, M., Capaldi, R. A., Tsien, R. Y., \& Remington, S. J. (2004). Investigating Mitochondrial Redox Potential with Redox-sensitive Green Fluorescent Protein Indicators. Journal of Biological Chemistry, 279(13), 13044-13053.

Heim, R., Cubitt, A. B., \& Tsien, R. Y. (1995). Improved green fluorescence. Nature, 373(6516), 663664.

Heta, E. (2017, May 29). Development of a genetically encoded model for the sensing of glutathione redox potential in human embryonic stem cell-derived cardiomyocytes and fibroblasts. Retrieved from https://ediss.uni-goettingen.de/handle/11858/00-1735-0000-0023-3E5D-1 
Hotta, Y., Uchiyama, K., Takagi, T., Kashiwagi, S., Nakano, T., Mukai, R., Toyokawa, Y., Yasuda, T., Ueda, T., Suyama, Y., Murakami, T., Tanaka, M., Majima, A., Doi, T., Hirai, Y., Mizushima, K., Morita, M., Higashimura, Y., Inoue, K., Fukui, A., Okayama, T., Katada, K., Kamada, K., Handa, O., Ishikawa, T., Naito, Y., \& Itoh, Y. (2018). Transforming growth factor $\beta 1$-induced collagen production in myofibroblasts is mediated by reactive oxygen species derived from NADPH oxidase 4. Biochemical and Biophysical Research Communications, 506(3), 557-562.

Hu, D., Linders, A., Yamak, A., Correia, C., Kijlstra, J. D., Garakani, A., Xiao, L., Milan, D. J., van der Meer, P., Serra, M., Alves, P. M., \& Domian, I. J. (2018). Metabolic Maturation of Human Pluripotent Stem Cell-Derived Cardiomyocytes by Inhibition of HIF1 $\alpha$ and LDHA. Circulation Research, 123(9), 1066-1079.

Humphries, K. M., Deal, M. S., \& Taylor, S. S. (2005). Enhanced dephosphorylation of cAMPdependent protein kinase by oxidation and thiol modification. The Journal of Biological Chemistry, 280(4), 2750-2758.

Ihry, R. J., Worringer, K. A., Salick, M. R., Frias, E., Ho, D., Theriault, K., Kommineni, S., Chen, J., Sondey, M., Ye, C., Randhawa, R., Kulkarni, T., Yang, Z., McAllister, G., Russ, C., Reece-Hoyes, J., Forrester, W., Hoffman, G. R., Dolmetsch, R., \& Kaykas, A. (2018). p53 inhibits CRISPRCas9 engineering in human pluripotent stem cells. Nature Medicine, 24(7), 939-946.

Jacob, F., Yonis, A. Y., Cuello, F., Luther, P., Schulze, T., Eder, A., Streichert, T., Mannhardt, I., Hirt, M. N., Schaaf, S., Stenzig, J., Force, T., Eschenhagen, T., \& Hansen, A. (2016). Analysis of Tyrosine Kinase Inhibitor-Mediated Decline in Contractile Force in Rat Engineered Heart Tissue. PloS One, 11(2), e0145937.

Jinek, M., Chylinski, K., Fonfara, I., Hauer, M., Doudna, J. A., \& Charpentier, E. (2012). A programmable dual-RNA-guided DNA endonuclease in adaptive bacterial immunity. Science (New York, N.Y.), 337(6096), 816-821.

Kaludercic, N., Mialet-Perez, J., Paolocci, N., Parini, A., \& Di Lisa, F. (2014). Monoamine oxidases as sources of oxidants in the heart. Journal of Molecular and Cellular Cardiology, 73, 34-42.

Kim, J.-S., Jin, Y., \& Lemasters, J. J. (2006). Reactive oxygen species, but not Ca2+ overloading, trigger $\mathrm{pH}$ - and mitochondrial permeability transition-dependent death of adult rat myocytes after ischemia-reperfusion. American Journal of Physiology. Heart and Circulatory Physiology, 290(5), H2024-2034.

Kroll, K., Chabria, M., Wang, K., Häusermann, F., Schuler, F., \& Polonchuk, L. (2017). Electromechanical conditioning of human iPSC-derived cardiomyocytes for translational research. Progress in Biophysics and Molecular Biology, 130(Pt B), 212-222. 
La Russa, M. F., \& Qi, L. S. (2015). The New State of the Art: Cas9 for Gene Activation and Repression. Molecular and Cellular Biology, 35(22), 3800-3809.

Laksman, Z., Wauchop, M., Lin, E., Protze, S., Lee, J., Yang, W., Izaddoustdar, F., Shafaattalab, S., Gepstein, L., Tibbits, G. F., Keller, G., \& Backx, P. H. (2017). Modeling Atrial Fibrillation using Human Embryonic Stem Cell-Derived Atrial Tissue. Scientific Reports, 7(1), 5268.

Lee, J. H., Protze, S. I., Laksman, Z., Backx, P. H., \& Keller, G. M. (2017). Human Pluripotent Stem CellDerived Atrial and Ventricular Cardiomyocytes Develop from Distinct Mesoderm Populations. Cell Stem Cell, 21(2), 179-194.e4.

Lesnefsky, E. J., Chen, Q., Tandler, B., \& Hoppel, C. L. (2017). Mitochondrial Dysfunction and Myocardial Ischemia-Reperfusion: Implications for Novel Therapies. Annual Review of Pharmacology and Toxicology, 57, 535-565.

Li, Y., Asfour, H., \& Bursac, N. (2017). Age-dependent functional crosstalk between cardiac fibroblasts and cardiomyocytes in a 3D engineered cardiac tissue. Acta Biomaterialia, 55, 120-130.

Lian, X., Zhang, J., Azarin, S. M., Zhu, K., Hazeltine, L. B., Bao, X., Hsiao, C., Kamp, T. J., \& Palecek, S. P. (2013). Directed cardiomyocyte differentiation from human pluripotent stem cells by modulating Wnt/ $\beta$-catenin signaling under fully defined conditions. Nature Protocols, 8(1), 162-175.

Long, C., Li, H., Tiburcy, M., Rodriguez-Caycedo, C., Kyrychenko, V., Zhou, H., Zhang, Y., Min, Y.-L., Shelton, J. M., Mammen, P. P. A., Liaw, N. Y., Zimmermann, W.-H., Bassel-Duby, R., Schneider, J. W., \& Olson, E. N. (2018). Correction of diverse muscular dystrophy mutations in human engineered heart muscle by single-site genome editing. Science Advances, 4(1), eaap9004.

Looi, Y. H., Grieve, D. J., Siva, A., Walker, S. J., Anilkumar, N., Cave, A. C., Marber, M., Monaghan, M. J., \& Shah, A. M. (2008). Involvement of Nox2 NADPH oxidase in adverse cardiac remodeling after myocardial infarction. Hypertension (Dallas, Tex.: 1979), 51(2), 319-325.

Lopaschuk, G. D., \& Jaswal, J. S. (2010). Energy Metabolic Phenotype of the Cardiomyocyte During Development, Differentiation, and Postnatal Maturation. Journal of Cardiovascular Pharmacology, 56(2), 130.

Lundy, S. D., Zhu, W.-Z., Regnier, M., \& Laflamme, M. A. (2013). Structural and functional maturation of cardiomyocytes derived from human pluripotent stem cells. Stem Cells and Development, 22(14), 1991-2002.

Makinde, A. O., Kantor, P. F., \& Lopaschuk, G. D. (1998). Maturation of fatty acid and carbohydrate metabolism in the newborn heart. Molecular and Cellular Biochemistry, 188(1-2), 49-56. 
Mali, P., Yang, L., Esvelt, K. M., Aach, J., Guell, M., DiCarlo, J. E., Norville, J. E., \& Church, G. M. (2013). RNA-Guided Human Genome Engineering via Cas9. Science (New York, N.Y.), 339(6121), 823-826.

Mandal, S., Lindgren, A. G., Srivastava, A. S., Clark, A. T., \& Banerjee, U. (2011). Mitochondrial Function Controls Proliferation and Early Differentiation Potential of Embryonic Stem Cells. STEM CELLS, 29(3), 486-495.

Marchesi, E., Rota, C., Fann, Y. C., Chignell, C. F., \& Mason, R. P. (1999). Photoreduction of the fluorescent dye 2'-7'-dichlorofluorescein: a spin trapping and direct electron spin resonance study with implications for oxidative stress measurements. Free Radical Biology \& Medicine, 26(1-2), 148-161.

Markvicheva, K. N., Bilan, D. S., Mishina, N. M., Gorokhovatsky, A. Y., Vinokurov, L. M., Lukyanov, S., \& Belousov, V. V. (2011). A genetically encoded sensor for $\mathrm{H} 2 \mathrm{O} 2$ with expanded dynamic range. Bioorganic \& Medicinal Chemistry, 19(3), 1079-1084.

Matlashov, M. E., Belousov, V. V., \& Enikolopov, G. (2014). How Much H2O2 Is Produced by Recombinant D-Amino Acid Oxidase in Mammalian Cells? Antioxidants \& Redox Signaling, 20(7), 1039-1044.

Matlashov, M. E., Bogdanova, Y. A., Ermakova, G. V., Mishina, N. M., Ermakova, Y. G., Nikitin, E. S., Balaban, P. M., Okabe, S., Lukyanov, S., Enikolopov, G., Zaraisky, A. G., \& Belousov, V. V. (2015). Fluorescent ratiometric pH indicator SypHer2: Applications in neuroscience and regenerative biology. Biochimica Et Biophysica Acta, 1850(11), 2318-2328.

Meyer, A. J., \& Dick, T. P. (2010). Fluorescent protein-based redox probes. Antioxidants \& Redox Signaling, 13(5), 621-650.

Meyer, T., Tiburcy, M., \& Zimmermann, W.-H. (2019). Cardiac macrotissues-on-a-plate models for phenotypic drug screens. Advanced Drug Delivery Reviews. doi:10.1016/j.addr.2019.03.002

Miller, J. C., Tan, S., Qiao, G., Barlow, K. A., Wang, J., Xia, D. F., Meng, X., Paschon, D. E., Leung, E., Hinkley, S. J., Dulay, G. P., Hua, K. L., Ankoudinova, I., Cost, G. J., Urnov, F. D., Zhang, H. S., Holmes, M. C., Zhang, L., Gregory, P. D., \& Rebar, E. J. (2011). A TALE nuclease architecture for efficient genome editing. Nature Biotechnology, 29(2), 143-148.

Mills, R. J., Titmarsh, D. M., Koenig, X., Parker, B. L., Ryall, J. G., Quaife-Ryan, G. A., Voges, H. K., Hodson, M. P., Ferguson, C., Drowley, L., Plowright, A. T., Needham, E. J., Wang, Q.-D., Gregorevic, P., Xin, M., Thomas, W. G., Parton, R. G., Nielsen, L. K., Launikonis, B. S., James, D. E., Elliott, D. A., Porrello, E. R., \& Hudson, J. E. (2017). Functional screening in human cardiac organoids reveals a metabolic mechanism for cardiomyocyte cell cycle arrest. Proceedings of the National Academy of Sciences, 114(40), E8372-E8381. 
Miwa, S., Treumann, A., Bell, A., Vistoli, G., Nelson, G., Hay, S., \& von Zglinicki, T. (2016).

Carboxylesterase converts Amplex red to resorufin: Implications for mitochondrial H2O2 release assays. Free Radical Biology \& Medicine, 90, 173-183.

Morgan, B., Van Laer, K., Owusu, T. N. E., Ezerina, D., Pastor-Flores, D., Amponsah, P. S., Tursch, A., \& Dick, T. P. (2016). Real-time monitoring of basal H2O2 levels with peroxiredoxin-based probes. Nature Chemical Biology, 12(6), 437-443.

Murphy, M. P. (2009). How mitochondria produce reactive oxygen species. The Biochemical Journal, 417(1), 1-13.

Mussolino, C., Morbitzer, R., Lütge, F., Dannemann, N., Lahaye, T., \& Cathomen, T. (2011). A novel TALE nuclease scaffold enables high genome editing activity in combination with low toxicity. Nucleic Acids Research, 39(21), 9283-9293.

Myung, S.-K., Ju, W., Cho, B., Oh, S.-W., Park, S. M., Koo, B.-K., \& Park, B.-J. (2013). Efficacy of vitamin and antioxidant supplements in prevention of cardiovascular disease: systematic review and meta-analysis of randomised controlled trials. BMJ, 346, f10.

Na, L., Wartenberg, M., Nau, H., Hescheler, J., \& Sauer, H. (2003). Anticonvulsant valproic acid inhibits cardiomyocyte differentiation of embryonic stem cells by increasing intracellular levels of reactive oxygen species. Birth Defects Research. Part A, Clinical and Molecular Teratology, 67(3), 174-180.

Naito, H., Melnychenko, I., Didié, M., Schneiderbanger, K., Schubert, P., Rosenkranz, S., Eschenhagen, T., \& Zimmermann, W.-H. (2006). Optimizing engineered heart tissue for therapeutic applications as surrogate heart muscle. Circulation, 114(1 Suppl), 172-78.

Naumova, A. V., Chacko, V. P., Ouwerkerk, R., Stull, L., Marbán, E., \& Weiss, R. G. (2006). Xanthine oxidase inhibitors improve energetics and function after infarction in failing mouse hearts. American Journal of Physiology. Heart and Circulatory Physiology, 290(2), H837-843.

Nott, A., Watson, P. M., Robinson, J. D., Crepaldi, L., \& Riccio, A. (2008). S-Nitrosylation of histone deacetylase 2 induces chromatin remodelling in neurons. Nature, 455(7211), 411-415.

Nunes, S. S., Miklas, J. W., Liu, J., Aschar-Sobbi, R., Xiao, Y., Zhang, B., Jiang, J., Massé, S., Gagliardi, M., Hsieh, A., Thavandiran, N., Laflamme, M. A., Nanthakumar, K., Gross, G. J., Backx, P. H., Keller, G., \& Radisic, M. (2013). Biowire: a platform for maturation of human pluripotent stem cell-derived cardiomyocytes. Nature Methods, 10(8), 781-787.

Oceguera-Yanez, F., Kim, S.-I., Matsumoto, T., Tan, G. W., Xiang, L., Hatani, T., Kondo, T., Ikeya, M., Yoshida, Y., Inoue, H., \& Woltjen, K. (2016). Engineering the AAVS1 locus for consistent and scalable transgene expression in human iPSCs and their differentiated derivatives. Methods, 101, 43-55. 
Panieri, E., Millia, C., \& Santoro, M. M. (2017). Real-time quantification of subcellular H2O2 and glutathione redox potential in living cardiovascular tissues. Free Radical Biology \& Medicine, 109, 189-200.

Paradis, S., Charles, A.-L., Meyer, A., Lejay, A., Scholey, J. W., Chakfé, N., Zoll, J., \& Geny, B. (2016). Chronology of mitochondrial and cellular events during skeletal muscle ischemiareperfusion. American Journal of Physiology. Cell Physiology, 310(11), C968-982.

Parikh, S. S., Blackwell, D. J., Gomez-Hurtado, N., Frisk, M., Wang, L., Kim, K., Dahl, C. P., Fiane, A., Tønnessen, T., Kryshtal, D. O., Louch, W. E., \& Knollmann, B. C. (2017). Thyroid and Glucocorticoid Hormones Promote Functional T-Tubule Development in Human-Induced Pluripotent Stem Cell-Derived Cardiomyocytes. Circulation Research, 121(12), 1323-1330.

Radisic, M., Park, H., Shing, H., Consi, T., Schoen, F. J., Langer, R., Freed, L. E., \& Vunjak-Novakovic, G. (2004). Functional assembly of engineered myocardium by electrical stimulation of cardiac myocytes cultured on scaffolds. Proceedings of the National Academy of Sciences of the United States of America, 101(52), 18129-18134.

Ramos, C., Brito, R., González-Montero, J., Valls, N., Gormaz, J. G., Prieto, J. C., Aguayo, R., Puentes, Á., Noriega, V., Pereira, G., Palavecino, T., \& Rodrigo, R. (2017). Effects of a novel ascorbatebased protocol on infarct size and ventricle function in acute myocardial infarction patients undergoing percutaneous coronary angioplasty. Archives of Medical Science: AMS, 13(3), 558-567.

Rezende, F., Brandes, R. P., \& Schröder, K. (2018). Detection of Hydrogen Peroxide with Fluorescent Dyes. Antioxidants \& Redox Signaling, 29(6), 585-602.

Riegler, J., Tiburcy, M., Ebert, A., Tzatzalos, E., Raaz, U., Abilez, O. J., Shen, Q., Kooreman, N. G., Neofytou, E., Chen, V. C., Wang, M., Meyer, T., Tsao, P. S., Connolly, A. J., Couture, L. A., Gold, J. D., Zimmermann, W. H., \& Wu, J. C. (2015). Human Engineered Heart Muscles Engraft and Survive Long Term in a Rodent Myocardial Infarction Model. Circulation Research, 117(8), 720-730.

Robinson, K. M., Janes, M. S., Pehar, M., Monette, J. S., Ross, M. F., Hagen, T. M., Murphy, M. P., \& Beckman, J. S. (2006). Selective fluorescent imaging of superoxide in vivo using ethidiumbased probes. Proceedings of the National Academy of Sciences of the United States of America, 103(41), 15038-15043.

Ronaldson-Bouchard, K., Ma, S. P., Yeager, K., Chen, T., Song, L., Sirabella, D., Morikawa, K., Teles, D., Yazawa, M., \& Vunjak-Novakovic, G. (2018). Advanced maturation of human cardiac tissue grown from pluripotent stem cells. Nature, 556(7700), 239-243. 
Santos, C. X. C., Raza, S., \& Shah, A. M. (2016). Redox signaling in the cardiomyocyte: From physiology to failure. The International Journal of Biochemistry \& Cell Biology, 74, 145-151.

Sauer, H., Neukirchen, W., Rahimi, G., Grünheck, F., Hescheler, J., \& Wartenberg, M. (2004). Involvement of reactive oxygen species in cardiotrophin-1-induced proliferation of cardiomyocytes differentiated from murine embryonic stem cells. Experimental Cell Research, 294(2), 313-324.

Sauer, H., Rahimi, G., Hescheler, J., \& Wartenberg, M. (2000). Role of reactive oxygen species and phosphatidylinositol 3-kinase in cardiomyocyte differentiation of embryonic stem cells. FEBS Letters, 476(3), 218-223.

Schlick, S. F. (2019, February 1). Fibroblast-Cardiomyocyte Cross-Talk in Heart Muscle Formation and Function. Retrieved from https://ediss.uni-goettingen.de/handle/11858/00-1735-0000002E-E57D-3

Schlick, S. F., Spreckelsen, F., Tiburcy, M., Iyer, L. M., Meyer, T., Zelarayan, L. C., Luther, S., Parlitz, U., Zimmermann, W.-H., \& Rehfeldt, F. (2018). Agonistic and antagonistic roles of fibroblasts and cardiomyocytes on viscoelastic stiffening of engineered human myocardium. Progress in Biophysics and Molecular Biology. doi:10.1016/j.pbiomolbio.2018.11.011

Shackelford, R. E., Heinloth, A. N., Heard, S. C., \& Paules, R. S. (2005). Cellular and molecular targets of protein S-glutathiolation. Antioxidants \& Redox Signaling, 7(7-8), 940-950.

Shao, D., Oka, S., Brady, C. D., Haendeler, J., Eaton, P., \& Sadoshima, J. (2012). Redox modification of cell signaling in the cardiovascular system. Journal of Molecular and Cellular Cardiology, 52(3), 550-558.

Sharifpanah, F., Wartenberg, M., Hannig, M., Piper, H.-M., \& Sauer, H. (2008). Peroxisome proliferator-activated receptor alpha agonists enhance cardiomyogenesis of mouse ES cells by utilization of a reactive oxygen species-dependent mechanism. Stem Cells (Dayton, Ohio), 26(1), 64-71.

Shen, W.-L., Gao, P.-J., Che, Z.-Q., Ji, K.-D., Yin, M., Yan, C., Berk, B. C., \& Zhu, D.-L. (2006). NAD(P)H oxidase-derived reactive oxygen species regulate angiotensin-II induced adventitial fibroblast phenotypic differentiation. Biochemical and Biophysical Research Communications, 339(1), 337-343.

Sies, H. (2017). Hydrogen peroxide as a central redox signaling molecule in physiological oxidative stress: Oxidative eustress. Redox Biology, 11, 613-619.

Soong, P. L., Tiburcy, M., \& Zimmermann, W.-H. (2012). Cardiac differentiation of human embryonic stem cells and their assembly into engineered heart muscle. Current Protocols in Cell Biology /Editorial Board, Juan S. Bonifacino ... [et Al.], Chapter 23, Unit23.8. 
Später, D., Hansson, E. M., Zangi, L., \& Chien, K. R. (2014). How to make a cardiomyocyte. Development, 141(23), 4418-4431.

Stadtman, E. R., Moskovitz, J., \& Levine, R. L. (2003). Oxidation of methionine residues of proteins: biological consequences. Antioxidants \& Redox Signaling, 5(5), 577-582.

Steinhorn, B., Sartoretto, J. L., Sorrentino, A., Romero, N., Kalwa, H., Abel, E. D., \& Michel, T. (2017). Insulin-dependent metabolic and inotropic responses in the heart are modulated by hydrogen peroxide from NADPH-oxidase isoforms NOX2 and NOX4. Free Radical Biology and Medicine, 113, 16-25.

Streckfuss-Bömeke, K., Tiburcy, M., Fomin, A., Luo, X., Li, W., Fischer, C., Özcelik, C., Perrot, A., Sossalla, S., Haas, J., Vidal, R. O., Rebs, S., Khadjeh, S., Meder, B., Bonn, S., Linke, W. A., Zimmermann, W.-H., Hasenfuss, G., \& Guan, K. (2017). Severe DCM phenotype of patient harboring RBM20 mutation S635A can be modeled by patient-specific induced pluripotent stem cell-derived cardiomyocytes. Journal of Molecular and Cellular Cardiology, 113, 9-21.

Sur, S. (2016, July 12). Control of cardiogenesis and homeostasis by cardiac fibroblasts. Retrieved from https://ediss.uni-goettingen.de/handle/11858/00-1735-0000-0028-87B6-9

Swain, L., Kesemeyer, A., Meyer-Roxlau, S., Vettel, C., Zieseniss, A., Güntsch, A., Jatho, A., Becker, A., Nanadikar, M. S., Morgan, B., Dennerlein, S., Shah, A. M., El-Armouche, A., Nikolaev, V. O., \& Katschinski, D. M. (2016). Redox Imaging Using Cardiac Myocyte Specific Transgenic Biosensor Mice. Circulation Research, CIRCRESAHA.116.309551.

Takahashi, K., Tanabe, K., Ohnuki, M., Narita, M., Ichisaka, T., Tomoda, K., \& Yamanaka, S. (2007). Induction of pluripotent stem cells from adult human fibroblasts by defined factors. Cell, 131(5), 861-872.

Takahashi, K., \& Yamanaka, S. (2006). Induction of Pluripotent Stem Cells from Mouse Embryonic and Adult Fibroblast Cultures by Defined Factors. Cell, 126(4), 663-676.

Takahashi, T., Lord, B., Schulze, P. C., Fryer, R. M., Sarang, S. S., Gullans, S. R., \& Lee, R. T. (2003). Ascorbic acid enhances differentiation of embryonic stem cells into cardiac myocytes. Circulation, 107(14), 1912-1916.

Thomson, J. A., Itskovitz-Eldor, J., Shapiro, S. S., Waknitz, M. A., Swiergiel, J. J., Marshall, V. S., \& Jones, J. M. (1998). Embryonic stem cell lines derived from human blastocysts. Science (New York, N.Y.), 282(5391), 1145-1147.

Tiburcy, M., Didié, M., Boy, O., Christalla, P., Döker, S., Naito, H., Karikkineth, B. C., El-Armouche, A., Grimm, M., Nose, M., Eschenhagen, T., Zieseniss, A., Katschinksi, D. M., Hamdani, N., Linke, W. A., Yin, X., Mayr, M., \& Zimmermann, W.-H. (2011). Terminal differentiation, advanced 
organotypic maturation, and modeling of hypertrophic growth in engineered heart tissue. Circulation Research, 109(10), 1105-1114.

Tiburcy, M., Hudson, J. E., Balfanz, P., Schlick, S., Meyer, T., Liao, M.-L. C., Levent, E., Raad, F., Zeidler, S., Wingender, E., Riegler, J., Wang, M., Gold, J. D., Kehat, I., Wettwer, E., Ravens, U., Dierickx, P., Laake, L. W. van, Goumans, M. J., Khadjeh, S., Toischer, K., Hasenfuss, G., Couture, L. A., Unger, A., Linke, W. A., Araki, T., Neel, B., Keller, G., Gepstein, L., Wu, J. C., \& Zimmermann, W.-H. (2017). Defined Engineered Human Myocardium With Advanced Maturation for Applications in Heart Failure Modeling and Repair. Circulation, 135(19), 1832-1847.

Tiburcy, M., Meyer, T., Soong, P. L., \& Zimmermann, W.-H. (2014). Collagen-based engineered heart muscle. Methods in Molecular Biology (Clifton, N.J.), 1181, 167-176.

Trautsch, I., Heta, E., Soong, P. L., Levent, E., Nikolaev, V. O., Bogeski, I., Katschinski, D. M., Mayr, M., \& Zimmermann, W.-H. (2019). Optogenetic Monitoring of the Glutathione Redox State in Engineered Human Myocardium. Frontiers in Physiology, 10. doi:10.3389/fphys.2019.00272

Tsujita, K., Shimomura, H., Kaikita, K., Kawano, H., Hokamaki, J., Nagayoshi, Y., Yamashita, T., Fukuda, M., Nakamura, Y., Sakamoto, T., Yoshimura, M., \& Ogawa, H. (2006). Long-term efficacy of edaravone in patients with acute myocardial infarction. Circulation Journal: Official Journal of the Japanese Circulation Society, 70(7), 832-837.

Tu, C., Allen, A., Deng, W., Conroy, O., Nambiar, M., \& Zoldan, J. (2018). Commonly used thiolcontaining antioxidants reduce cardiac differentiation and alter gene expression ratios of sarcomeric isoforms. Experimental Cell Research, 370(1), 150-159.

Tulloch, N. L., Muskheli, V., Razumova, M. V., Korte, F. S., Regnier, M., Hauch, K. D., Pabon, L., Reinecke, H., \& Murry, C. E. (2011). Growth of engineered human myocardium with mechanical loading and vascular coculture. Circulation Research, 109(1), 47-59.

Urnov, F. D., Rebar, E. J., Holmes, M. C., Zhang, H. S., \& Gregory, P. D. (2010). Genome editing with engineered zinc finger nucleases. Nature Reviews Genetics, 11(9), 636-646.

Valls, N., Gormaz, J. G., Aguayo, R., González, J., Brito, R., Hasson, D., Libuy, M., Ramos, C., Carrasco, R., Prieto, J. C., Dussaillant, G., Puentes, Á., Noriega, V., \& Rodrigo, R. (2016). Amelioration of persistent left ventricular function impairment through increased plasma ascorbate levels following myocardial infarction. Redox Report: Communications in Free Radical Research, 21(2), 75-83.

van den Berg, C. W., Okawa, S., Chuva de Sousa Lopes, S. M., van Iperen, L., Passier, R., Braam, S. R., Tertoolen, L. G., del Sol, A., Davis, R. P., \& Mummery, C. L. (2015). Transcriptome of human 
foetal heart compared with cardiomyocytes from pluripotent stem cells. Development (Cambridge, England), 142(18), 3231-3238.

Voges, H. K., Mills, R. J., Elliott, D. A., Parton, R. G., Porrello, E. R., \& Hudson, J. E. (2017). Development of a human cardiac organoid injury model reveals innate regenerative potential. Development (Cambridge, England), 144(6), 1118-1127.

Wang, W., Fang, H., Groom, L., Cheng, A., Zhang, W., Liu, J., Wang, X., Li, K., Han, P., Zheng, M., Yin, J., Wang, W., Mattson, M. P., Kao, J. P. Y., Lakatta, E. G., Sheu, S.-S., Ouyang, K., Chen, J., Dirksen, R. T., \& Cheng, H. (2008). Superoxide flashes in single mitochondria. Cell, 134(2), 279-290.

Weinberger, F., Breckwoldt, K., Pecha, S., Kelly, A., Geertz, B., Starbatty, J., Yorgan, T., Cheng, K.-H., Lessmann, K., Stolen, T., Scherrer-Crosbie, M., Smith, G., Reichenspurner, H., Hansen, A., \& Eschenhagen, T. (2016). Cardiac repair in guinea pigs with human engineered heart tissue from induced pluripotent stem cells. Science Translational Medicine, 8(363), 363ra148.

Weng, Z., Kong, C.-W., Ren, L., Karakikes, I., Geng, L., He, J., Chow, M. Z. Y., Mok, C. F., Chan, H. Y. S., Webb, S. E., Keung, W., Chow, H., Miller, A. L., Leung, A. Y. H., Hajjar, R. J., Li, R. A., \& Chan, C. W. (2014). A simple, cost-effective but highly efficient system for deriving ventricular cardiomyocytes from human pluripotent stem cells. Stem Cells and Development, 23(14), 1704-1716.

Wong, C. M., Marcocci, L., Liu, L., \& Suzuki, Y. J. (2010). Cell signaling by protein carbonylation and decarbonylation. Antioxidants \& Redox Signaling, 12(3), 393-404.

Xia, Y., Tsai, A. L., Berka, V., \& Zweier, J. L. (1998). Superoxide generation from endothelial nitricoxide synthase. A Ca2+/calmodulin-dependent and tetrahydrobiopterin regulatory process. The Journal of Biological Chemistry, 273(40), 25804-25808.

Xu, X., \& Qi, L. S. (2019). A CRISPR-dCas Toolbox for Genetic Engineering and Synthetic Biology. Journal of Molecular Biology, 431(1), 34-47.

Yang, X., Cohen, M. V., \& Downey, J. M. (2010). Mechanism of cardioprotection by early ischemic preconditioning. Cardiovascular Drugs and Therapy, 24(3), 225-234.

Yang, X., Rodriguez, M., Pabon, L., Fischer, K. A., Reinecke, H., Regnier, M., Sniadecki, N. J., RuoholaBaker, H., \& Murry, C. E. (2014). Tri-iodo-I-thyronine promotes the maturation of human cardiomyocytes-derived from induced pluripotent stem cells. Journal of Molecular and Cellular Cardiology, 72, 296-304.

Yildirim, Y., Naito, H., Didié, M., Karikkineth, B. C., Biermann, D., Eschenhagen, T., \& Zimmermann, W.-H. (2007). Development of a biological ventricular assist device: preliminary data from a small animal model. Circulation, 116(11 Suppl), I16-23. 
Yoshida, T., Maulik, N., Ho, Y. S., Alam, J., \& Das, D. K. (2001). H(mox-1) constitutes an adaptive response to effect antioxidant cardioprotection: A study with transgenic mice heterozygous for targeted disruption of the Heme oxygenase-1 gene. Circulation, 103(12), 1695-1701.

Zhang, J., Khvorostov, I., Hong, J. S., Oktay, Y., Vergnes, L., Nuebel, E., Wahjudi, P. N., Setoguchi, K., Wang, G., Do, A., Jung, H.-J., McCaffery, J. M., Kurland, I. J., Reue, K., Lee, W.-N. P., Koehler, C. M., \& Teitell, M. A. (2011). UCP2 regulates energy metabolism and differentiation potential of human pluripotent stem cells. The EMBO Journal, 30(24), 4860-4873.

Zhao, B., Summers, F. A., \& Mason, R. P. (2012). Photooxidation of Amplex Red to resorufin: implications of exposing the Amplex Red assay to light. Free Radical Biology \& Medicine, 53(5), 1080-1087.

Zheng, M., Aslund, F., \& Storz, G. (1998). Activation of the OxyR transcription factor by reversible disulfide bond formation. Science (New York, N.Y.), 279(5357), 1718-1721.

Zhou, M., Diwu, Z., Panchuk-Voloshina, N., \& Haugland, R. P. (1997). A stable nonfluorescent derivative of resorufin for the fluorometric determination of trace hydrogen peroxide: applications in detecting the activity of phagocyte NADPH oxidase and other oxidases. Analytical Biochemistry, 253(2), 162-168.

Zhou, T., Prather, E. R., Garrison, D. E., \& Zuo, L. (2018). Interplay between ROS and Antioxidants during Ischemia-Reperfusion Injuries in Cardiac and Skeletal Muscle. International Journal of Molecular Sciences, 19(2). doi:10.3390/ijms19020417

Zielonka, J., \& Kalyanaraman, B. (2010). Hydroethidine- and Mito-SOX-derived red fluorescence is not a reliable indicator of intracellular superoxide formation: Another inconvenient truth. Free Radical Biology \& Medicine, 48(8), 983-1001.

Zimmermann, W.-H., Melnychenko, I., Wasmeier, G., Didié, M., Naito, H., Nixdorff, U., Hess, A., Budinsky, L., Brune, K., Michaelis, B., Dhein, S., Schwoerer, A., Ehmke, H., \& Eschenhagen, T. (2006). Engineered heart tissue grafts improve systolic and diastolic function in infarcted rat hearts. Nature Medicine, 12(4), 452-458.

Zimmermann, W.-H., Schneiderbanger, K., Schubert, P., Didié, M., Münzel, F., Heubach, J. F., Kostin, S., Neuhuber, W. L., \& Eschenhagen, T. (2002). Tissue engineering of a differentiated cardiac muscle construct. Circulation Research, 90(2), 223-230.

Zorov, D. B., Filburn, C. R., Klotz, L. O., Zweier, J. L., \& Sollott, S. J. (2000). Reactive oxygen species (ROS)-induced ROS release: a new phenomenon accompanying induction of the mitochondrial permeability transition in cardiac myocytes. The Journal of Experimental Medicine, 192(7), 1001-1014. 


\section{Appendix}

\subsection{List of antibodies}

Table 6 List of all antibodies used for flow cytometry (FC), immunofluorescence (IF) and Western blot (WB). ms:mouse, rb: rabbit, hm: human.

\begin{tabular}{|c|c|c|c|c|c|c|c|}
\hline & antigen/specificity & species & manufacturer & cat \# & $\begin{array}{l}\text { dilutio } \\
\text { n FC }\end{array}$ & $\begin{array}{c}\text { dilution } \\
\text { IF }\end{array}$ & $\begin{array}{c}\text { dilution } \\
\text { WB }\end{array}$ \\
\hline \multirow{8}{*}{ 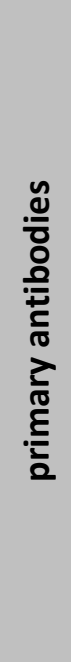 } & $\alpha$-actinin & $\mathrm{ms}$ & Sigma & A7811 & $1: 4000$ & 1:1000 & - \\
\hline & $\alpha$-tubulin & $\mathrm{ms}$ & Sigma & T5168 & - & - & 1:2000 \\
\hline & eGFP Tag & $\mathrm{rb}$ & $\begin{array}{l}\text { Thermo } \\
\text { Fisher }\end{array}$ & CAB4211 & - & $1: 100$ & $1: 500$ \\
\hline & GAPDH & $\mathrm{ms}$ & $\begin{array}{l}\text { Zytomed } \\
\text { Systems }\end{array}$ & RGM2-6C5 & - & - & $1: 100000$ \\
\hline & Nanog-PE & $\mathrm{hm}$ & $\begin{array}{l}\text { Miltenyi } \\
\text { Biotech }\end{array}$ & $\begin{array}{l}130-105- \\
050\end{array}$ & $1: 25$ & - & - \\
\hline & Oct4-Alexa-647 & $\mathrm{ms}$ & $\begin{array}{l}\text { BD } \\
\text { Biosciences }\end{array}$ & 560329 & $1: 50$ & - & - \\
\hline & $\begin{array}{l}\text { total OxPhos antibody } \\
\text { cocktail }\end{array}$ & $\mathrm{ms}$ & abcam & ab110413 & - & - & $1: 250$ \\
\hline & Tra1-60-Alexa-647 & $\mathrm{ms}$ & $\begin{array}{l}\text { BD } \\
\text { Biosciences }\end{array}$ & 560850 & $1: 50$ & - & - \\
\hline \multirow{6}{*}{ 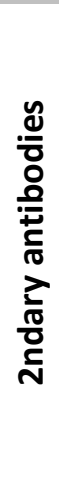 } & anti-mouse Alexa-546 & goat & $\begin{array}{l}\text { Thermo } \\
\text { Fisher }\end{array}$ & A-11003 & $1: 1000$ & 1:1000 & - \\
\hline & anti-mouse Alexa-633 & goat & $\begin{array}{l}\text { Thermo } \\
\text { Fisher }\end{array}$ & A-21052 & $1: 1000$ & 1:1000 & - \\
\hline & anti-rabbit Alexa-546 & goat & $\begin{array}{l}\text { Thermo } \\
\text { Fisher }\end{array}$ & A-11010 & $1: 1000$ & 1:1000 & - \\
\hline & anit-rabbit Alexa-633 & goat & $\begin{array}{l}\text { Thermo } \\
\text { Fisher }\end{array}$ & A-21071 & $1: 1000$ & 1:1000 & - \\
\hline & anti-mouse-HRP & goat & Dako & P0260 & - & - & $1: 10000$ \\
\hline & anti-rabbit-HRP & goat & Dako & P0448 & - & - & $1: 5000$ \\
\hline \multirow{2}{*}{$\varliminf_{0}^{\tilde{z}}$} & Hoechst 33342 & - & $\begin{array}{l}\text { Thermo } \\
\text { Fisher }\end{array}$ & H3570 & $1: 1000$ & 1:1000 & - \\
\hline & $\begin{array}{l}\text { Sytox Red dead cell } \\
\text { stain }\end{array}$ & - & $\begin{array}{l}\text { Thermo } \\
\text { Fisher }\end{array}$ & S34859 & $1: 1000$ & - & - \\
\hline
\end{tabular}

\subsection{List of primers}

Table 7 List of all primers used for cloning and genotyping. $\mathrm{T}_{\mathrm{m}}$ : melting temperature in ${ }^{\circ} \mathrm{C}$

\begin{tabular}{|c|c|c|c|}
\hline primer & sequence (5' -> 3') & $T_{m}$ & purpose \\
\hline Agel_Grx1-roGFP2_fw & $\begin{array}{l}\text { ACA CCG GTA TGG CTC AAG } \\
\text { AGT TTG TGA AC }\end{array}$ & 62 & $\begin{array}{l}\text { amplification of Grx1-roGFP2 cds, } \\
\text { Agel cut site }\end{array}$ \\
\hline $\begin{array}{l}\text { CytoGrx1roGFP2_Sal1 } \\
\text { rv }\end{array}$ & $\begin{array}{l}\text { ACAGTCGACTTACTTGTACAGCT } \\
\text { CGTCCA }\end{array}$ & 63 & $\begin{array}{l}\text { amplification of Grx1-roGFP2 cds, } \\
\text { Sall cut site }\end{array}$ \\
\hline $\begin{array}{l}\text { mitoGrx1roGFP2_Sal1 } \\
\mathrm{fw}\end{array}$ & $\begin{array}{l}\text { TTTGTCGACATGGCCTCCACTCGT } \\
\mathrm{G}\end{array}$ & 61 & $\begin{array}{l}\text { amplification of Grx1-roGFP2 cds, } \\
\text { Sall cut site }\end{array}$ \\
\hline mitoGrx1roGFP2_Sal1 & ACAGTCGACTTACTTGTACAGCT & 62 & amplification of Grx1-roGFP2 cds, \\
\hline
\end{tabular}




\begin{tabular}{|c|c|c|c|}
\hline$r v$ & CGTCCA & & Sall cut site \\
\hline $\begin{array}{l}\text { HyPer- } \\
\text { DAAO_EcoRV_fw }\end{array}$ & $\begin{array}{l}\text { GAATTCGATATCATGGAGATGGC } \\
\text { AAGCCAG }\end{array}$ & 63 & $\begin{array}{l}\text { amplification of HyPer-DAO and } \\
\text { HyPer cds, EcoRV cutsite }\end{array}$ \\
\hline HyPer-DAAO_Sall_rv & $\begin{array}{l}\text { CAGGTCGACTTAGCTCTCCCTAG } \\
\text { CTG }\end{array}$ & 63 & $\begin{array}{l}\text { amplification of HyPer-DAO cds, Sall } \\
\text { cutsite }\end{array}$ \\
\hline HyPer_Sall_rv & $\begin{array}{l}\text { ACCGTCGACTTAACCAACCGCCT } \\
\text { GTTTTAA }\end{array}$ & $\begin{array}{l}62 \\
, 5\end{array}$ & $\begin{array}{l}\text { amplification of HyPer cds, Sall } \\
\text { cutsite }\end{array}$ \\
\hline HyPer_Sbfl_rv & $\begin{array}{l}\text { GTGATTAATTAAACCAACCGCCT } \\
\text { GTTTTAA }\end{array}$ & 60 & $\begin{array}{l}\text { amplification of HyPer cds, Sbfl } \\
\text { cutsite }\end{array}$ \\
\hline AAVS1-CAG & TCTAGAGCCTCTGCTAACCATG & 62 & $\begin{array}{l}\text { sequencing of pAAVS1 donor } \\
\text { vectors }\end{array}$ \\
\hline BGH in 3-1 rev & TGACACCTACTCAGACAATG & 56 & $\begin{array}{l}\text { sequencing of pAAVS1 donor } \\
\text { vectors }\end{array}$ \\
\hline pAAVS1_EF1_rv & AAGGAGAGATGCGAGCCCCT & 62 & $\begin{array}{l}\text { sequencing of pAAVS1 donor } \\
\text { vectors }\end{array}$ \\
\hline Grx1-roGFP2_fw & TGC AAA ATC CAG CCT GGG AA & 58 & $\begin{array}{l}\text { colony PCR, sequencing of pAAVS1 } \\
\text { donor vectors }\end{array}$ \\
\hline Grx1-roGFP2_rv & CAC GAA CTC CAG CAG GAC & 58 & $\begin{array}{l}\text { colony PCR, sequencing of pAAVS1 } \\
\text { donor vectors }\end{array}$ \\
\hline mito-Grx1-roGFP2_fw & CTC AGG TCA GCA AGC GCA & 58 & $\begin{array}{l}\text { colony PCR, sequencing of pAAVS1 } \\
\text { donor vectors }\end{array}$ \\
\hline VirGrx1RoGFP_F2 & $\begin{array}{l}\text { GCTCTAGAGCAGATAGTAATCAA } \\
\text { TTACGGGGTC }\end{array}$ & 66 & $\begin{array}{l}\text { amplification of CMV-mito-Grx1- } \\
\text { roGFP2, Xbal cut site }\end{array}$ \\
\hline VirGrx1RoGFP_R3* & $\begin{array}{l}\text { ATAAGAATgcggccgcTAAACTATT } \\
\text { TACTTGTACAGCTCGTC }\end{array}$ & 66 & $\begin{array}{l}\text { amplification of CMV-mito-Grx1- } \\
\text { roGFP2, Notl cut site }\end{array}$ \\
\hline SbflAgelPacl_fw & $\begin{array}{l}\text { ATCCCTGCAGGACCGGTTTAATT } \\
\text { AAG }\end{array}$ & 63 & MCS generation, colony PCR \\
\hline SbflAgelPacl_rv & $\begin{array}{l}\text { TGGACTTAATTAAACCGGTCCTG } \\
\text { CAGGGAT }\end{array}$ & 66 & MCS generation, colony PCR \\
\hline mutEF1_fw & AGATCTgcggccgcaaggat & 62 & $\begin{array}{l}\text { mutagenesis PCR for puro } \\
\text { correction }\end{array}$ \\
\hline mutEf1_rv & GTGGCGTCTAGCGTAGGC & 60 & $\begin{array}{l}\text { mutagenesis PCR for puro } \\
\text { correction }\end{array}$ \\
\hline mutPuro_fw & GGCCCTatgaccgagtacaa & 60 & $\begin{array}{l}\text { mutagenesis PCR for puro } \\
\text { correction }\end{array}$ \\
\hline mutPuro_rv & $\begin{array}{l}\text { TAAACAAGTTAACTGATACAAAG } \\
\text { GCAT T }\end{array}$ & 61 & $\begin{array}{l}\text { mutagenesis PCR for puro } \\
\text { correction }\end{array}$ \\
\hline overlap EF1rv_puro & $\begin{array}{l}\text { TTG TAC TCG GTC ATA GGG } \\
\text { CCG TGG CGT CTA GCG TAG GC }\end{array}$ & 82 & $\begin{array}{l}\text { mutagenesis PCR for puro } \\
\text { correction }\end{array}$ \\
\hline overlap EF1_purofw & $\begin{array}{l}\text { GCC TAC GCT AGA CGC CAC } \\
\text { GGC CCT ATG ACC GAG TAC AA }\end{array}$ & 82 & $\begin{array}{l}\text { mutagenesis PCR for puro } \\
\text { correction }\end{array}$ \\
\hline gen_Grx1-roGFP2_fw & GCCCGACAACCACTACCTGAG & 62 & genotyping PCR \\
\hline gen_Grx1-roGFP2_rv & CTGGCAACTAGAAGGCACAGTC & 62 & genotyping PCR \\
\hline $\begin{array}{l}\text { gen_mito-Grx1- } \\
\text { roGFP2_fw }\end{array}$ & CTCAGGTCAGCAAGCGCA & 60 & genotyping PCR \\
\hline $\begin{array}{l}\text { gen_mito-Grx1- } \\
\text { roGFP2_rv }\end{array}$ & TAAAGACTCGAGGCACCGTT & 60 & genotyping PCR \\
\hline gen_HyPer_fw & GGTTTATCGTCCTGGCTCACC & 61 & genotyping PCR \\
\hline gen_Hyper_rv & CTAGAGAGCCCGATGACTCCG & 61 & genotyping PCR \\
\hline gen_HyPer-DAO_fw & CATAACGTTGGCCTGAGACCC & 61 & genotyping PCR \\
\hline gen_HyPer-DAO_rv & CTGGCAACTAGAAGGCACAGTC & 61 & genotyping PCR \\
\hline
\end{tabular}




\begin{tabular}{llll}
\hline AAVS1_fw & GCC CAC TGT TTC CCC TTC & 58 & genotyping PCR, wildtype locus \\
AAVS1_rV & CCAAAAGGTCAGCCTGGTAG & 60 & genotyping PCR, wildtype locus \\
AAVS1_LUKI_L_F1 & CCGGACCACTTTGAGCTCTA & 63 & genotyping PCR, locus insertion \\
AAVS1_LUKI_CAG_R1 & GGCTATGAACTAATGACCCCG & 63 & genotyping PCR, locus insertion \\
GSP_1 & $\begin{array}{l}\text { ATTGGCGTTACTATGGGAACATA } \\
\text { CGTCATTAT }\end{array}$ & 60 & integration site analysis \\
GSP_2 & TGGGCGGGGGTCGTTGGGCGGT & 70 & integration site analysis \\
\hline
\end{tabular}

\subsection{Plasmid maps}

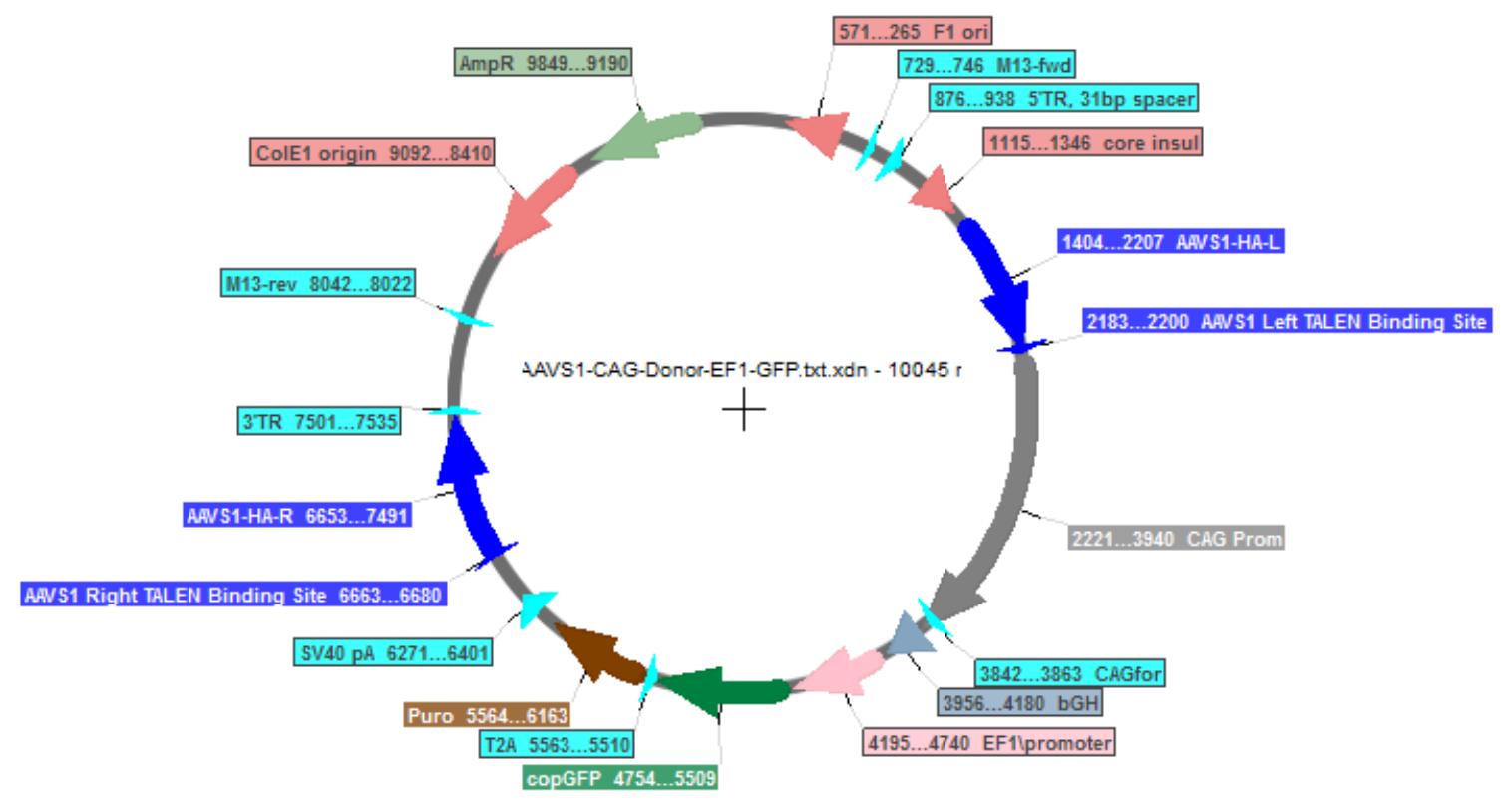

Figure 18 pAAVS1-CAG-GFP-puro, 10045 bp 


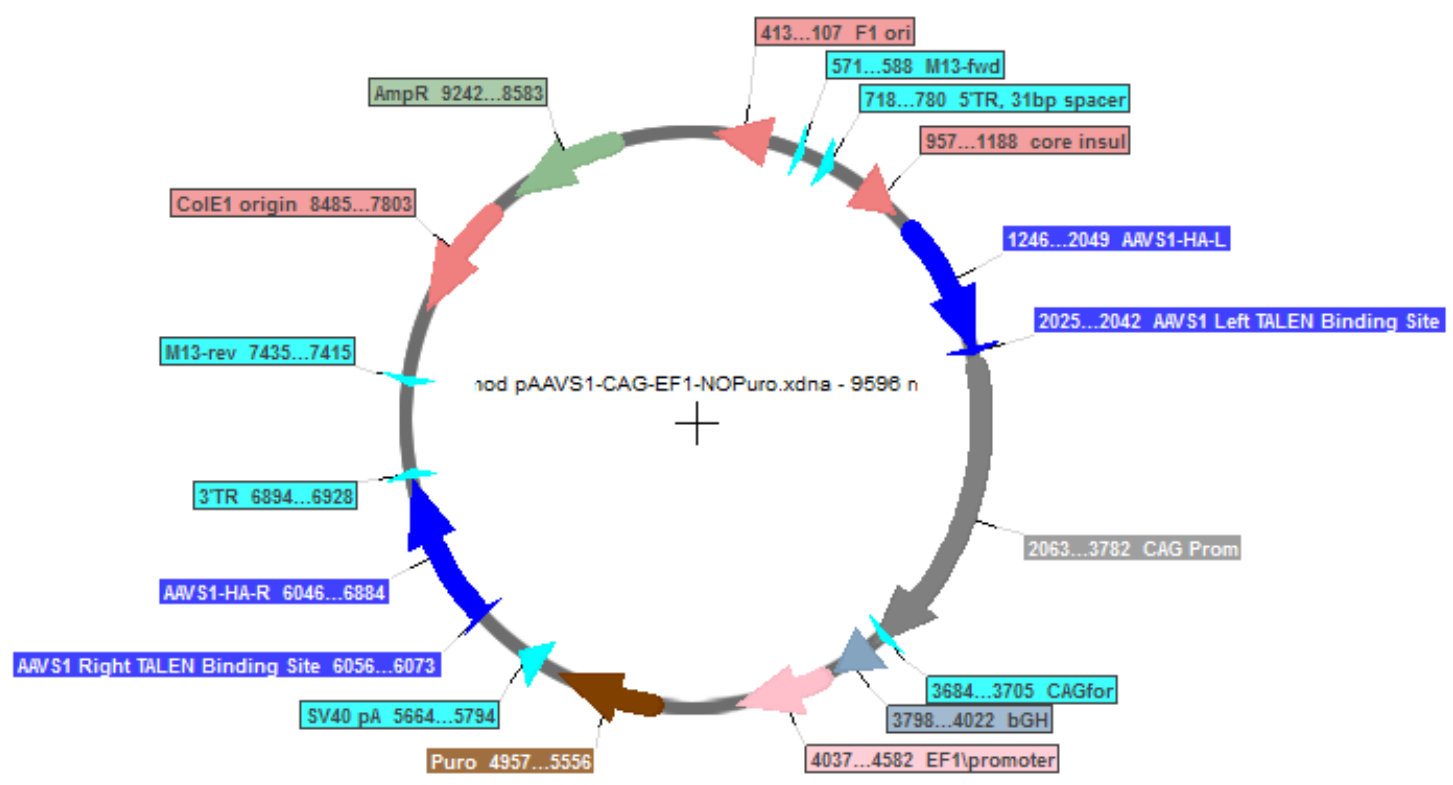

Figure 19 pAAVS1-CAG-EF1-frameshiftpuro, 9596 bp

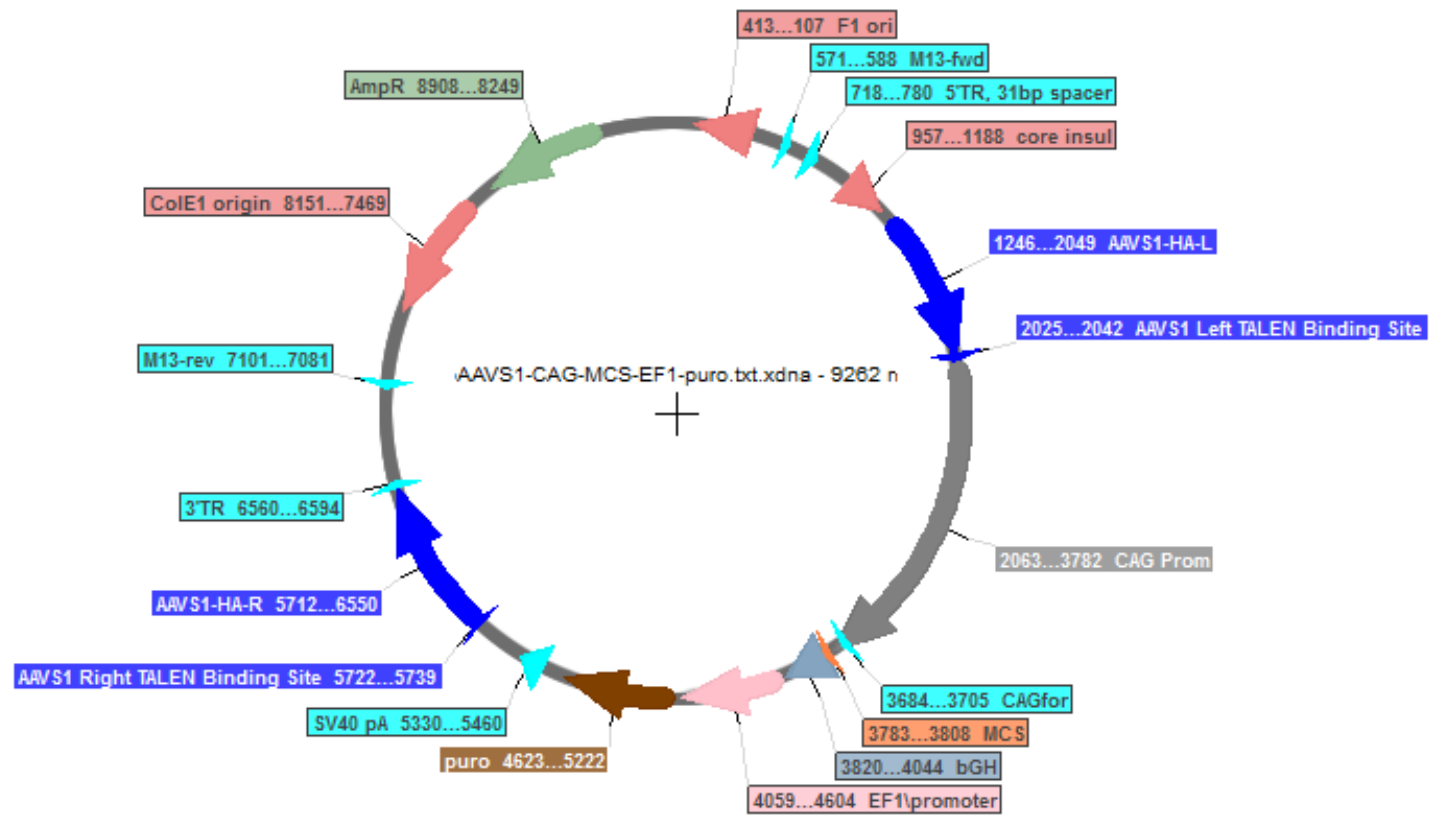

Figure 20 pAAVS1-CAG-MCS-EF1-puro, 9262 bp 


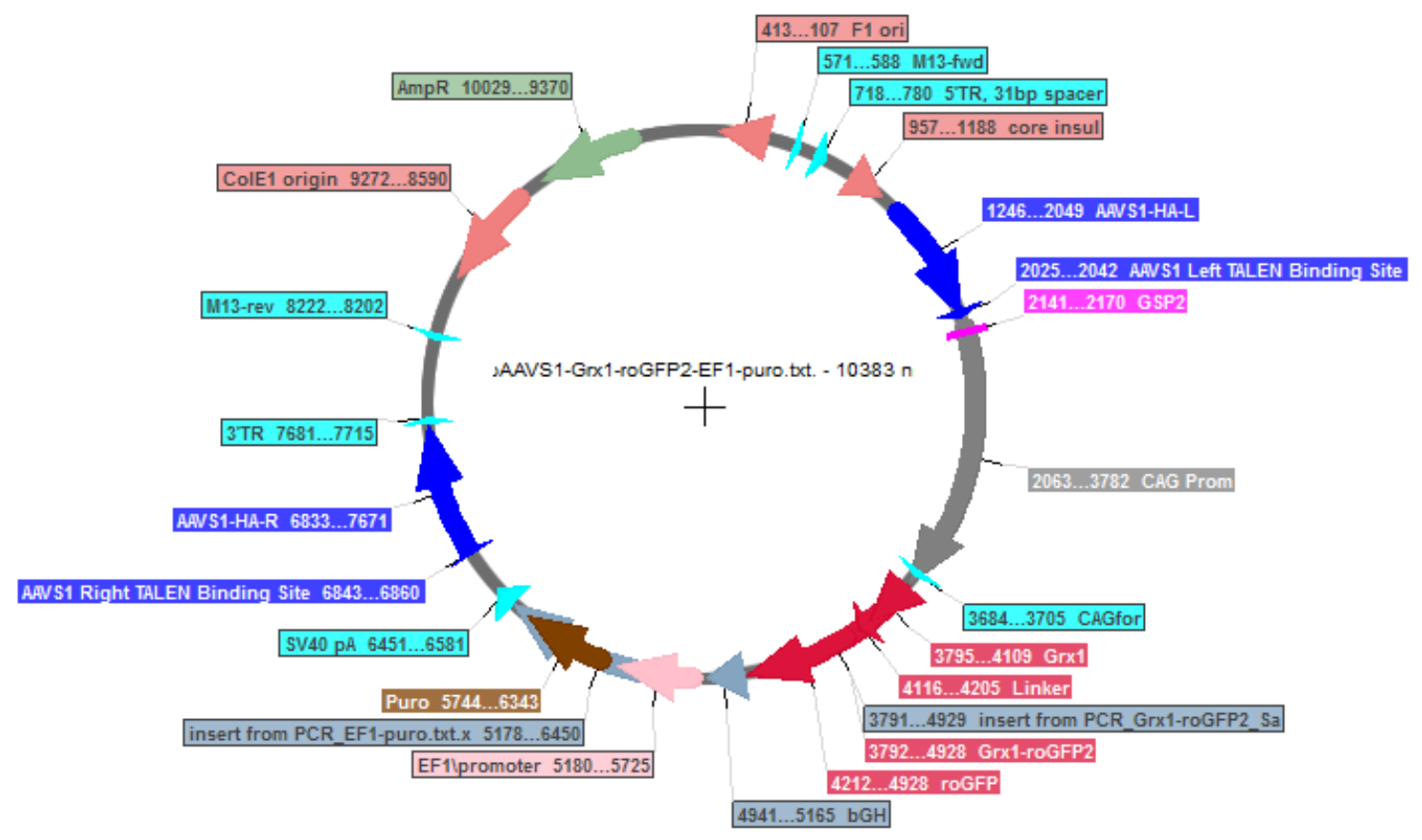

Figure 21 pAAVS1-Grx1-roGFP2, 10383 bp

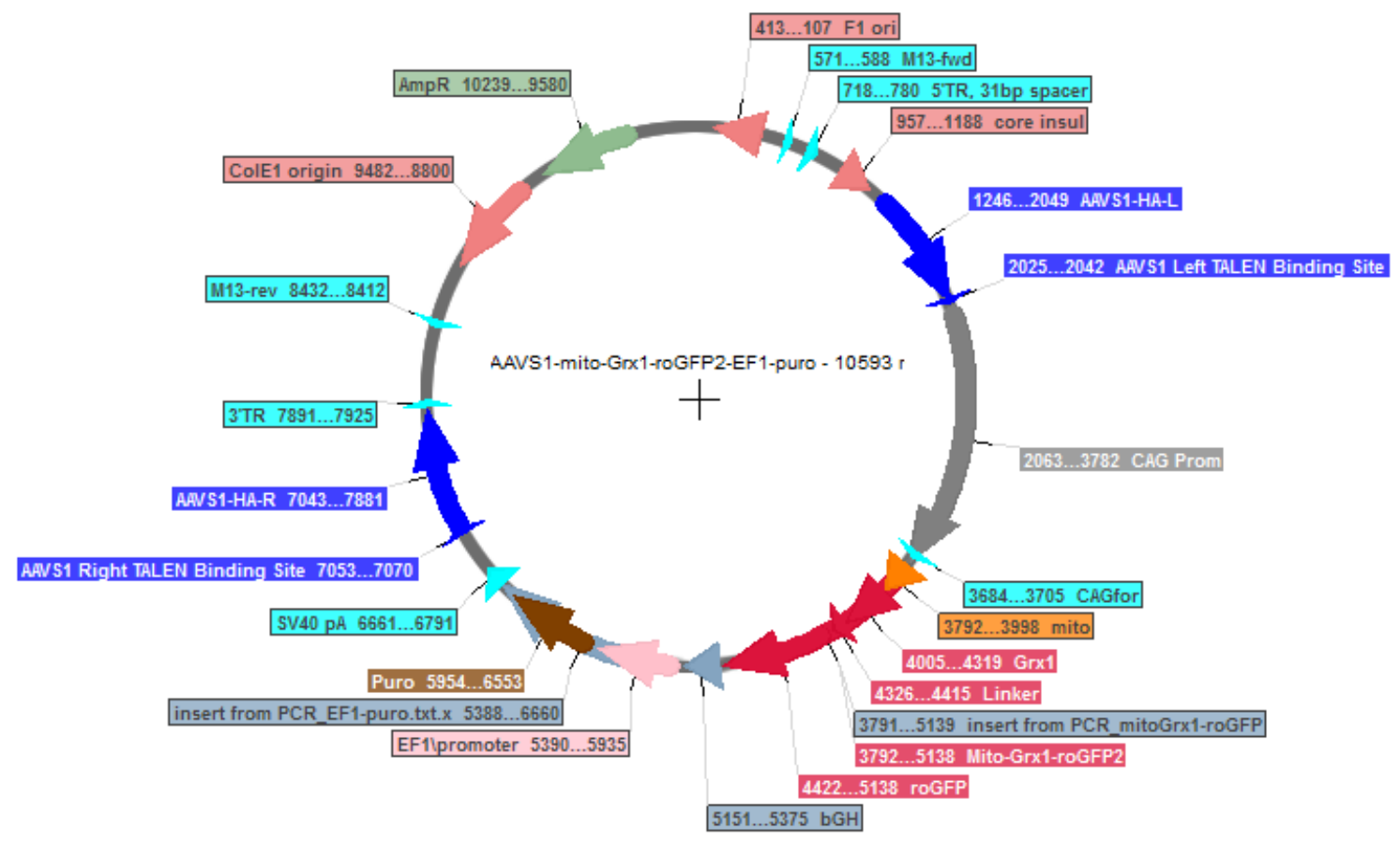

Figure 22 pAAVS1-mito-Grx1-roGFP2, 10596 bp 


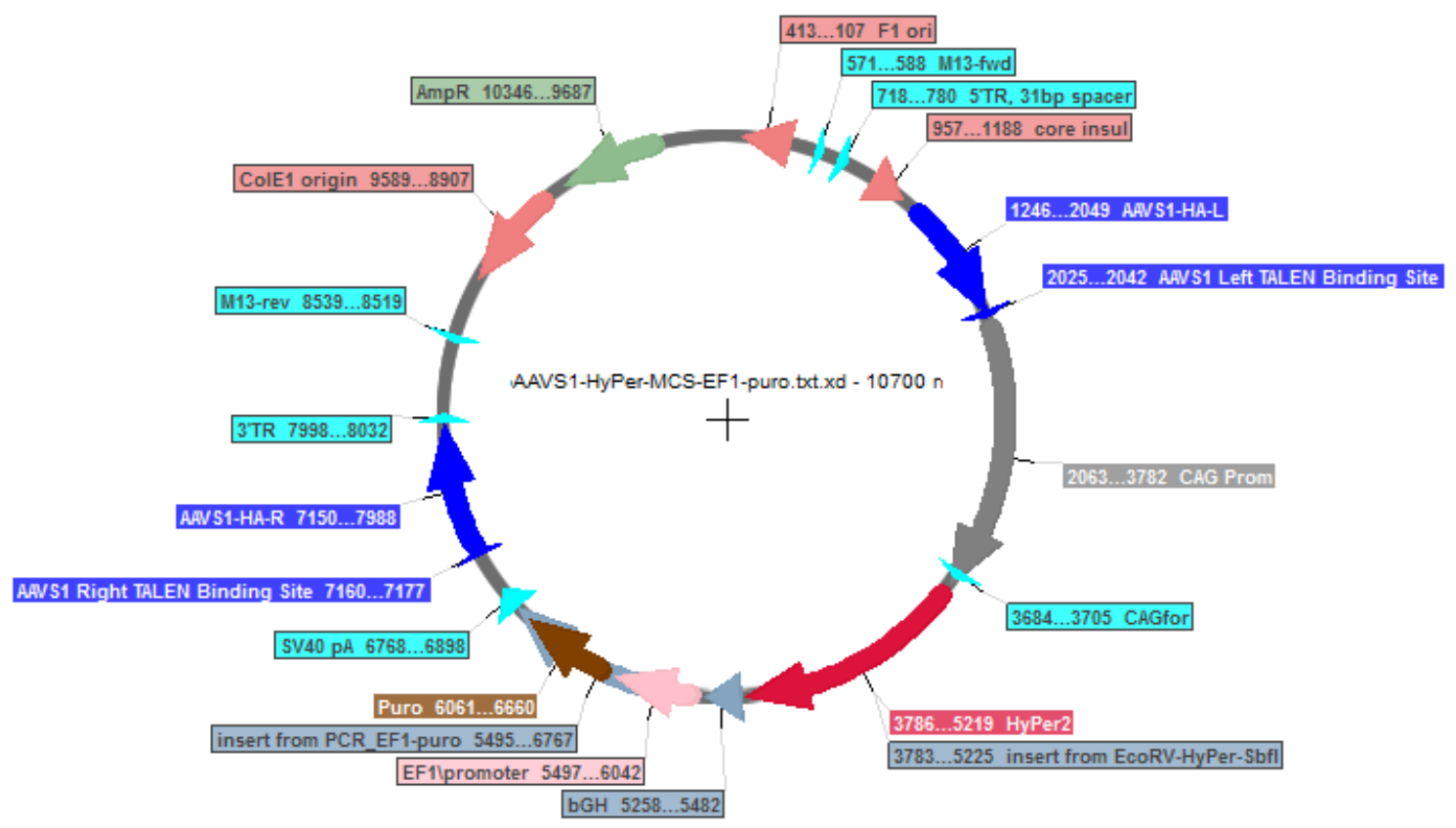

Figure 23 pAAVS1-HyPer, 10700 bp

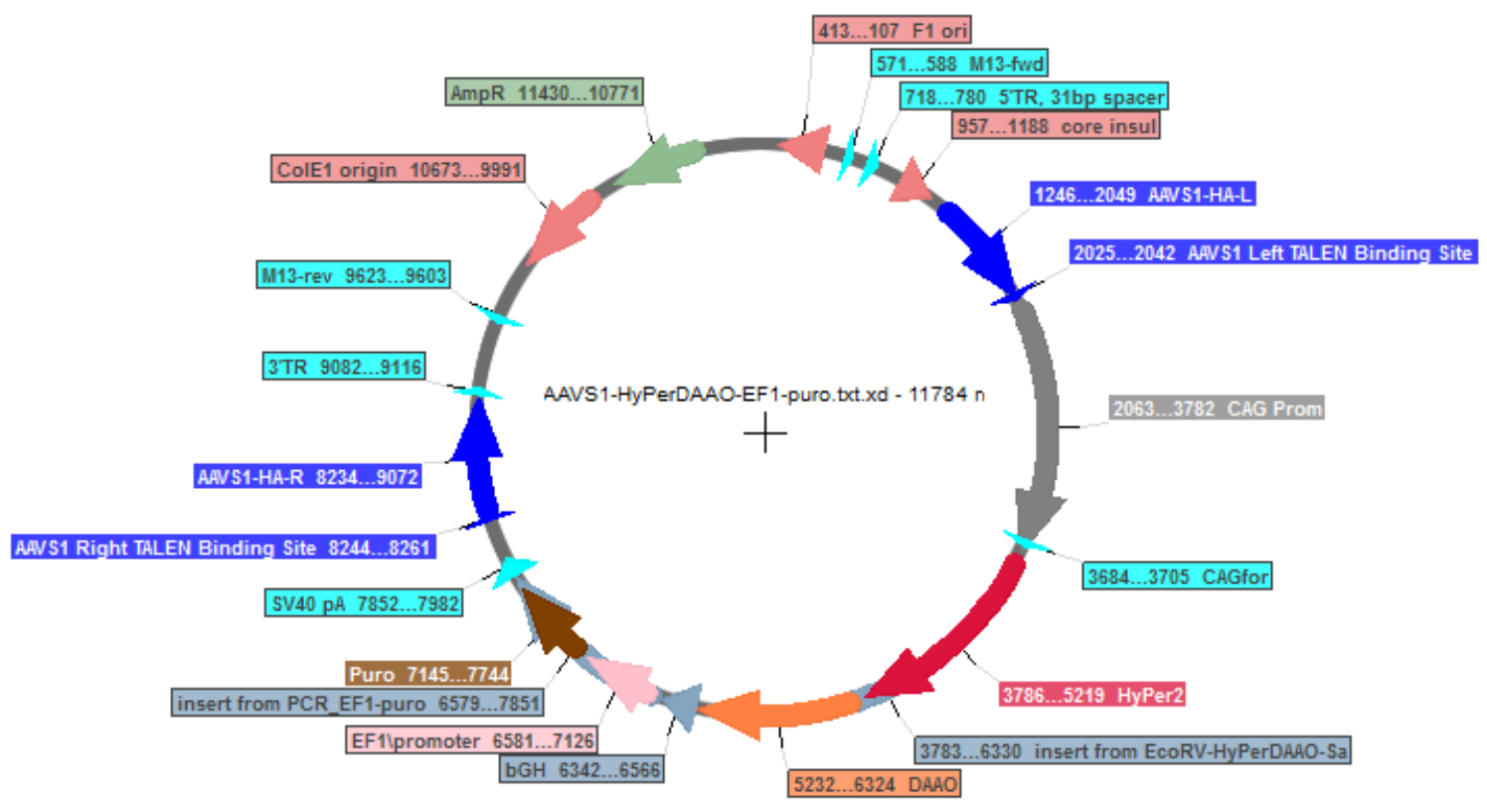

Figure 24 pAAVS1-HyPerDAO, 11784 bp 


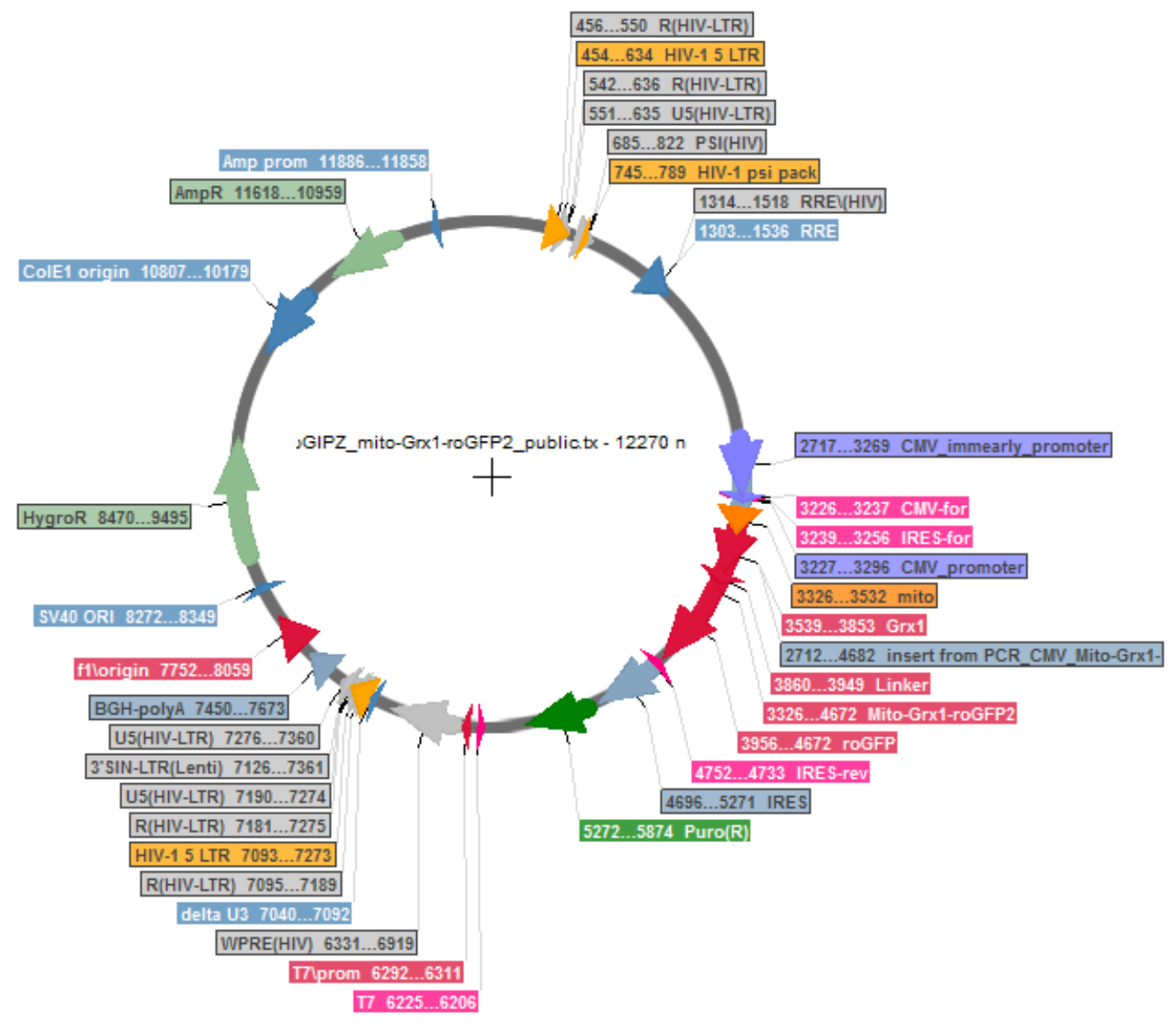

Figure 25 pGIPZ-mito-Grx1-roGFP2, 12270 bp 